\title{
High Trade Costs and Their Consequences: An Estimated Dynamic Model of African Agricultural Storage and Trade
}

\author{
Obie Porteous
}

\section{Online Appendix}

\section{A1 Data: Market Selection}

Table A1, which begins on the next page, includes two lists of markets by country and town population (in thousands). Population data is from the most recent available national censuses as reported in various online databases (e.g. citypopulation.de) and should be taken as approximate as census years vary by country. The "ideal" list starts with the 178 towns with a population of at least 100,000 that are at least 200 kilometers apart ${ }^{1}$ (plain font). When two towns of over 100,000 population are closer than 200 kilometers the larger is chosen. An additional 85 towns (italics) on this list are either located at important transport hubs (road junctions or ports) or are additional major towns in countries with high initial population-to-market ratios. The "actual" list is my final network of 230 markets. This includes 218 of the 263 markets on my ideal list for which I was able to obtain price data (plain font) as well as an additional 12 markets with price data which are located close to 12 of the missing markets and which I therefore use as substitutes (italics).

Table A2, which follows table A1, shows the population-to-market ratios by country for the two sets of markets. In the ideal list of markets, only Nigeria and Ethiopia - the two most populous countries - have population-to-market ratios above 4 million. In the final network, the three countries with more than two missing markets (Angola, Cameroon, and Uganda) are the only ones besides Nigeria and Ethiopia that are significantly above this threshold.

\footnotetext{
${ }^{1}$ Note that Johannesburg is the only town included in South Africa due to its special treatment in my model.
} 
Table A1: List of Markets by Country and Town Population (1/6)

\begin{tabular}{|c|c|c|c|c|}
\hline Country & Ideal & Population & Actual & Population \\
\hline \multirow[t]{9}{*}{ Angola } & Luanda & 2584 & Luanda & 2584 \\
\hline & Cabinda & 378 & & \\
\hline & Huambo & 333 & & \\
\hline & Lubango & 251 & & \\
\hline & Malanje & 157 & & \\
\hline & Lobito & 145 & & \\
\hline & Uige & 116 & & \\
\hline & Luena & 85 & & \\
\hline & Saurimo & 78 & & \\
\hline \multirow[t]{4}{*}{ Benin } & Cotonou & 818 & Cotonou & 818 \\
\hline & Parakou & 227 & Parakou & 227 \\
\hline & Kandi & 150 & Malanville & 36 \\
\hline & Natitingou & 120 & Natitingou & 120 \\
\hline \multirow{2}{*}{ Botswana } & Gaborone & 186 & Gaborone & 186 \\
\hline & Francistown & 83 & & \\
\hline \multirow[t]{5}{*}{ Burkina Faso } & Ouagadougou & 1182 & Ouagadougou & 1182 \\
\hline & Bobo Dioulasso & 436 & Bobo Dioulasso & 436 \\
\hline & Ouahigouya & 71 & & \\
\hline & Fada Ngourma & 41 & Fada Ngourma & 41 \\
\hline & Dedougou & 38 & Dedougou & 38 \\
\hline \multirow[t]{3}{*}{ Burundi } & Bujumbura & 340 & Bujumbura & 340 \\
\hline & Gitega & 47 & Gitega & 47 \\
\hline & Muyinga & 45 & Muyinga & 45 \\
\hline \multirow[t]{8}{*}{ Cameroon } & Douala & 1907 & Douala & 1907 \\
\hline & Yaounde & 1818 & Yaounde & 1818 \\
\hline & Bamenda & 270 & Bamenda & 270 \\
\hline & Garoua & 236 & Garoua & 236 \\
\hline & Maroua & 201 & & \\
\hline & Ngaoundere & 153 & & \\
\hline & Kousseri & 89 & & \\
\hline & Bertoua & 88 & & \\
\hline \multirow[t]{5}{*}{ Central African Republic } & Bangui & 623 & Bangui & 623 \\
\hline & Berberati & 77 & & \\
\hline & Bambari & 41 & Bambari & 41 \\
\hline & Bouar & 40 & & \\
\hline & Bangassou & 32 & Bangassou & 32 \\
\hline \multirow{4}{*}{ Chad } & Ndjamena & 818 & Ndjamena & 818 \\
\hline & Moundou & 141 & Moundou & 141 \\
\hline & Sarh & 119 & Sarh & 119 \\
\hline & Abeche & 77 & Abeche & 77 \\
\hline \multirow[t]{3}{*}{ Congo } & Brazzaville & 1373 & Brazzaville & 1373 \\
\hline & Pointe-Noire & 715 & Pointe-Noire & 715 \\
\hline & Impfondo & 34 & Impfondo & 34 \\
\hline
\end{tabular}


Table A1: List of Markets by Country and Town Population (2/6)

\begin{tabular}{|c|c|c|c|c|}
\hline Country & Ideal & Population & Actual & Population \\
\hline \multirow[t]{7}{*}{ Côte d'Ivoire } & Abdijan & 3677 & Abdijan & 3677 \\
\hline & Bouake & 567 & Bouake & 567 \\
\hline & Daloa & 216 & Daloa & 216 \\
\hline & San Pedro & 197 & & \\
\hline & Korhogo & 167 & Odienne & 43 \\
\hline & Man & 139 & Man & 139 \\
\hline & Abengourou & 71 & Abengourou & 71 \\
\hline \multirow[t]{22}{*}{ D.R. Congo } & Kinshasa & 8901 & Kinshasa & 8901 \\
\hline & Lubumbashi & 1630 & Lubumbashi & 1630 \\
\hline & Mbuji-Mayi & 1559 & Mbuji-Mayi & 1559 \\
\hline & Kisangani & 868 & Kisangani & 868 \\
\hline & Bukavu & 707 & Bukavu & 707 \\
\hline & Tshikapa & 524 & Tshikapa & 524 \\
\hline & Kolwezi & 451 & Kolwezi & 451 \\
\hline & Goma & 377 & Goma & 377 \\
\hline & Kikwit & 370 & Kikwit & 370 \\
\hline & Bunia & 327 & Bunia & 327 \\
\hline & Mbandaka & 324 & Mbandaka & 324 \\
\hline & Matadi & 291 & Matadi & 291 \\
\hline & Butembo & 204 & Butembo & 204 \\
\hline & Isiro & 175 & Isiro & 175 \\
\hline & Kindu & 164 & Kindu & 164 \\
\hline & Kamina & 144 & Kamina & 144 \\
\hline & Bandundu & 137 & Bandundu & 137 \\
\hline & Gemena & 133 & Zongo & 33 \\
\hline & Bumba & 103 & Gbadolite & 48 \\
\hline & Kananga & 967 & Kananga & 967 \\
\hline & Uvira & 337 & Uvira & 337 \\
\hline & Kalemie & 92 & Kalemie & 92 \\
\hline Djibouti & Djibouti & 624 & Djibouti & 624 \\
\hline \multirow[t]{3}{*}{ Eritrea } & Asmara & 650 & Asmara & 650 \\
\hline & Teseney & 65 & & \\
\hline & Massawa & 37 & Massawa & 37 \\
\hline \multirow[t]{15}{*}{ Ethiopia } & Addis Ababa & 3041 & Addis Ababa & 3041 \\
\hline & Dire Dawa & 274 & Dire Dawa & 274 \\
\hline & Mekele & 272 & Mekele & 272 \\
\hline & Gondar & 254 & Gondar & 254 \\
\hline & Awasa & 213 & Awasa & 213 \\
\hline & Jimma & 149 & Jimma & 149 \\
\hline & Dessie & 148 & Dessie & 148 \\
\hline & Bahir Dar & 191 & Bahir Dar & 191 \\
\hline & Jijiga & 147 & Jijiga & 147 \\
\hline & Arba Minch & 96 & & \\
\hline & Nekemte & 89 & Nekemte & 89 \\
\hline & Gode & 68 & Gode & 68 \\
\hline & $A d w a$ & 41 & & \\
\hline & Gambela & 39 & Gambela & 39 \\
\hline & Moyale & 34 & Yabelo & 18 \\
\hline
\end{tabular}


Table A1: List of Markets by Country and Town Population (3/6)

\begin{tabular}{|c|c|c|c|c|}
\hline Country & Ideal & Population & Actual & Population \\
\hline \multirow[t]{2}{*}{ Gabon } & Libreville & 591 & Libreville & 591 \\
\hline & Port Gentil & 112 & & \\
\hline Gambia & Banjul & 524 & Banjul & 524 \\
\hline \multirow[t]{7}{*}{ Ghana } & Accra & 2070 & Accra & 2070 \\
\hline & Kumasi & 2035 & Kumasi & 2035 \\
\hline & Tamale & 371 & Tamale & 371 \\
\hline & Sekondi Takoradi & 539 & Sekondi Takoradi & 539 \\
\hline & Ho & 105 & Но & 105 \\
\hline & $W a$ & 71 & Wa & 71 \\
\hline & Bolgatanga & 66 & Bolgatanga & 66 \\
\hline \multirow[t]{6}{*}{ Guinea } & Conakry & 1400 & Conakry & 1400 \\
\hline & Nzerekore & 178 & Nzerekore & 178 \\
\hline & Boke & 147 & & \\
\hline & Kankan & 142 & Kankan & 142 \\
\hline & Gueckedou & 96 & & \\
\hline & Mamou & 60 & Labe & 59 \\
\hline Guinea Bissau & Bissau & 388 & Bissau & 388 \\
\hline \multirow[t]{11}{*}{ Kenya } & Nairobi & 3138 & Nairobi & 3138 \\
\hline & Mombasa & 939 & Mombasa & 939 \\
\hline & Kisumu & 388 & Kisumu & 388 \\
\hline & Garissa & 116 & Garissa & 116 \\
\hline & Nakuru & 308 & Nakuru & 308 \\
\hline & Eldoret & 289 & Eldoret & 289 \\
\hline & Mandera & 88 & Mandera & 88 \\
\hline & Wajir & 82 & Wajir & 82 \\
\hline & Lodwar & 48 & Lodwar & 48 \\
\hline & Isiolo & 46 & & \\
\hline & Moyale & 38 & Moyale & 38 \\
\hline Lesotho & Maseru & 218 & Maseru & 218 \\
\hline \multirow[t]{2}{*}{ Liberia } & Monrovia & 1022 & Monrovia & 1022 \\
\hline & Gbarnga & 57 & Gbarnga & 57 \\
\hline \multirow[t]{5}{*}{ Malawi } & Lilongwe & 647 & Lilongwe & 647 \\
\hline & Blantyre & 585 & Blantyre & 585 \\
\hline & Mzuzu & 175 & Mzuzu & 175 \\
\hline & Mangochi & 40 & Mangochi & 40 \\
\hline & Karonga & 34 & Karonga & 34 \\
\hline \multirow[t]{6}{*}{ Mali } & Bamako & 1809 & Bamako & 1809 \\
\hline & Sikasso & 226 & Sikasso & 226 \\
\hline & Segou & 131 & Segou & 131 \\
\hline & Kayes & 127 & Kayes & 127 \\
\hline & Mopti & 114 & Mopti & 114 \\
\hline & Gao & 87 & Gao & 87 \\
\hline
\end{tabular}


Table A1: List of Markets by Country and Town Population (4/6)

\begin{tabular}{|c|c|c|c|c|}
\hline Country & Ideal & Population & Actual & Population \\
\hline \multirow[t]{4}{*}{ Mauritania } & Nouakchott & 719 & Nouakchott & 719 \\
\hline & Nouadhibou & 90 & & \\
\hline & Adel Bagrou & 58 & Adel Bagrou & 58 \\
\hline & Kiffa & 40 & Tintane & 22 \\
\hline \multirow[t]{13}{*}{ Mozambique } & Maputo & 1766 & Maputo & 1766 \\
\hline & Beira & 546 & Beira & 546 \\
\hline & Nampula & 478 & Nampula & 478 \\
\hline & Chimoio & 239 & Chimoio & 239 \\
\hline & Quelimane & 193 & Quelimane & 193 \\
\hline & Tete & 156 & Tete & 156 \\
\hline & Lichinga & 142 & Lichinga & 142 \\
\hline & Pemba & 141 & Pemba & 141 \\
\hline & Gurue & 117 & Cuamba & 95 \\
\hline & Xai Xai & 116 & Xai Xai & 116 \\
\hline & Maxixe & 106 & Maxixe & 106 \\
\hline & Nacala & 208 & Nacala & 208 \\
\hline & Milange & 30 & Milange & 30 \\
\hline \multirow{4}{*}{ Namibia } & Windhoek & 268 & Windhoek & 268 \\
\hline & Rundu & 58 & Katima Mulilo & 28 \\
\hline & Walvis Bay & 52 & Swakopmund & 44 \\
\hline & Oshakati & 37 & Oshakati & 37 \\
\hline \multirow[t]{7}{*}{ Niger } & Niamey & 1303 & Niamey & 1303 \\
\hline & Zinder & 275 & Zinder & 275 \\
\hline & Maradi & 206 & Maradi & 206 \\
\hline & Agadez & 124 & Agadez & 124 \\
\hline & Tahoua & 123 & Tahoua & 123 \\
\hline & Arlit & 112 & Arlit & 112 \\
\hline & Diffa & 48 & Diffa & 48 \\
\hline \multirow[t]{20}{*}{ Nigeria } & Lagos & 8029 & Lagos & 8029 \\
\hline & Kano & 3249 & Kano & 3249 \\
\hline & Kaduna & 1459 & Kaduna & 1459 \\
\hline & Port Harcourt & 1054 & Port Harcourt & 1054 \\
\hline & Benin City & 1052 & Benin City & 1052 \\
\hline & Maiduguri & 972 & Maiduguri & 972 \\
\hline & Ilorin & 756 & Ilorin & 756 \\
\hline & Jos & 742 & Jos & 742 \\
\hline & Enugu & 593 & Enugu & 593 \\
\hline & Sokoto & 501 & Sokoto & 501 \\
\hline & Okene & 445 & Lokoja & 90 \\
\hline & Calabar & 431 & Calabar & 431 \\
\hline & Makurdi & 249 & Makurdi & 249 \\
\hline & Gombe & 231 & Gombe & 231 \\
\hline & Yola & 218 & Yola & 218 \\
\hline & Abuja & 160 & Abuja & 160 \\
\hline & Gashua & 110 & & \\
\hline & Ibadan & 3078 & Ibadan & 3078 \\
\hline & Katsina & 387 & Katsina & 387 \\
\hline & Akure & 370 & Akure & 370 \\
\hline
\end{tabular}


Table A1: List of Markets by Country and Town Population (5/6)

\begin{tabular}{|c|c|c|c|c|}
\hline Country & Ideal & Population & Actual & Population \\
\hline \multirow[t]{3}{*}{ Rwanda } & Kigali & 745 & Kigali & 745 \\
\hline & Butare & 90 & Butare & 90 \\
\hline & Gisenyi & 84 & Gisenyi & 84 \\
\hline \multirow[t]{6}{*}{ Senegal } & Dakar & 1999 & Dakar & 1999 \\
\hline & Ziguinchor & 162 & Ziguinchor & 162 \\
\hline & Saint Louis & 131 & Saint Louis & 131 \\
\hline & Touba & 428 & Touba & 428 \\
\hline & Kaolack & 174 & Kaolack & 174 \\
\hline & Tambacounda & 75 & Tambacounda & 75 \\
\hline \multirow[t]{3}{*}{ Sierra Leone } & Freetown & 773 & Freetown & 773 \\
\hline & Bo & 150 & Bo & 150 \\
\hline & Kabala & 14 & Kabala & 14 \\
\hline \multirow[t]{9}{*}{ Somalia } & Mogadishu & 1353 & Mogadishu & 1353 \\
\hline & Hargeisa & 1200 & Hargeisa & 1200 \\
\hline & Bosaso & 700 & Bosaso & 700 \\
\hline & Galkayo & 545 & Galkayo & 545 \\
\hline & Kismayo & 183 & Kismayo & 183 \\
\hline & Baidoa & 158 & Baidoa & 158 \\
\hline & Berbera & 233 & & \\
\hline & Beledweyne & 67 & Beledweyne & 67 \\
\hline & Garoowe & 57 & Garoowe & 57 \\
\hline South Africa & Johannesburg & 957 & Johannesburg & 957 \\
\hline \multirow[t]{6}{*}{ South Sudan } & Juba & 372 & Juba & 372 \\
\hline & Wau & 151 & Wau & 151 \\
\hline & Malakal & 139 & Malakal & 139 \\
\hline & Yambio & 40 & & \\
\hline & Rumbek & 32 & Rumbek & 32 \\
\hline & Bor & 27 & Bor & 27 \\
\hline \multirow[t]{12}{*}{ Sudan } & Khartoum & 4273 & Khartoum & 4273 \\
\hline & Nyala & 493 & Nyala & 493 \\
\hline & Port Sudan & 395 & Port Sudan & 395 \\
\hline & El Obeid & 345 & El Obeid & 345 \\
\hline & Kassala & 299 & Kassala & 299 \\
\hline & $\mathrm{Al}$ Qadarif & 269 & Al Qadarif & 269 \\
\hline & Al Fashir & 218 & Al Fashir & 218 \\
\hline & Kostil & 213 & Kostil & 213 \\
\hline & Ad Damazin & 137 & Ad Damazin & 137 \\
\hline & El Geneina & 134 & El Geneina & 134 \\
\hline & Atbarah & 112 & & \\
\hline & Kadugli & 67 & Kadugli & 67 \\
\hline Swaziland & Mbabane & 95 & Mbabane & 95 \\
\hline
\end{tabular}


Table A1: List of Markets by Country and Town Population (6/6)

\begin{tabular}{|c|c|c|c|c|}
\hline Country & Ideal & Population & Actual & Population \\
\hline \multirow[t]{16}{*}{ Tanzania } & Dar es Salaam & 4365 & Dar es Salaam & 4365 \\
\hline & Mwanza & 707 & Mwanza & 707 \\
\hline & Arusha & 416 & Arusha & 416 \\
\hline & Dodoma & 411 & Dodoma & 411 \\
\hline & Mbeya & 385 & Mbeya & 385 \\
\hline & Tanga & 273 & Tanga & 273 \\
\hline & Tabora & 227 & Tabora & 227 \\
\hline & Kigoma & 215 & Kigoma & 215 \\
\hline & Sumbawanga & 210 & Sumbawanga & 210 \\
\hline & Songea & 203 & Songea & 203 \\
\hline & Musoma & 178 & Musoma & 178 \\
\hline & Iringa & 151 & Iringa & 151 \\
\hline & Singida & 150 & Singida & 150 \\
\hline & Bukoba & 129 & Bukoba & 129 \\
\hline & Mtwara & 108 & Mtwara & 108 \\
\hline & Mpanda & 102 & & \\
\hline \multirow[t]{2}{*}{ Togo } & Lome & 729 & Lome & 729 \\
\hline & Sokode & 118 & Kara & 104 \\
\hline \multirow[t]{10}{*}{ Uganda } & Kampala & 1660 & Kampala & 1660 \\
\hline & Gulu & 154 & Gulu & 154 \\
\hline & Lira & 108 & Lira & 108 \\
\hline & Mbale & 92 & & \\
\hline & Jinja & 90 & Jinja & 90 \\
\hline & Mbarara & 84 & Mbarara & 84 \\
\hline & Kasese & 74 & & \\
\hline & Masaka & 74 & & \\
\hline & Arua & 59 & Arua & 59 \\
\hline & Masindi & 45 & Masindi & 45 \\
\hline \multirow[t]{8}{*}{ Zambia } & Lusaka & 2147 & Lusaka & 2147 \\
\hline & Kitwe & 410 & Kitwe & 410 \\
\hline & Chipata & 117 & Chipata & 117 \\
\hline & Livingstone & 113 & Livingstone & 113 \\
\hline & Kasama & 102 & Kasama & 102 \\
\hline & Kabwe & 193 & Kabwe & 193 \\
\hline & Solwezi & 91 & Solwezi & 91 \\
\hline & Mongu & 52 & Mongu & 52 \\
\hline \multirow[t]{5}{*}{ Zimbabwe } & Harare & 1607 & Harare & 1607 \\
\hline & Bulawayo & 713 & Bulawayo & 713 \\
\hline & Mutare & 194 & Mutare & 194 \\
\hline & Masvingo & 81 & Masvingo & 81 \\
\hline & Hwange & 37 & Hwange & 37 \\
\hline
\end{tabular}


Table A2: Population (2010, Millions) per Market by Country

\begin{tabular}{|c|c|c|c|c|c|}
\hline Country & Population & Ideal & Popn/Mkt & Actual & Popn/Mkt \\
\hline Angola & 19.55 & 9 & 2.17 & 1 & 19.55 \\
\hline Benin & 9.51 & 4 & 2.38 & 4 & 2.38 \\
\hline Botswana & 1.97 & 2 & 0.98 & 1 & 1.97 \\
\hline Burkina Faso & 15.54 & 5 & 3.11 & 4 & 3.89 \\
\hline Burundi & 9.23 & 3 & 3.08 & 3 & 3.08 \\
\hline Cameroon & 20.62 & 8 & 2.58 & 4 & 5.16 \\
\hline Central African Republic & 4.35 & 5 & 0.87 & 3 & 1.45 \\
\hline Chad & 11.72 & 4 & 2.93 & 4 & 2.93 \\
\hline Congo & 4.11 & 3 & 1.37 & 3 & 1.37 \\
\hline Côte d'Ivoire & 18.98 & 7 & 2.71 & 6 & 3.16 \\
\hline D.R. Congo & 62.19 & 22 & 2.83 & 22 & 2.83 \\
\hline Djibouti & 0.83 & 1 & 0.83 & 1 & 0.83 \\
\hline Eritrea & 5.74 & 3 & 1.91 & 2 & 2.87 \\
\hline Ethiopia & 87.10 & 15 & 5.81 & 13 & 6.70 \\
\hline Gabon & 1.56 & 2 & 0.78 & 1 & 1.56 \\
\hline Gambia & 1.68 & 1 & 1.68 & 1 & 1.68 \\
\hline Ghana & 24.26 & 7 & 3.47 & 7 & 3.47 \\
\hline Guinea & 10.88 & 6 & 1.81 & 4 & 2.72 \\
\hline Guinea Bissau & 1.59 & 1 & 1.59 & 1 & 1.59 \\
\hline Kenya & 40.91 & 11 & 3.72 & 10 & 4.09 \\
\hline Lesotho & 2.01 & 1 & 2.01 & 1 & 2.01 \\
\hline Liberia & 3.96 & 2 & 1.98 & 2 & 1.98 \\
\hline Malawi & 15.01 & 5 & 3.00 & 5 & 3.00 \\
\hline Mali & 13.99 & 6 & 2.33 & 6 & 2.33 \\
\hline Mauritania & 3.61 & 4 & 0.90 & 3 & 1.20 \\
\hline Mozambique & 23.97 & 13 & 1.84 & 13 & 1.84 \\
\hline Namibia & 2.18 & 4 & 0.54 & 4 & 0.54 \\
\hline Niger & 15.89 & 7 & 2.27 & 7 & 2.27 \\
\hline Nigeria & 159.71 & 20 & 7.99 & 19 & 8.41 \\
\hline Rwanda & 10.84 & 3 & 3.61 & 3 & 3.61 \\
\hline Senegal & 12.95 & 6 & 2.16 & 6 & 2.16 \\
\hline Sierra Leone & 5.75 & 3 & 1.92 & 3 & 1.92 \\
\hline Somalia & 9.64 & 9 & 1.07 & 8 & 1.20 \\
\hline South Africa & NA & 1 & NA & 1 & NA \\
\hline South Sudan & 9.94 & 6 & 1.66 & 5 & 1.99 \\
\hline Sudan & 35.65 & 12 & 2.97 & 11 & 3.24 \\
\hline Swaziland & 1.19 & 1 & 1.19 & 1 & 1.19 \\
\hline Tanzania & 44.97 & 16 & 2.81 & 15 & 3.00 \\
\hline Togo & 6.31 & 2 & 3.15 & 2 & 3.15 \\
\hline Uganda & 33.99 & 10 & 3.40 & 7 & 4.86 \\
\hline Zambia & 13.22 & 8 & 1.65 & 8 & 1.65 \\
\hline Zimbabwe & 13.08 & 5 & 2.62 & 5 & 2.62 \\
\hline Total & 842.31 & 263 & 3.20 & 230 & 3.66 \\
\hline
\end{tabular}




\section{A2 Data: Grain Types and Data Sources}

Table A3, which begins on the next page, lists the seasonal regime and the grain types for each of the 230 markets in my final network. 112 markets fall into the Northern Hemisphere zone (N) with a single annual grain harvest in October, 70 markets fall into the Equatorial zone (E) with a larger grain harvest (two-thirds of the annual total) in July and a smaller grain harvest (one-third of the annual total) in December, and 48 markets fall into the Southern Hemisphere zone (S) with a single annual grain harvest in May.

To determine the grain types for each market, I first made a list for each country of all cereal grains constituting at least 5 percent of national cereal grain production. I excluded barley (Eritrea and Ethiopia) and fonio (Guinea) as they are relatively minor grains. I then searched for available price data for these grains and removed a few from the final list so as to have contiguous areas for each grain for my trade network. 86 percent of total cereal grain production in my countries of interest is covered by a grain price series in its associated market.

Table A4, which follows table A3, lists the data sources by country for my price data. Most series were obtained from secondary sources, particularly the online databases maintained by the World Food Programme's VAM unit and FAO's GIEWS project. However, table A3 also includes the primary sources from which these databases obtained their price data. Although the price data are often collected by different government ministries in different countries, the methodology is typically quite similar and the mandate usually falls into one of three categories: (i) agricultural market information systems (MIS) intended to provide information to farmers and traders on market prices in different locations; (ii) price monitoring by national statistics offices for the monthly consumer price index (CPI); or (iii) food security monitoring by agencies like the World Food Programme. 
Table A3: Seasonal Regime and Grain Types by Market (1/6)

\begin{tabular}{|c|c|c|c|c|c|c|c|c|}
\hline Country & Market & Season & Maize & Millet & Rice & Sorghum & Teff & Wheat \\
\hline Angola & Luanda & $\mathrm{E}$ & $\mathrm{X}$ & & & & & \\
\hline \multirow[t]{4}{*}{ Benin } & Cotonou & $\mathrm{N}$ & $\mathrm{X}$ & & $\mathrm{X}$ & & & \\
\hline & Malanville & $\mathrm{N}$ & $\mathrm{X}$ & & & $\mathrm{X}$ & & \\
\hline & Natitingou & $\mathrm{N}$ & $\mathrm{X}$ & & $\mathrm{X}$ & $\mathrm{X}$ & & \\
\hline & Parakou & $\mathrm{N}$ & $\mathrm{X}$ & & $\mathrm{X}$ & & & \\
\hline Botswana & Gaborone & $\mathrm{S}$ & $\mathrm{X}$ & & & & & \\
\hline \multirow[t]{4}{*}{ Burkina Faso } & Bobo Dioulasso & $\mathrm{N}$ & & $\mathrm{X}$ & & $\mathrm{X}$ & & \\
\hline & Dedougou & $\mathrm{N}$ & & $\mathrm{X}$ & & $\mathrm{X}$ & & \\
\hline & Fada Ngourma & $\mathrm{N}$ & & $\mathrm{X}$ & & $\mathrm{X}$ & & \\
\hline & Ouagadougou & $\mathrm{N}$ & & $\mathrm{X}$ & & $\mathrm{X}$ & & \\
\hline \multirow[t]{3}{*}{ Burundi } & Bujumbura & $\mathrm{E}$ & $\mathrm{X}$ & & & $\mathrm{X}$ & & \\
\hline & Gitega & $\mathrm{E}$ & $\mathrm{X}$ & & & $\mathrm{X}$ & & \\
\hline & Muyinga & $\mathrm{E}$ & $\mathrm{X}$ & & & $\mathrm{X}$ & & \\
\hline \multirow[t]{4}{*}{ Cameroon } & Bamenda & $\mathrm{N}$ & $\mathrm{X}$ & & $\mathrm{X}$ & & & \\
\hline & Douala & $\mathrm{N}$ & $\mathrm{X}$ & & $\mathrm{X}$ & & & \\
\hline & Garoua & $\mathrm{N}$ & $\mathrm{X}$ & & $\mathrm{X}$ & & & \\
\hline & Yaounde & $\mathrm{N}$ & $\mathrm{X}$ & & $\mathrm{X}$ & & & \\
\hline \multirow[t]{3}{*}{ Central African Republic } & Bambari & $\mathrm{E}$ & $\mathrm{X}$ & & $\mathrm{X}$ & & & \\
\hline & Bangassou & $\mathrm{E}$ & $\mathrm{X}$ & & $\mathrm{X}$ & & & \\
\hline & Bangui & $\mathrm{E}$ & $\mathrm{X}$ & & $\mathrm{X}$ & & & \\
\hline \multirow[t]{4}{*}{ Chad } & Abeche & $\mathrm{N}$ & & $\mathrm{X}$ & & $\mathrm{X}$ & & \\
\hline & Moundou & $\mathrm{N}$ & & $\mathrm{X}$ & & $\mathrm{X}$ & & \\
\hline & Ndjamena & $\mathrm{N}$ & $\mathrm{X}$ & $\mathrm{X}$ & $\mathrm{X}$ & $\mathrm{X}$ & & \\
\hline & Sarh & $\mathrm{N}$ & & $\mathrm{X}$ & & $\mathrm{X}$ & & \\
\hline \multirow[t]{3}{*}{ Congo } & Brazzaville & $\mathrm{E}$ & & & $\mathrm{X}$ & & & \\
\hline & Impfondo & $\mathrm{E}$ & & & $\mathrm{X}$ & & & \\
\hline & Pointe Noire & $\mathrm{E}$ & & & $\mathrm{X}$ & & & \\
\hline \multirow[t]{6}{*}{ Côte d'Ivoire } & Abengourou & $\mathrm{N}$ & $\mathrm{X}$ & & $\mathrm{X}$ & & & \\
\hline & Abidjan & $\mathrm{N}$ & $\mathrm{X}$ & & $\mathrm{X}$ & & & \\
\hline & Bouake & $\mathrm{N}$ & $\mathrm{X}$ & & $\mathrm{X}$ & & & \\
\hline & Daloa & $\mathrm{N}$ & $\mathrm{X}$ & & $\mathrm{X}$ & & & \\
\hline & Man & $\mathrm{N}$ & $\mathrm{X}$ & & $\mathrm{X}$ & & & \\
\hline & Odienne & $\mathrm{N}$ & $\mathrm{X}$ & & $\mathrm{X}$ & & & \\
\hline
\end{tabular}


Table A3: Seasonal Regime and Grain Types by Market (2/6)

\begin{tabular}{|c|c|c|c|c|c|c|c|c|}
\hline Country & Market & Season & Maize & Millet & Rice & Sorghum & Teff & Wheat \\
\hline \multirow{22}{*}{ D.R. Congo } & Bandundu & $\mathrm{E}$ & $\mathrm{X}$ & & $\mathrm{X}$ & & & \\
\hline & Bukavu & $\mathrm{E}$ & $\mathrm{X}$ & & $\mathrm{X}$ & & & \\
\hline & Bunia & $\mathrm{E}$ & $\mathrm{X}$ & & $\mathrm{X}$ & & & \\
\hline & Butembo & $\mathrm{E}$ & $\mathrm{X}$ & & $\mathrm{X}$ & & & \\
\hline & Gbadolite & $\mathrm{E}$ & $\mathrm{X}$ & & $\mathrm{X}$ & & & \\
\hline & Goma & $\mathrm{E}$ & $\mathrm{X}$ & & $\mathrm{X}$ & & & \\
\hline & Isiro & $\mathrm{E}$ & $\mathrm{X}$ & & $\mathrm{X}$ & & & \\
\hline & Kalemie & $\mathrm{S}$ & $\mathrm{X}$ & & $\mathrm{X}$ & & & \\
\hline & Kamina & $\mathrm{S}$ & $\mathrm{X}$ & & $\mathrm{X}$ & & & \\
\hline & Kananga & $\mathrm{E}$ & $\mathrm{X}$ & & $\mathrm{X}$ & & & \\
\hline & Kikwit & $\mathrm{E}$ & $\mathrm{X}$ & & $\mathrm{X}$ & & & \\
\hline & Kindu & $\mathrm{E}$ & $\mathrm{X}$ & & $\mathrm{X}$ & & & \\
\hline & Kinshasa & $\mathrm{E}$ & $\mathrm{X}$ & & $\mathrm{X}$ & & & \\
\hline & Kisangani & $\mathrm{E}$ & $\mathrm{X}$ & & $\mathrm{X}$ & & & \\
\hline & Kolwezi & $\mathrm{S}$ & $\mathrm{X}$ & & $\mathrm{X}$ & & & \\
\hline & Lubumbashi & $\mathrm{S}$ & $\mathrm{X}$ & & $\mathrm{X}$ & & & \\
\hline & Matadi & $\mathrm{E}$ & $\mathrm{X}$ & & $\mathrm{X}$ & & & \\
\hline & Mbandaka & $\mathrm{E}$ & $\mathrm{X}$ & & $\mathrm{X}$ & & & \\
\hline & Mbuji Mayi & $\mathrm{E}$ & $\mathrm{X}$ & & $\mathrm{X}$ & & & \\
\hline & Tshikapa & $\mathrm{E}$ & $\mathrm{X}$ & & $\mathrm{X}$ & & & \\
\hline & Uvira & $\mathrm{E}$ & $\mathrm{X}$ & & $\mathrm{X}$ & & & \\
\hline & Zongo & $\mathrm{E}$ & $\mathrm{X}$ & & $\mathrm{X}$ & & & \\
\hline Djibouti & Djibouti & $\mathrm{N}$ & & & $\mathrm{X}$ & & & \\
\hline \multirow{2}{*}{ Eritrea } & Asmara & $\mathrm{N}$ & & & & $\mathrm{X}$ & & \\
\hline & Massawa & $\mathrm{N}$ & & & & $\mathrm{X}$ & & \\
\hline \multirow{13}{*}{ Ethiopia } & Addis Ababa & $\mathrm{N}$ & $\mathrm{X}$ & & & $\mathrm{X}$ & $\mathrm{X}$ & $\mathrm{X}$ \\
\hline & Awasa & $\mathrm{N}$ & $\mathrm{X}$ & & & $\mathrm{X}$ & $\mathrm{X}$ & $\mathrm{X}$ \\
\hline & Bahir Dar & $\mathrm{N}$ & $\mathrm{X}$ & & & $\mathrm{X}$ & $\mathrm{X}$ & $\mathrm{X}$ \\
\hline & Dessie & $\mathrm{N}$ & $\mathrm{X}$ & & & $\mathrm{X}$ & $\mathrm{X}$ & $\mathrm{X}$ \\
\hline & Dire Dawa & $\mathrm{N}$ & $\mathrm{X}$ & & & $\mathrm{X}$ & $\mathrm{X}$ & $\mathrm{X}$ \\
\hline & Gambela & $\mathrm{N}$ & $\mathrm{X}$ & & & $\mathrm{X}$ & $\mathrm{X}$ & $\mathrm{X}$ \\
\hline & Gode & $\mathrm{E}$ & $\mathrm{X}$ & & & $\mathrm{X}$ & & $\mathrm{X}$ \\
\hline & Gondar & $\mathrm{N}$ & $\mathrm{X}$ & & & $\mathrm{X}$ & & $\mathrm{X}$ \\
\hline & Jijiga & $\mathrm{E}$ & $\mathrm{X}$ & & & $\mathrm{X}$ & & $\mathrm{X}$ \\
\hline & Jimma & $\mathrm{N}$ & $\mathrm{X}$ & & & $\mathrm{X}$ & $\mathrm{X}$ & $\mathrm{X}$ \\
\hline & Mekele & $\mathrm{N}$ & $\mathrm{X}$ & & & $\mathrm{X}$ & $\mathrm{X}$ & $\mathrm{X}$ \\
\hline & Nekemte & $\mathrm{N}$ & $\mathrm{X}$ & & & $\mathrm{X}$ & $\mathrm{X}$ & \\
\hline & Yabelo & $\mathrm{E}$ & $\mathrm{X}$ & & & & & $\mathrm{X}$ \\
\hline Gabon & Libreville & $\mathrm{N}$ & & & $\mathrm{X}$ & & & \\
\hline Gambia & Banjul & $\mathrm{N}$ & $\mathrm{X}$ & $\mathrm{X}$ & $\mathrm{X}$ & $\mathrm{X}$ & & \\
\hline \multirow[t]{7}{*}{ Ghana } & Accra & $\mathrm{N}$ & $\mathrm{X}$ & $\mathrm{X}$ & $\mathrm{X}$ & $\mathrm{X}$ & & \\
\hline & Bolgatanga & $\mathrm{N}$ & $\mathrm{X}$ & $\mathrm{X}$ & $\mathrm{X}$ & $\mathrm{X}$ & & \\
\hline & Но & $\mathrm{N}$ & $\mathrm{X}$ & & $\mathrm{X}$ & & & \\
\hline & Kumasi & $\mathrm{N}$ & $\mathrm{X}$ & $\mathrm{X}$ & $\mathrm{X}$ & $\mathrm{X}$ & & \\
\hline & Sekondi Takoradi & $\mathrm{N}$ & $\mathrm{X}$ & & $\mathrm{X}$ & & & \\
\hline & Tamale & $\mathrm{N}$ & $\mathrm{X}$ & $\mathrm{X}$ & $\mathrm{X}$ & $\mathrm{X}$ & & \\
\hline & Wa & $\mathrm{N}$ & $\mathrm{X}$ & $\mathrm{X}$ & X & X & & \\
\hline
\end{tabular}


Table A3: Seasonal Regime and Grain Types by Market (3/6)

\begin{tabular}{|c|c|c|c|c|c|c|c|c|}
\hline Country & Market & Season & Maize & Millet & Rice & Sorghum & Teff & Wheat \\
\hline \multirow[t]{4}{*}{ Guinea } & Conakry & $\mathrm{N}$ & & & $\mathrm{X}$ & & & \\
\hline & Kankan & $\mathrm{N}$ & & & $\mathrm{X}$ & & & \\
\hline & Labe & $\mathrm{N}$ & & & $\mathrm{X}$ & & & \\
\hline & Nzerekore & $\mathrm{N}$ & & & $\mathrm{X}$ & & & \\
\hline Guinea Bissau & Bissau & $\mathrm{N}$ & $\mathrm{X}$ & $\mathrm{X}$ & $\mathrm{X}$ & $\mathrm{X}$ & & \\
\hline \multirow[t]{10}{*}{ Kenya } & Eldoret & $\mathrm{E}$ & $\mathrm{X}$ & & & & & \\
\hline & Garissa & $\mathrm{E}$ & $\mathrm{X}$ & & & & & \\
\hline & Kisumu & $\mathrm{E}$ & $\mathrm{X}$ & & & & & \\
\hline & Lodwar & $\mathrm{E}$ & $\mathrm{X}$ & & & & & \\
\hline & Mandera & $\mathrm{E}$ & $\mathrm{X}$ & & & & & \\
\hline & Mombasa & $\mathrm{E}$ & $\mathrm{X}$ & & & & & \\
\hline & Moyale & $\mathrm{E}$ & $\mathrm{X}$ & & & & & \\
\hline & Nairobi & $\mathrm{E}$ & $\mathrm{X}$ & & & & & \\
\hline & Nakuru & $\mathrm{E}$ & $\mathrm{X}$ & & & & & \\
\hline & Wajir & $\mathrm{E}$ & $\mathrm{X}$ & & & & & \\
\hline Lesotho & Maseru & $\mathrm{S}$ & $\mathrm{X}$ & & & & & \\
\hline \multirow{2}{*}{ Liberia } & Gbarnga & $\mathrm{N}$ & & & $\mathrm{X}$ & & & \\
\hline & Monrovia & $\mathrm{N}$ & & & $\mathrm{X}$ & & & \\
\hline \multirow{5}{*}{ Malawi } & Blantyre & S & $\mathrm{X}$ & & & & & \\
\hline & Karonga & $\mathrm{S}$ & $\mathrm{X}$ & & & & & \\
\hline & Lilongwe & $\mathrm{S}$ & $\mathrm{X}$ & & & & & \\
\hline & Mangochi & S & $\mathrm{X}$ & & & & & \\
\hline & Mzuzu & $\mathrm{S}$ & $\mathrm{X}$ & & & & & \\
\hline \multirow[t]{6}{*}{ Mali } & Bamako & $\mathrm{N}$ & & $\mathrm{X}$ & $\mathrm{X}$ & $\mathrm{X}$ & & \\
\hline & Gao & $\mathrm{N}$ & & $\mathrm{X}$ & $\mathrm{X}$ & $\mathrm{X}$ & & \\
\hline & Kayes & $\mathrm{N}$ & & $\mathrm{X}$ & $\mathrm{X}$ & $\mathrm{X}$ & & \\
\hline & Mopti & $\mathrm{N}$ & & $\mathrm{X}$ & $\mathrm{X}$ & $\mathrm{X}$ & & \\
\hline & Segou & $\mathrm{N}$ & & $\mathrm{X}$ & $\mathrm{X}$ & $\mathrm{X}$ & & \\
\hline & Sikasso & $\mathrm{N}$ & & $\mathrm{X}$ & $\mathrm{X}$ & $\mathrm{X}$ & & \\
\hline \multirow[t]{3}{*}{ Mauritania } & Adel Bagrou & $\mathrm{N}$ & & & $\mathrm{X}$ & $\mathrm{X}$ & & \\
\hline & Nouakchott & $\mathrm{N}$ & & & $\mathrm{X}$ & $\mathrm{X}$ & & \\
\hline & Tintane & $\mathrm{N}$ & & & $\mathrm{X}$ & $\mathrm{X}$ & & \\
\hline \multirow[t]{13}{*}{ Mozambique } & Beira & S & $\mathrm{X}$ & & $\mathrm{X}$ & & & \\
\hline & Chimoio & S & $\mathrm{X}$ & & $\mathrm{X}$ & & & \\
\hline & Cuamba & S & $\mathrm{X}$ & & $\mathrm{X}$ & & & \\
\hline & Lichinga & S & $\mathrm{X}$ & & $\mathrm{X}$ & & & \\
\hline & Maputo & S & $\mathrm{X}$ & & $\mathrm{X}$ & & & \\
\hline & Maxixe & S & $\mathrm{X}$ & & $\mathrm{X}$ & & & \\
\hline & Milange & S & $\mathrm{X}$ & & $\mathrm{X}$ & & & \\
\hline & Nacala & S & $\mathrm{X}$ & & $\mathrm{X}$ & & & \\
\hline & Nampula & S & $\mathrm{X}$ & & $\mathrm{X}$ & & & \\
\hline & Pemba & S & $\mathrm{X}$ & & $\mathrm{X}$ & & & \\
\hline & Quelimane & S & $\mathrm{X}$ & & $\mathrm{X}$ & & & \\
\hline & Tete & S & $\mathrm{X}$ & & $\mathrm{X}$ & & & \\
\hline & Xai Xai & S & $\mathrm{X}$ & & $\mathrm{X}$ & & & \\
\hline
\end{tabular}


Table A3: Seasonal Regime and Grain Types by Market (4/6)

\begin{tabular}{|c|c|c|c|c|c|c|c|c|}
\hline Country & Market & Season & Maize & Millet & Rice & Sorghum & Teff & Wheat \\
\hline \multirow[t]{4}{*}{ Namibia } & Katima Mulilo & $\mathrm{S}$ & $\mathrm{X}$ & & & & & \\
\hline & Oshakati & $\mathrm{S}$ & $\mathrm{X}$ & & & & & \\
\hline & Swakopmund & $\mathrm{S}$ & $\mathrm{X}$ & & & & & \\
\hline & Windhoek & $\mathrm{S}$ & $\mathrm{X}$ & & & & & \\
\hline \multirow[t]{7}{*}{ Niger } & Agadez & $\mathrm{N}$ & & $\mathrm{X}$ & & $\mathrm{X}$ & & \\
\hline & Arlit & $\mathrm{N}$ & & $\mathrm{X}$ & & $\mathrm{X}$ & & \\
\hline & Diffa & $\mathrm{N}$ & & $\mathrm{X}$ & & $\mathrm{X}$ & & \\
\hline & Maradi & $\mathrm{N}$ & & $\mathrm{X}$ & & $\mathrm{X}$ & & \\
\hline & Niamey & $\mathrm{N}$ & & $\mathrm{X}$ & & $\mathrm{X}$ & & \\
\hline & Tahoua & $\mathrm{N}$ & & $\mathrm{X}$ & & $\mathrm{X}$ & & \\
\hline & Zinder & $\mathrm{N}$ & & $\mathrm{X}$ & & $\mathrm{X}$ & & \\
\hline \multirow[t]{19}{*}{ Nigeria } & Abuja & $\mathrm{N}$ & $\mathrm{X}$ & $\mathrm{X}$ & $\mathrm{X}$ & $\mathrm{X}$ & & \\
\hline & Akure & $\mathrm{N}$ & $\mathrm{X}$ & $\mathrm{X}$ & $\mathrm{X}$ & $\mathrm{X}$ & & \\
\hline & Benin City & $\mathrm{N}$ & $\mathrm{X}$ & $\mathrm{X}$ & $\mathrm{X}$ & $\mathrm{X}$ & & \\
\hline & Calabar & $\mathrm{N}$ & $\mathrm{X}$ & $\mathrm{X}$ & $\mathrm{X}$ & $\mathrm{X}$ & & \\
\hline & Enugu & $\mathrm{N}$ & $\mathrm{X}$ & $\mathrm{X}$ & $\mathrm{X}$ & $\mathrm{X}$ & & \\
\hline & Gombe & $\mathrm{N}$ & $\mathrm{X}$ & $\mathrm{X}$ & $\mathrm{X}$ & $\mathrm{X}$ & & \\
\hline & Ibadan & $\mathrm{N}$ & $\mathrm{X}$ & $\mathrm{X}$ & $\mathrm{X}$ & $\mathrm{X}$ & & \\
\hline & Ilorin & $\mathrm{N}$ & $\mathrm{X}$ & $\mathrm{X}$ & $\mathrm{X}$ & $\mathrm{X}$ & & \\
\hline & Jos & $\mathrm{N}$ & $\mathrm{X}$ & $\mathrm{X}$ & $\mathrm{X}$ & $\mathrm{X}$ & & \\
\hline & Kaduna & $\mathrm{N}$ & $\mathrm{X}$ & $\mathrm{X}$ & $\mathrm{X}$ & $\mathrm{X}$ & & \\
\hline & Kano & $\mathrm{N}$ & $\mathrm{X}$ & $\mathrm{X}$ & $\mathrm{X}$ & $\mathrm{X}$ & & \\
\hline & Katsina & $\mathrm{N}$ & $\mathrm{X}$ & $\mathrm{X}$ & $\mathrm{X}$ & $\mathrm{X}$ & & \\
\hline & Lagos & $\mathrm{N}$ & $\mathrm{X}$ & $\mathrm{X}$ & $\mathrm{X}$ & $\mathrm{X}$ & & \\
\hline & Lokoja & $\mathrm{N}$ & $\mathrm{X}$ & $\mathrm{X}$ & $\mathrm{X}$ & $\mathrm{X}$ & & \\
\hline & Maiduguri & $\mathrm{N}$ & $\mathrm{X}$ & $\mathrm{X}$ & $\mathrm{X}$ & $\mathrm{X}$ & & \\
\hline & Makurdi & $\mathrm{N}$ & $\mathrm{X}$ & $\mathrm{X}$ & $\mathrm{X}$ & $\mathrm{X}$ & & \\
\hline & Port Harcourt & $\mathrm{N}$ & $\mathrm{X}$ & $\mathrm{X}$ & $\mathrm{X}$ & $\mathrm{X}$ & & \\
\hline & Sokoto & $\mathrm{N}$ & $\mathrm{X}$ & $\mathrm{X}$ & $\mathrm{X}$ & $\mathrm{X}$ & & \\
\hline & Yola & $\mathrm{N}$ & $\mathrm{X}$ & $\mathrm{X}$ & $\mathrm{X}$ & $\mathrm{X}$ & & \\
\hline \multirow[t]{3}{*}{ Rwanda } & Butare & $\mathrm{E}$ & $\mathrm{X}$ & & & $\mathrm{X}$ & & \\
\hline & Gisenyi & $\mathrm{E}$ & $\mathrm{X}$ & & & $\mathrm{X}$ & & \\
\hline & Kigali & $\mathrm{E}$ & $\mathrm{X}$ & & & $\mathrm{X}$ & & \\
\hline \multirow[t]{6}{*}{ Senegal } & Dakar & $\mathrm{N}$ & $\mathrm{X}$ & $\mathrm{X}$ & $\mathrm{X}$ & $\mathrm{X}$ & & \\
\hline & Kaolack & $\mathrm{N}$ & $\mathrm{X}$ & $\mathrm{X}$ & $\mathrm{X}$ & $\mathrm{X}$ & & \\
\hline & Saint Louis & $\mathrm{N}$ & $\mathrm{X}$ & $\mathrm{X}$ & $\mathrm{X}$ & $\mathrm{X}$ & & \\
\hline & Tambacounda & $\mathrm{N}$ & $\mathrm{X}$ & $\mathrm{X}$ & $\mathrm{X}$ & $\mathrm{X}$ & & \\
\hline & Touba & $\mathrm{N}$ & $\mathrm{X}$ & $\mathrm{X}$ & $\mathrm{X}$ & $\mathrm{X}$ & & \\
\hline & Ziguinchor & $\mathrm{N}$ & $\mathrm{X}$ & $\mathrm{X}$ & $\mathrm{X}$ & $\mathrm{X}$ & & \\
\hline \multirow[t]{3}{*}{ Sierra Leone } & Bo & $\mathrm{N}$ & & & $\mathrm{X}$ & & & \\
\hline & Freetown & $\mathrm{N}$ & & & $\mathrm{X}$ & & & \\
\hline & Kabala & $\mathrm{N}$ & & & $\mathrm{X}$ & & & \\
\hline
\end{tabular}


Table A3: Seasonal Regime and Grain Types by Market (5/6)

\begin{tabular}{|c|c|c|c|c|c|c|c|c|}
\hline Country & Market & Season & Maize & Millet & Rice & Sorghum & Teff & Wheat \\
\hline \multirow[t]{8}{*}{ Somalia } & Baidoa & $\mathrm{E}$ & $\mathrm{X}$ & & & $\mathrm{X}$ & & \\
\hline & Beledweyne & $\mathrm{E}$ & $\mathrm{X}$ & & & $\mathrm{X}$ & & \\
\hline & Bosaso & $\mathrm{E}$ & $\mathrm{X}$ & & & $\mathrm{X}$ & & \\
\hline & Galkayo & $\mathrm{E}$ & $\mathrm{X}$ & & & $\mathrm{X}$ & & \\
\hline & Garoowe & $\mathrm{E}$ & $\mathrm{X}$ & & & $\mathrm{X}$ & & \\
\hline & Hargeisa & $\mathrm{E}$ & $\mathrm{X}$ & & & $\mathrm{X}$ & & \\
\hline & Kismayo & $\mathrm{E}$ & $\mathrm{X}$ & & & $\mathrm{X}$ & & \\
\hline & Mogadishu & $\mathrm{E}$ & $\mathrm{X}$ & & & $\mathrm{X}$ & & \\
\hline South Africa & Johannesburg & $\mathrm{S}$ & $\mathrm{X}$ & & & & & \\
\hline \multirow[t]{5}{*}{ South Sudan } & Bor & $\mathrm{N}$ & $\mathrm{X}$ & & & $\mathrm{X}$ & & \\
\hline & Juba & $\mathrm{E}$ & $\mathrm{X}$ & & & $\mathrm{X}$ & & \\
\hline & Malakal & $\mathrm{N}$ & $\mathrm{X}$ & & & $\mathrm{X}$ & & \\
\hline & Rumbek & $\mathrm{N}$ & $\mathrm{X}$ & & & $\mathrm{X}$ & & \\
\hline & Wau & $\mathrm{N}$ & $\mathrm{X}$ & & & $\mathrm{X}$ & & \\
\hline \multirow[t]{11}{*}{ Sudan } & Ad Damazin & $\mathrm{N}$ & & $\mathrm{X}$ & & $\mathrm{X}$ & & $\mathrm{X}$ \\
\hline & Al Fashir & $\mathrm{N}$ & & $\mathrm{X}$ & & $\mathrm{X}$ & & $\mathrm{X}$ \\
\hline & Al Qadarif & $\mathrm{N}$ & & $\mathrm{X}$ & & $\mathrm{X}$ & & $\mathrm{X}$ \\
\hline & El Geneina & $\mathrm{N}$ & & $\mathrm{X}$ & & $\mathrm{X}$ & & $\mathrm{X}$ \\
\hline & El Obeid & $\mathrm{N}$ & & $\mathrm{X}$ & & $\mathrm{X}$ & & $\mathrm{X}$ \\
\hline & Kadugli & $\mathrm{N}$ & & $\mathrm{X}$ & & $\mathrm{X}$ & & $\mathrm{X}$ \\
\hline & Kassala & $\mathrm{N}$ & & $\mathrm{X}$ & & $\mathrm{X}$ & & $\mathrm{X}$ \\
\hline & Khartoum & $\mathrm{N}$ & & $\mathrm{X}$ & & $\mathrm{X}$ & & $\mathrm{X}$ \\
\hline & Kosti & $\mathrm{N}$ & & $\mathrm{X}$ & & $\mathrm{X}$ & & $\mathrm{X}$ \\
\hline & Nyala & $\mathrm{N}$ & & $\mathrm{X}$ & & $\mathrm{X}$ & & $\mathrm{X}$ \\
\hline & Port Sudan & $\mathrm{N}$ & & $\mathrm{X}$ & & $\mathrm{X}$ & & $\mathrm{X}$ \\
\hline Swaziland & Mbabane & $\mathrm{S}$ & $\mathrm{X}$ & & & & & \\
\hline \multirow[t]{15}{*}{ Tanzania } & Arusha & $\mathrm{E}$ & $\mathrm{X}$ & & $\mathrm{X}$ & & & \\
\hline & Bukoba & $\mathrm{E}$ & $\mathrm{X}$ & & $\mathrm{X}$ & & & \\
\hline & Dar es Salaam & $\mathrm{E}$ & $\mathrm{X}$ & & $\mathrm{X}$ & & & \\
\hline & Dodoma & $\mathrm{E}$ & $\mathrm{X}$ & & $\mathrm{X}$ & & & \\
\hline & Iringa & S & $\mathrm{X}$ & & $\mathrm{X}$ & & & \\
\hline & Kigoma & $\mathrm{E}$ & $\mathrm{X}$ & & $\mathrm{X}$ & & & \\
\hline & Mbeya & S & $\mathrm{X}$ & & $\mathrm{X}$ & & & \\
\hline & Mtwara & S & $\mathrm{X}$ & & $\mathrm{X}$ & & & \\
\hline & Musoma & $\mathrm{E}$ & $\mathrm{X}$ & & $\mathrm{X}$ & & & \\
\hline & Mwanza & $\mathrm{E}$ & $\mathrm{X}$ & & $\mathrm{X}$ & & & \\
\hline & Singida & $\mathrm{E}$ & $\mathrm{X}$ & & $\mathrm{X}$ & & & \\
\hline & Songea & $\mathrm{S}$ & X & & $\mathrm{X}$ & & & \\
\hline & Sumbawanga & $\mathrm{S}$ & $\mathrm{X}$ & & $\mathrm{X}$ & & & \\
\hline & Tabora & $\mathrm{E}$ & $\mathrm{X}$ & & $\mathrm{X}$ & & & \\
\hline & Tanga & $\mathrm{E}$ & $\mathrm{X}$ & & $\mathrm{X}$ & & & \\
\hline \multirow[t]{2}{*}{ Togo } & Kara & $\mathrm{N}$ & $\mathrm{X}$ & & $\mathrm{X}$ & $\mathrm{X}$ & & \\
\hline & Lome & $\mathrm{N}$ & $\mathrm{X}$ & & $\mathrm{X}$ & $\mathrm{X}$ & & \\
\hline
\end{tabular}


Table A3: Seasonal Regime and Grain Types by Market (6/6)

\begin{tabular}{|c|c|c|c|c|c|c|c|c|}
\hline Country & Market & Season & Maize & Millet & Rice & Sorghum & Teff & Wheat \\
\hline \multirow[t]{7}{*}{ Uganda } & Arua & $\mathrm{E}$ & $\mathrm{X}$ & & & & & \\
\hline & Gulu & $\mathrm{E}$ & $\mathrm{X}$ & & & $\mathrm{X}$ & & \\
\hline & Jinja & $\mathrm{E}$ & $\mathrm{X}$ & & & $\mathrm{X}$ & & \\
\hline & Kampala & $\mathrm{E}$ & $\mathrm{X}$ & & & $\mathrm{X}$ & & \\
\hline & Lira & $\mathrm{E}$ & $\mathrm{X}$ & & & $\mathrm{X}$ & & \\
\hline & Masindi & $\mathrm{E}$ & $\mathrm{X}$ & & & $\mathrm{X}$ & & \\
\hline & Mbarara & $\mathrm{E}$ & $\mathrm{X}$ & & & $\mathrm{X}$ & & \\
\hline \multirow[t]{8}{*}{ Zambia } & Chipata & S & $\mathrm{X}$ & & & & & \\
\hline & Kabwe & S & $\mathrm{X}$ & & & & & \\
\hline & Kasama & S & $\mathrm{X}$ & & & & & \\
\hline & Kitwe & S & $\mathrm{X}$ & & & & & \\
\hline & Livingstone & S & $\mathrm{X}$ & & & & & \\
\hline & Lusaka & $\mathrm{S}$ & $\mathrm{X}$ & & & & & \\
\hline & Mongu & $\mathrm{S}$ & $\mathrm{X}$ & & & & & \\
\hline & Solwezi & S & $\mathrm{X}$ & & & & & \\
\hline \multirow[t]{6}{*}{ Zimbabwe } & Bulawayo & $\mathrm{S}$ & $\mathrm{X}$ & & & & & \\
\hline & Harare & S & $\mathrm{X}$ & & & & & \\
\hline & Hwange & S & $\mathrm{X}$ & & & & & \\
\hline & Masvingo & S & $\mathrm{X}$ & & & & & \\
\hline & Mutare & $\mathrm{S}$ & $\mathrm{X}$ & & & & & \\
\hline & Total & & 180 & 64 & 126 & 110 & 9 & 23 \\
\hline
\end{tabular}


Table A4: Primary and Secondary Data Sources by Country

\begin{tabular}{|c|c|c|c|c|}
\hline Country & Markets & Series & Primary Source & Secondary Source \\
\hline Angola & 1 & 1 & National Institute of Statistics & \\
\hline Benin & 4 & 9 & Min. of Ag., Livestock, and Fisheries & FAO GIEWS \\
\hline Botswana & 1 & 1 & Central Statistics Office & BIDPA \\
\hline Burkina Faso & 4 & 8 & Afrique Verte & FAO GIEWS \\
\hline Burundi & 3 & 6 & World Food Programme & USAID FEWS NET \\
\hline Cameroon & 4 & 8 & National Institute of Statistics & FAO GIEWS \\
\hline C.A.R. & 3 & 6 & World Food Programme & WFP VAM \\
\hline Chad & 4 & 10 & USAID FEWS NET & FAO GIEWS \\
\hline Congo & 3 & 3 & World Food Programme & WFP VAM \\
\hline Côte d'Ivoire & 6 & 12 & World Food Programme & WFP VAM \\
\hline D.R. Congo & 22 & 44 & FAO-DRC and Min. of Ag. and Rural Dev't & \\
\hline Djibouti & 1 & 1 & Dept. of Statistics and Demographic Studies & USAID FEWS NET \\
\hline Eritrea & 2 & 2 & UN OCHA Eritrea & FAO GIEWS \\
\hline Ethiopia & 13 & 46 & $\begin{array}{l}\text { Ethiopian Grain Trade Enterprise; } \\
\text { World Food Programme }\end{array}$ & $\begin{array}{l}\text { FAO GIEWS; } \\
\text { WFP VAM }\end{array}$ \\
\hline Gabon & 1 & 1 & Ministry of Economy and Planning & FAO GIEWS \\
\hline Gambia & 1 & 4 & Bureau of Statistics & WFP VAM \\
\hline Ghana & 7 & 24 & Ministry of Food and Agriculture & FAO GIEWS; WFP VAM \\
\hline Guinea & 4 & 4 & World Food Programme & WFP VAM \\
\hline Guinea Bissau & 1 & 4 & World Food Programme & WFP VAM \\
\hline Kenya & 10 & 10 & $\begin{array}{l}\text { Min. of Ag., Livestock, \& Fish.; Nat'l } \\
\text { Drought Managem. Authority; RATIN }\end{array}$ & $\begin{array}{l}\text { USAID FEWS NET; } \\
\text { FAO GIEWS }\end{array}$ \\
\hline Lesotho & 1 & 1 & Bureau of Statistics & FAO GIEWS \\
\hline Liberia & 2 & 2 & World Food Programme & WFP VAM \\
\hline Malawi & 5 & 5 & Ministry of Agriculture and Food Security & \\
\hline Mali & 6 & 18 & Afrique Verte & FAO GIEWS \\
\hline Mauritania & 3 & 6 & World Food Programme & WFP VAM \\
\hline Mozambique & 13 & 26 & Ministry of Agriculture & WFP VAM \\
\hline Namibia & 4 & 4 & Namibia Statistics Agency & \\
\hline Niger & 7 & 14 & Min. of Trade and Private Sector Promotion & FAO GIEWS; WFP VAM \\
\hline Nigeria & 19 & 76 & National Bureau of Statistics & D. Donaldson \\
\hline Rwanda & 3 & 6 & Min. of Ag. and Animal Resources & WFP VAM \\
\hline Senegal & 6 & 24 & Food Security Commission & WFP VAM \\
\hline Sierra Leone & 3 & 3 & World Food Programme & WFP VAM \\
\hline Somalia & 8 & 16 & Food Security and Nutrition Analysis Unit & \\
\hline South Africa & 1 & 1 & South African Futures Exchange & FAO GIEWS \\
\hline South Sudan & 5 & 10 & World Food Programme & WFP VAM \\
\hline Sudan & 11 & 33 & $\begin{array}{l}\text { World Food Programme; } \\
\text { Food Security Information for Action }\end{array}$ & $\begin{array}{l}\text { WFP VAM } \\
\text { FAO GIEWS }\end{array}$ \\
\hline Swaziland & 1 & 1 & Ministry of Agriculture & WFP VAM \\
\hline Tanzania & 15 & 30 & Min. of Industry, Trade, and Marketing & WFP VAM \\
\hline Togo & 2 & 6 & Min. of Ag., Livestock, and Fisheries & FAO GIEWS \\
\hline Uganda & 7 & 13 & $\begin{array}{l}\text { Infotrade Uganda; } \\
\text { Farmgain Africa }\end{array}$ & $\begin{array}{l}\text { WFP VAM; } \\
\text { USAID FEWS NET }\end{array}$ \\
\hline Zambia & 8 & 8 & Central Statistics Office & WFP VAM \\
\hline Zimbabwe & 5 & 5 & World Food Programme & WFP VAM \\
\hline Total & 230 & 512 & & \\
\hline
\end{tabular}




\section{A3 Data: Retail and Wholesale Price Series}

390 (76 percent) of the 512 price series are identified as retail price series for quantities ranging from 0.5 to $3.5 \mathrm{~kg}$, while the remainder are identified as wholesale price series for quantities ranging from 50 to $100 \mathrm{~kg}$. Table A5 on the next page reports results from a statistical test of 37 series from 17 markets in 5 countries for which both "retail" and "wholesale" prices are available. My null hypothesis is that retail prices and wholesale prices are not significantly different, which is consistent with interviews of market participants suggesting that separate retail and wholesale markets typically do not exist and that prices per kilogram often do not vary with quantity sold. To test this hypothesis, I subtract each wholesale price series from its respective retail price series and then regress each resulting series of differences on a constant. I fail to reject the null for 9 of 37 series (24.3 percent), I find retail prices significantly greater than wholesale prices for 23 of 37 series (62.2 percent), and I find wholesale prices significantly greater than retail prices for 5 of 37 series (13.5 percent). Interestingly, all 9 of the 9 series from 4 large commercial capital cities have retail price series significantly greater than wholesale price series, suggesting that more sophisticated, separate markets may exist in these environments. These 4 cities all have populations over 1 million, whereas the remaining 28 series come from cities with populations less than 500,000. Without the 9 series from the large cities, exactly 50 percent of the remaining 28 series have retail prices significantly greater than wholesale prices while 50 percent have retail prices not different or significantly smaller than wholesale price series.

While I cannot reject my null hypothesis of equality across all markets, there do appear to be some individual markets with a significant difference between retail and wholesale prices. This would be problematic for my estimation of trade costs in cases where a market with a wholesale price series is directly connected to a market with a retail price series. Fortunately, such cases are few - only 60 of the 413 links in my network (14.5 percent). Of these, only 29 (7.0 percent) involve a city with a population larger than 500,000. In table A6, I compare my estimated trade costs along these 29 links to estimated trade costs along similar nearby links with identical series types (wholesale-wholesale or retail-retail). Although direct comparisons are difficult to make due to the particularities of each link, the costs per tonne-km of the 29 potentially affected links do not appear to be systematically larger than those of their comparison links. The estimated trade costs along the 29 potentially affected links are also all much higher than the counterfactual trade cost of $\$ 0.05 / \mathrm{t}-\mathrm{km}$, suggesting that any small bias in my trade cost estimates for these links due to retail-wholesale price discrepancies would not affect my results significantly. 
Table A5: Statistical Test of Retail-Wholesale Price Difference

\begin{tabular}{|c|c|c|c|c|c|c|c|}
\hline Market & Country & Crop & Observ. & Coefficient & Std. Error & Result & Large City \\
\hline \multirow[t]{2}{*}{ Ad Damazin } & \multirow[t]{2}{*}{ Sudan } & Millet & 79 & 0.0935 & $(0.0143)$ & + & \\
\hline & & Sorghum & 86 & 0.0629 & $(0.0067)$ & + & \\
\hline \multirow[t]{4}{*}{ Addis Ababa } & \multirow[t]{4}{*}{ Ethiopia } & Maize & 52 & 0.2525 & $(0.0173)$ & + & $\mathrm{X}$ \\
\hline & & Sorghum & 64 & 0.1875 & $(0.0132)$ & + & $\mathrm{X}$ \\
\hline & & Teff & 72 & 0.0373 & $(0.0036)$ & + & $\mathrm{X}$ \\
\hline & & Wheat & 72 & 0.0812 & $(0.0066)$ & + & $\mathrm{X}$ \\
\hline \multirow[t]{2}{*}{ Agadez } & \multirow[t]{2}{*}{ Niger } & Millet & 76 & $-1.49 \mathrm{E}-04$ & $(0.0031)$ & $=$ & \\
\hline & & Sorghum & 49 & $-4.79 \mathrm{E}-04$ & $(0.0049)$ & $=$ & \\
\hline \multirow[t]{2}{*}{ Al Fashir } & \multirow[t]{2}{*}{ Sudan } & Millet & 82 & 0.0458 & $(0.0110)$ & + & \\
\hline & & Sorghum & 60 & 0.1203 & $(0.0147)$ & + & \\
\hline \multirow[t]{2}{*}{ Bahir Dar } & \multirow[t]{2}{*}{ Ethiopia } & Teff & 71 & -0.0106 & $(0.0048)$ & - & \\
\hline & & Wheat & 67 & 0.0263 & $(0.0063)$ & + & \\
\hline \multirow[t]{2}{*}{ Dar es Salaam } & \multirow[t]{2}{*}{ Tanzania } & Maize & 100 & 0.1646 & $(0.0090)$ & + & $\mathrm{X}$ \\
\hline & & Rice & 79 & 0.0937 & $(0.0118)$ & + & $\mathrm{X}$ \\
\hline \multirow[t]{3}{*}{ Dire Dawa } & \multirow[t]{3}{*}{ Ethiopia } & Maize & 41 & 0.1972 & $(0.0162)$ & + & \\
\hline & & Sorghum & 55 & 0.0360 & $(0.0081)$ & + & \\
\hline & & Teff & 65 & 0.0775 & $(0.0175)$ & + & \\
\hline \multirow[t]{2}{*}{ El Geneina } & \multirow[t]{2}{*}{ Sudan } & Millet & 72 & -0.0153 & $(0.0086)$ & $=$ & \\
\hline & & Sorghum & 66 & -0.0259 & $(0.0107)$ & - & \\
\hline \multirow[t]{2}{*}{ El Obeid } & \multirow[t]{2}{*}{ Sudan } & Millet & 109 & 0.0171 & $(0.0086)$ & $=$ & \\
\hline & & Sorghum & 94 & 0.0228 & $(0.0065)$ & + & \\
\hline \multirow[t]{3}{*}{ Kadugli } & \multirow[t]{3}{*}{ Sudan } & Millet & 71 & 0.0345 & $(0.0185)$ & $=$ & \\
\hline & & Sorghum & 103 & 0.0176 & $(0.0059)$ & + & \\
\hline & & Wheat & 43 & -0.0134 & $(0.0204)$ & $=$ & \\
\hline Kampala & Uganda & Maize & 118 & 0.0840 & $(0.0025)$ & + & $\mathrm{X}$ \\
\hline \multirow{2}{*}{ Maradi } & \multirow{2}{*}{ Niger } & Millet & 76 & $-1.17 \mathrm{E}-04$ & $(0.0024)$ & $=$ & \\
\hline & & Sorghum & 76 & -0.0098 & $(0.0033)$ & - & \\
\hline Mekele & Ethiopia & Teff & 66 & 0.0362 & $(0.0097)$ & + & \\
\hline \multirow[t]{2}{*}{ Niamey } & \multirow[t]{2}{*}{ Niger } & Millet & 76 & 0.0593 & $(0.0030)$ & + & $\mathrm{X}$ \\
\hline & & Sorghum & 76 & 0.0865 & $(0.0032)$ & + & $\mathrm{X}$ \\
\hline \multirow[t]{2}{*}{ Nyala } & \multirow[t]{2}{*}{ Sudan } & Millet & 72 & 0.0136 & $(0.0101)$ & $=$ & \\
\hline & & Sorghum & 70 & 0.0139 & $(0.0058)$ & + & \\
\hline Port Sudan & Sudan & Millet & 91 & 0.0788 & $(0.0183)$ & + & \\
\hline & & Sorghum & 96 & 0.0086 & $(0.0037)$ & + & \\
\hline & & Wheat & 55 & 0.0064 & $(0.0174)$ & $=$ & \\
\hline Zinder & Niger & Millet & 76 & -0.0328 & $(0.0037)$ & - & \\
\hline & & Sorghum & 52 & -0.0213 & $(0.0039)$ & - & \\
\hline
\end{tabular}

Note: Result column indicates whether the retail price is greater than $(+)$, less than $(-)$, or not different from $(=)$ the wholesale price at 5 percent significance. 
Table A6: Estimated Trade Costs Along Potentially Affected Links and Nearby Comparison Links

\begin{tabular}{|c|c|c|c|c|c|}
\hline Potentially Affected Link & $\tau_{m n}$ & $/ \mathrm{t}-\mathrm{km}$ & Comparison Link & $\tau_{m n}$ & $/ \mathrm{t}-\mathrm{km}$ \\
\hline Monrovia LR - Bo SL & 0.238 & 0.667 & Freetown SL - Conakry GN & 0.398 & 1.279 \\
\hline Bamako ML - Odienne CI & 0.091 & 0.225 & Odienne CI - Kankan GN & 0.120 & 0.398 \\
\hline Bamako ML - Kankan GN & 0.160 & 0.429 & Labe GN - Tambac. SN & 0.179 & 0.424 \\
\hline Bamako ML - Adel Bagrou MR & 0.110 & 0.256 & Kayes ML - Tambac. SN & 0.088 & 0.309 \\
\hline Bamako ML - Tintane MR & 0.394 & 0.560 & & & \\
\hline Ouaga. BF - Kara TG & 0.132 & 0.248 & Ouaga. BF - Bolgatanga GH & 0.096 & 0.451 \\
\hline Niamey NE - Gao ML & 0.127 & 0.284 & Niamey NE - Malanville BJ & 0.083 & 0.281 \\
\hline Niamey NE - Fada Ng. BF & 0.152 & 0.520 & & & \\
\hline Accra GH - Lome TG & 0.249 & 1.298 & Cot & 0.188 & 1.563 \\
\hline Kumasi GH - Abeng. CI & 0.161 & 0.606 & Abeng. CI - Bouake $\mathrm{CI}^{d}$ & 0.211 & 0.611 \\
\hline Abidjan CI - Sek.-Tak. GH & 0.163 & 0.509 & Abidjan CI - Bouake $\mathrm{CI}^{d}$ & 0.147 & 0.418 \\
\hline Bouake CI - Sikasso ML & 0.061 & 0.123 & Sikasso ML - Bobo Diou. BF & 0.022 & 0.122 \\
\hline Luanda AO - Matadi CD & 1.074 & 1.168 & $\mathrm{CD}$ - Kinshasa $\mathrm{CD}^{d}$ & 0.366 & 1.012 \\
\hline Luanda AO - Kinshasa CD & 1.041 & 1.960 & & & \\
\hline Jo'burg ZA - Mase & 0.071 & 0.168 & $\mathrm{ZA}-\mathrm{Mb}$ & 0.089 & 0.251 \\
\hline Jo'burg ZA - Gaborone BW & 0.114 & 0.312 & Bulaw. ZW - Masv. ZW ${ }^{d}$ & 0.118 & 0.415 \\
\hline Jo'burg ZA - Maputo MZ & 0.213 & 0.391 & & & \\
\hline Jo'burg ZA - Windhoek NA & 0.260 & 0.190 & & & \\
\hline Jo'burg ZA - Bulaw. ZW & 0.081 & 0.093 & & & \\
\hline Jo'burg ZA - Masv. ZW & 0.111 & 0.134 & & & \\
\hline Maputo MZ - Mbabane SZ & 0.100 & 0.451 & Maputo MZ - Xai Xai MZ ${ }^{d}$ & 0.105 & 0.485 \\
\hline Nairobi KE - Garissa KE ${ }^{d}$ & 0.202 & 0.553 & Garissa KE - Wajir $\mathrm{KE}^{d}$ & 0.155 & 0.480 \\
\hline Mombasa KE - Garissa KE ${ }^{d}$ & 0.151 & 0.327 & & & \\
\hline Kigali RW - Mwanza TZ & 0.079 & 0.147 & Kigali RW - Mbarara UG & 0.141 & 0.582 \\
\hline Kampala UG - Bukoba TZ & 0.136 & 0.456 & Kigali RW - Mbarara UG & 0.141 & 0.582 \\
\hline Khartoum SD - Ad Dam. SD ${ }^{d}$ & 0.112 & 0.211 & Ad Dam. SD - Kosti SD ${ }^{d}$ & 0.077 & 0.220 \\
\hline Khartoum SD - Kosti $\mathrm{SD}^{d}$ & 0.062 & 0.196 & Khartoum SD - Al Qad. SD & 0.066 & 0.186 \\
\hline Asmara ER - Mekele ET & 0.560 & 1.801 & Asmara ER - Gondar ET & 0.611 & 1.136 \\
\hline Asmara ER - Kassala SD & 0.689 & 1.610 & & & \\
\hline \multicolumn{6}{|l|}{ Note: ${ }^{d}$ Domestic link } \\
\hline
\end{tabular}




\section{A4 Model: Derivation of Inverse Demand and Welfare}

In this section I derive expressions for inverse demand and welfare (indirect utility). The consumer's utility maximization problem is as follows:

$$
\max _{\left\{Q_{i m t}\right\}_{i \in I_{m}}, X_{m t}} \frac{\theta_{m t}\left(\left[\sum_{i \in I_{m}} \alpha_{i m}^{1 / \sigma} Q_{i m t}^{(\sigma-1) / \sigma}\right]^{\sigma /(\sigma-1)}\right)^{1+\frac{1}{\epsilon}}}{1+\frac{1}{\epsilon}}+X_{m t} \text { such that } \sum_{i \in I_{m}} P_{i m t} Q_{i m t}+X_{m t} \leq Y_{m t}
$$

The first order condition for $X_{m t}$ is $\lambda=1$ where $\lambda$ is the Lagrange multiplier. The first order condition for any grain $i$ is:

$$
\theta_{m t}\left(\left[\sum_{j \in I_{m}} \alpha_{j m}^{1 / \sigma} Q_{j m t}^{(\sigma-1) / \sigma}\right]^{\sigma /(\sigma-1)}\right)^{1 / \epsilon}\left[\sum_{j \in I_{m}} \alpha_{j m}^{1 / \sigma} Q_{j m t}^{(\sigma-1) / \sigma}\right]^{1 /(\sigma-1)} \quad \alpha_{i m}^{1 / \sigma} Q_{i m t}^{-1 / \sigma}=\lambda P_{i m t}=P_{i m t}
$$

Rearranging and letting $\theta_{m t}^{-\epsilon}=A_{m} N_{m t}$ gives the inverse demand function for any grain $i$ :

$$
P_{i m t}=\frac{\alpha_{i m}^{1 / \sigma}}{Q_{i m t}^{1 / \sigma}} * \frac{Q_{m t}^{1 / \sigma+1 / \epsilon}}{\left(A_{m} N_{m t}\right)^{1 / \epsilon}}
$$

where $Q_{m t}=\left[\sum_{i \in I_{m}} \alpha_{i m}^{1 / \sigma} Q_{i m t}^{(\sigma-1) / \sigma}\right]^{\sigma /(\sigma-1)}$ is the CES quantity index (the grain composite).

I next turn to deriving an expression for welfare (indirect utility). Rearranging the previous expression gives the demand function for any grain $i$ :

$$
Q_{i m t}=\frac{\alpha_{i m}}{P_{i m t}^{\sigma}} * \frac{Q_{m t}^{1+\sigma / \epsilon}}{\left(A_{m} N_{m t}\right)^{\sigma / \epsilon}}
$$

Taking the ratio of any two such equations for grains $j$ and $i$ gives:

$$
\begin{gathered}
\frac{Q_{j m t}}{Q_{i m t}}=\frac{\alpha_{j m} P_{i m t}^{\sigma}}{\alpha_{i m} P_{j m t}^{\sigma}} \\
\Rightarrow Q_{j m t}=\frac{\alpha_{j m} Q_{i m t} P_{i m t}^{\sigma}}{\alpha_{i m} P_{j m t}^{\sigma}} \\
\Rightarrow P_{j m t} Q_{j m t}=\frac{\alpha_{j m} P_{j m t}^{1-\sigma} Q_{i m t} P_{i m t}^{\sigma}}{\alpha_{i m}} \\
\Rightarrow \sum_{j \in I_{m}} P_{j m t} Q_{j m t}=\frac{Q_{i m t} P_{i m t}^{\sigma}}{\alpha_{i m}} \sum_{j \in I_{m}} \alpha_{j m} P_{j m t}^{1-\sigma} \\
\Rightarrow Q_{i m t}=\frac{\alpha_{i m} \sum_{j \in I_{m}} P_{j m t} Q_{j m t}}{P_{i m t}^{\sigma} \sum_{j \in I_{m}} \alpha_{j m} P_{j m t}^{1-\sigma}}
\end{gathered}
$$

I can use this last expression to rewrite the grain composite as follows:

$$
\begin{gathered}
Q_{m t}=\left[\sum_{i \in I_{m}} \alpha_{i m}^{1 / \sigma} Q_{i m t}^{(\sigma-1) / \sigma}\right]^{\sigma /(\sigma-1)}=\frac{\sum_{j \in I_{m}} P_{j m t} Q_{j m t}}{\sum_{j \in I_{m}} \alpha_{j m} P_{j m t}^{1-\sigma}}\left[\sum_{i \in I_{m}} \alpha_{i m}^{1 / \sigma}\left(\frac{\alpha_{i m}}{P_{i m t}^{\sigma}}\right)^{(\sigma-1) / \sigma}\right]^{\sigma /(\sigma-1)} \\
\Rightarrow Q_{m t}=\frac{\sum_{j \in I_{m}} P_{j m t} Q_{j m t}}{\sum_{j \in I_{m}} \alpha_{j m} P_{j m t}^{1-\sigma}}\left[\sum_{i \in I_{m}} \alpha_{i m} P_{i m t}^{1-\sigma}\right]^{\sigma /(\sigma-1)}
\end{gathered}
$$




$$
\Rightarrow Q_{m t}=\frac{\sum_{j \in I_{m}} P_{j m t} Q_{j m t}}{\left[\sum_{j \in I_{m}} \alpha_{j m} P_{j m t}^{1-\sigma}\right]^{1 /(1-\sigma)}}=\frac{\sum_{j \in I_{m}} P_{j m t} Q_{j m t}}{P_{m t}}
$$

where $P_{m t}=\left[\sum_{j \in I_{m}} \alpha_{j m} P_{j m t}^{1-\sigma}\right]^{1 /(1-\sigma)}$ is the CES grain price index. Plugging this into the demand function derived above gives:

$$
\begin{gathered}
Q_{i m t}=\frac{\alpha_{i m}}{P_{i m t}^{\sigma}} * \frac{\left(\frac{\sum_{j \in I_{m}} P_{j m t} Q_{j m t}}{P_{m t}}\right)^{1+\sigma / \epsilon}}{\left(A_{m} N_{m t}\right)^{\sigma / \epsilon}} \\
\Rightarrow P_{i m t} Q_{i m t}=\alpha_{i m} P_{i m t}^{1-\sigma} * \frac{\left(\frac{\sum_{j \in I_{m}} P_{j m t} Q_{j m t}}{P_{m t}}\right)^{1+\sigma / \epsilon}}{\left(A_{m} N_{m t}\right)^{\sigma / \epsilon}} \\
\Rightarrow \sum_{j \in I_{m}} P_{j m t} Q_{j m t}=\sum_{j \in I_{m}} \alpha_{j m} P_{j m t}^{1-\sigma} * \frac{\left(\frac{\sum_{j \in I_{m}} P_{j m t} Q_{j m t}}{P_{m t}}\right)^{1+\sigma / \epsilon}}{\left(A_{m} N_{m t}\right)^{\sigma / \epsilon}} \\
\Rightarrow\left(\sum_{j \in I_{m}} P_{j m t} Q_{j m t}\right)^{-\sigma / \epsilon}=\frac{P_{m t}^{-\sigma(1+1 / \epsilon)}}{\left(A_{m} N_{m t}\right)^{\sigma / \epsilon}} \\
\Rightarrow \sum_{j \in I_{m}} P_{j m t} Q_{j m t}=A_{m} N_{m t} P_{m t}^{\epsilon+1}
\end{gathered}
$$

Plugging this into the expression for the quantity index derived above gives:

$$
Q_{m t}=A_{m} N_{m t} P_{m t}^{\epsilon}
$$

I next plug the two previous expressions into the utility function to obtain indirect utility $V_{m t}$ :

$$
\begin{gathered}
U_{m t}=\theta_{m t} \frac{Q_{m t}^{1+\frac{1}{\epsilon}}}{1+\frac{1}{\epsilon}}+X_{m t}=\left(A_{m} N_{m t}\right)^{-\frac{1}{\epsilon}} \frac{Q_{m t}^{1+\frac{1}{\epsilon}}}{1+\frac{1}{\epsilon}}+Y_{m t}-\sum_{j \in I_{m}} P_{j m t} Q_{j m t} \\
\Rightarrow V_{m t}=\frac{\epsilon}{\epsilon+1} A_{m} N_{m t} P_{m t}^{\epsilon+1}+Y_{m t}-A_{m} N_{m t} P_{m t}^{\epsilon+1} \\
\Rightarrow V_{m t}=Y_{m t}-\frac{1}{\epsilon+1} A_{m} N_{m t} P_{m t}^{\epsilon+1}
\end{gathered}
$$

This last expression is identical to equation 5 in the main paper. 


\section{A5 Estimation: Demand Parameters}

To estimate the elasticity of substitution $(\sigma)$ I use standard techniques for CES utility. The expenditure share on grain $i\left(s_{i m t}\right)$ is given by:

$$
s_{i m t} \equiv \frac{P_{i m t} Q_{i m t}}{\sum_{j \in I_{m}} P_{j m t} Q_{j m t}}=\frac{\alpha_{i m} P_{i m t}^{1-\sigma}}{\sum_{j \in I_{m}} \alpha_{j m} P_{j m t}^{1-\sigma}}
$$

Taking the natural logarithm of both sides gives:

$$
\ln s_{i m t}=\ln \alpha_{i m}+(1-\sigma) \ln P_{i m t}-\ln \left(\sum_{j \in I_{m}} \alpha_{j m} P_{j m t}^{1-\sigma}\right)
$$

Using data on consumption and prices I then run the following regression:

$$
\ln s_{i m t}=\mu_{i m}+\beta \ln P_{i m t}+\mu_{m t}+v_{i m t}
$$

where $\mu_{i m}$ and $\mu_{m t}$ are crop-country and country-year fixed effects and $v_{i m t}$ is an error term. My estimate for $\sigma$ is then $1-\beta$.

Results are summarized in table A7. A simple OLS regression yields an estimate of $\sigma=0.51$, with a clustered standard error of 0.21 . However, OLS is unlikely to yield consistent estimates of $\beta$ since equilibrium prices are affected by unobserved demand shocks, i.e. $\operatorname{Cov}\left(P_{i m t}, v_{i m t}\right) \neq 0$. I therefore use an instrumental variables regression with the landed world price as the instrument. I define the landed world price $Z_{i m t}$ as the world price plus the average price difference between the world price and the country's largest city, which is a lower bound on trade costs. Crops with no world price (millet, teff) are excluded. The identifying assumption is that $\operatorname{Cov}\left(Z_{i m t}, v_{i m t}\right)=0$, i.e. that the landed world price only affects the expenditure share through its effect on local prices.

The first stage regression is as follows:

$$
\ln P_{i m t}=\mu_{i m}+\gamma \ln Z_{i m t}+\mu_{m t}+v_{i m t}
$$

which yields an estimate of $\gamma=0.495$ with an unclustered standard error of 0.073 and a standard error of 0.202 when clustering by country-crop (387 observations, 56 clusters). There is thus a strong positive correlation between the landed world price and local prices in Africa, which is to be expected given Africa's position as a net grain importer. The first stage F statistic is 45.7 without clustering and 6.0 with clustering by country-crop.

Table A7: Elasticity Estimates

\begin{tabular}{lcccc}
\hline & OLS: $\sigma$ & OLS: $\epsilon$ & IV: $\sigma$ & IV: $\epsilon$ \\
\hline Estimate & 0.51 & -0.256 & 0.90 & -0.136 \\
& $(0.21)$ & $(0.071)$ & $(0.18)$ & $(0.116)$ \\
1st Stage F Stat & & & 6.0 & 70.3 \\
Observations & 463 & 289 & $387^{b}$ & 289 \\
Clustered Errors & country-crop & country & country-crop & country \\
Clusters & 67 & 28 & 56 & 28 \\
\hline
\end{tabular}

Note: Robust standard errors in () and F statistic clustered as indicated.

${ }^{b}$ Crops with no world price (millet, teff) excluded.

The full instrumental variables regression yields an estimate of $\sigma=0.90$, with a clustered standard error of 0.18 and a 95 percent confidence interval of $[0.56,1.25]$. The estimated $\sigma$ is 
close to the Cobb-Douglas benchmark of $\sigma=1$, implying that expenditure shares are only slightly affected by price changes, if at all.

For a particular $\sigma$ (e.g. $\sigma=1$ in my baseline case, $\sigma=3$ in my robustness checks) I proceed to estimate the associated $\alpha_{i m}$ by estimating individual regressions for each country $m$ of the form:

$$
\left(\ln s_{i m t}-(1-\sigma) \ln P_{i m t}\right)=\mu_{i m}+\mu_{m t}+v_{i m t}
$$

I back out the $\alpha_{i m}$ parameters from the coefficients on the fixed effect indicator variables $\mu_{i m}$. To compute standard errors, I bootstrap by resampling the data with replacement (10,000 iterations).

To estimate the price elasticity of grain demand $(\epsilon)$, I first compute the price and quantity indices for the relevant $\sigma$ and its of $\alpha_{i m}$. For my baseline case, I set $\sigma=1$, so I use the CobbDouglas price and quantity indices: $P_{m t}=\prod_{i \in I_{m}} P_{i m t}^{\alpha_{i m}}$ and $Q_{m t}=\prod_{i \in I_{m}}\left(\frac{Q_{i m t}}{\alpha_{i m}}\right)^{\alpha_{i m}}$. As shown in the previous section, overall grain demand in terms of price and quantity indices is given by:

$$
Q_{m t}=A_{m} N_{m t} P_{m t}^{\epsilon}
$$

Letting $q_{m t}$ denote per-capita grain consumption gives:

$$
\begin{gathered}
q_{m t} \equiv \frac{Q_{m t}}{N_{m t}}=A_{m} P_{m t}^{\epsilon} \\
\Rightarrow \ln q_{m t}=\ln A_{m}+\epsilon \ln P_{m t}
\end{gathered}
$$

I then run the following regression:

$$
\ln q_{m t}=\mu_{m}+\epsilon \ln P_{m t}+v_{m t}
$$

where $\mu_{m}$ are country fixed effects. A simple OLS regression yields an estimate of $\epsilon=-0.256$ with a clustered standard error of 0.071 . However, once again OLS is unlikely to yield consistent estimates of $\epsilon$ since equilibrium prices are affected by unobserved demand shocks, i.e. $\operatorname{Cov}\left(P_{m t}, v_{m t}\right) \neq 0$. I therefore again use an instrumental variables regression with the landed world price index $Z_{m t}$ as the instrument (local prices of millet and teff are used in this index as necessary). The identifying assumption is that $\operatorname{Cov}\left(Z_{m t}, v_{m t}\right)=0$, i.e. that the landed world price index only affects the expenditure share through its effect on local prices.

The first stage regression is as follows:

$$
\ln P_{m t}=\mu_{m}+\gamma \ln Z_{m t}+v_{m t}
$$

which yields an estimate of $\gamma=1.02$ with a standard error of 0.12 when clustering by country (289 observations, 28 clusters). The clustered first stage $\mathrm{F}$ statistic is 70.3 . Once again there is a strong positive correlation between the world price indices and the local price indices in Africa.

The full instrumental variables regression yields an estimate of $\epsilon=-0.136$ with a clustered standard error of 0.116 and a 95 percent confidence interval for $\epsilon$ of $[-0.363,0.091]$. The estimated $\epsilon$ is close to zero, consistent with the estimate of Roberts and Schlenker 2013 of -0.066 .

The last parameters to estimate are the demand shifters $A_{m}$. Given a particular $\sigma$, an associated estimated set of $\alpha_{i m}$, and a particular $\epsilon$, I estimate $A_{m}$ as an average across years:

$$
A_{m}=\frac{1}{T} \sum_{t} \frac{Q_{m t}}{N_{m t} P_{m t}^{\epsilon}}
$$

To compute standard errors, I implement a two-stage bootstrap procedure with 10,000 iterations in which I first re-estimate the set of $\alpha_{i m}$ and then the associated average $A_{m}$.

Table A8 reports estimates for $A_{m}$ and $\alpha_{i m}$ for 20 individual countries and 8 groups of countries. 
Different values of $A_{m}$ (a per-capita variable) may reflect several factors including the relative importance of cereal grains in local diets vis-à-vis other foods like tubers. Country groups were formed due to the limited number of annual observations. Countries with less than 7 observations were given special priority for group formation. Groups were formed from contiguous countries having the same set of grains and similar per-capita consumption of each grain. The maximum number of annual observations for a given country is 9 as trade data for 2013 was unavailable at the time of estimation. Even with the aggregation of some countries into groups, the number of annual observations per unit remains low. However, using multiple observations per country is an improvement over using a single year (the standard in the literature) and allows me to quantify the variation among observations.

Table A8: Demand Parameter Estimates for 28 Countries or Country Groups (1/2)

\begin{tabular}{|c|c|c|c|c|c|c|c|c|}
\hline & $A$ & $\alpha_{\text {maize }}$ & $\alpha_{\text {millet }}$ & $\alpha_{\text {rice }}$ & $\alpha_{\text {sorghum }}$ & $\alpha_{t e f f}$ & $\alpha_{\text {wheat }}$ & Observ. \\
\hline Benin & $\begin{array}{l}179.4 \\
(10.1)\end{array}$ & $\begin{array}{c}0.329 \\
(0.034)\end{array}$ & & $\begin{array}{c}0.614 \\
(0.037)\end{array}$ & $\begin{array}{c}0.058 \\
(0.004)\end{array}$ & & & 9 \\
\hline Burkina Faso & $\begin{array}{c}170.5 \\
(7.5)\end{array}$ & & $\begin{array}{c}0.421 \\
(0.009)\end{array}$ & & $\begin{array}{c}0.579 \\
(0.009)\end{array}$ & & & 7 \\
\hline Cameroon & $\begin{array}{l}90.7 \\
(3.2)\end{array}$ & $\begin{array}{c}0.618 \\
(0.015)\end{array}$ & & $\begin{array}{c}0.382 \\
(0.015)\end{array}$ & & & & 8 \\
\hline Chad & $\begin{array}{l}115.9 \\
(4.9)\end{array}$ & $\begin{array}{c}0.145 \\
(0.007)\end{array}$ & $\begin{array}{c}0.363 \\
(0.015)\end{array}$ & $\begin{array}{c}0.140 \\
(0.005)\end{array}$ & $\begin{array}{c}0.352 \\
(0.011)\end{array}$ & & & 9 \\
\hline Côte d'Ivoire & $\begin{array}{l}101.4 \\
(3.8)\end{array}$ & $\begin{array}{c}0.172 \\
(0.008)\end{array}$ & & $\begin{array}{c}0.828 \\
(0.008)\end{array}$ & & & & 7 \\
\hline Djibouti & $\begin{array}{l}45.0 \\
(5.5)\end{array}$ & & & $\begin{array}{c}1 \\
(0)\end{array}$ & & & & 9 \\
\hline Eritrea & $\begin{array}{c}38.3 \\
(11.0)\end{array}$ & & & & $\begin{array}{c}1 \\
(0)\end{array}$ & & & 3 \\
\hline Ethiopia & $\begin{array}{l}143.0 \\
(5.2)\end{array}$ & $\begin{array}{c}0.210 \\
(0.006)\end{array}$ & & & $\begin{array}{c}0.176 \\
(0.010)\end{array}$ & $\begin{array}{c}0.308 \\
(0.015)\end{array}$ & $\begin{array}{c}0.306 \\
(0.011)\end{array}$ & 7 \\
\hline Ghana & $\begin{array}{l}102.5 \\
(5.2)\end{array}$ & $\begin{array}{c}0.401 \\
(0.024)\end{array}$ & $\begin{array}{c}0.074 \\
(0.006)\end{array}$ & $\begin{array}{c}0.436 \\
(0.021)\end{array}$ & $\begin{array}{c}0.088 \\
(0.007)\end{array}$ & & & 7 \\
\hline Kenya & $\begin{array}{l}79.1 \\
(2.9)\end{array}$ & $\begin{array}{c}1 \\
(0)\end{array}$ & & & & & & 9 \\
\hline Malawi & $\begin{array}{l}169.0 \\
(14.7)\end{array}$ & $\begin{array}{c}1 \\
(0)\end{array}$ & & & & & & 9 \\
\hline Mali & $\begin{array}{l}234.7 \\
(13.2)\end{array}$ & & $\begin{array}{c}0.285 \\
(0.018)\end{array}$ & $\begin{array}{c}0.515 \\
(0.017)\end{array}$ & $\begin{array}{c}0.201 \\
(0.007)\end{array}$ & & & 7 \\
\hline
\end{tabular}


Table A8: Demand Parameter Estimates for 28 Countries or Country Groups (2/2)

\begin{tabular}{|c|c|c|c|c|c|c|c|c|}
\hline & $A$ & $\alpha_{\text {maize }}$ & $\alpha_{\text {millet }}$ & $\alpha_{\text {rice }}$ & $\alpha_{\text {sorghum }}$ & $\alpha_{t e f f}$ & $\alpha_{\text {wheat }}$ & Observ \\
\hline Mauritania & $\begin{array}{l}70.1 \\
(4.4)\end{array}$ & & & $\begin{array}{c}0.680 \\
(0.049)\end{array}$ & $\begin{array}{c}0.320 \\
(0.049)\end{array}$ & & & 5 \\
\hline Mozambique & $\begin{array}{l}70.0 \\
(4.2)\end{array}$ & $\begin{array}{c}0.602 \\
(0.027)\end{array}$ & & $\begin{array}{c}0.398 \\
(0.027)\end{array}$ & & & & 8 \\
\hline Niger & $\begin{array}{l}251.2 \\
(11.8)\end{array}$ & & $\begin{array}{c}0.766 \\
(0.006)\end{array}$ & & $\begin{array}{c}0.234 \\
(0.006)\end{array}$ & & & 9 \\
\hline Nigeria & $\begin{array}{c}166.9 \\
(7.4)\end{array}$ & $\begin{array}{l}0.218 \\
(0.011)\end{array}$ & $\begin{array}{c}0.257 \\
(0.009)\end{array}$ & $\begin{array}{c}0.223 \\
(0.013)\end{array}$ & $\begin{array}{c}0.302 \\
(0.015)\end{array}$ & & & 7 \\
\hline Somalia & $\begin{array}{l}29.6 \\
(2.6)\end{array}$ & $\begin{array}{c}0.590 \\
(0.033)\end{array}$ & & & $\begin{array}{c}0.410 \\
(0.033)\end{array}$ & & & 9 \\
\hline Tanzania & $\begin{array}{l}98.1 \\
(5.7)\end{array}$ & $\begin{array}{l}0.561 \\
(0.025)\end{array}$ & & $\begin{array}{c}0.439 \\
(0.025)\end{array}$ & & & & 7 \\
\hline Togo & $\begin{array}{c}158.9 \\
(3.3)\end{array}$ & $\begin{array}{c}0.400 \\
(0.013)\end{array}$ & & $\begin{array}{c}0.406 \\
(0.011)\end{array}$ & $\begin{array}{c}0.194 \\
(0.008)\end{array}$ & & & 9 \\
\hline Uganda & $\begin{array}{l}72.1 \\
(0.7)\end{array}$ & $\begin{array}{c}0.833 \\
(0.002)\end{array}$ & & & $\begin{array}{c}0.167 \\
(0.002)\end{array}$ & & & 2 \\
\hline $\begin{array}{l}\text { Angola/Botswana/ } \\
\text { Namibia }\end{array}$ & $\begin{array}{c}48.8 \\
(10.2)\end{array}$ & $\begin{array}{c}1 \\
(0)\end{array}$ & & & & & & 9 \\
\hline Burundi/Rwanda & $\begin{array}{l}31.6 \\
(3.7)\end{array}$ & $\begin{array}{c}0.627 \\
(0.026)\end{array}$ & & & $\begin{array}{c}0.373 \\
(0.026)\end{array}$ & & & 12 \\
\hline C.A.R./D.R. Congo & $\begin{array}{l}32.1 \\
(2.1)\end{array}$ & $\begin{array}{c}0.712 \\
(0.018)\end{array}$ & & $\begin{array}{c}0.288 \\
(0.018)\end{array}$ & & & & 14 \\
\hline Congo/Gabon & $\begin{array}{l}40.6 \\
(1.7)\end{array}$ & & & $\begin{array}{c}1 \\
(0)\end{array}$ & & & & 8 \\
\hline $\begin{array}{l}\text { Gambia/G.Bissau/ } \\
\text { Senegal }\end{array}$ & $\begin{array}{c}170.6 \\
(9.0)\end{array}$ & $\begin{array}{c}0.125 \\
(0.011)\end{array}$ & $\begin{array}{c}0.229 \\
(0.024)\end{array}$ & $\begin{array}{c}0.570 \\
(0.030)\end{array}$ & $\begin{array}{c}0.076 \\
(0.006)\end{array}$ & & & 19 \\
\hline $\begin{array}{l}\text { Guinea/Liberia/ } \\
\text { Sierra Leone }\end{array}$ & $\begin{array}{l}108.3 \\
(5.4)\end{array}$ & & & $\begin{array}{c}1 \\
(0)\end{array}$ & & & & 17 \\
\hline $\begin{array}{l}\text { Lesotho/Swaziland/ } \\
\text { Zambia/Zimbabwe }\end{array}$ & $\begin{array}{l}105.5 \\
(7.8)\end{array}$ & $\begin{array}{c}1 \\
(0)\end{array}$ & & & & & & 16 \\
\hline South Sudan/Sudan & $\begin{array}{l}212.7 \\
(10.1)\end{array}$ & $\begin{array}{c}0.018 \\
(0.005)\end{array}$ & $\begin{array}{c}0.089 \\
(0.008)\end{array}$ & & $\begin{array}{c}0.465 \\
(0.026)\end{array}$ & & $\begin{array}{c}0.428 \\
(0.033)\end{array}$ & 5 \\
\hline
\end{tabular}




\section{A6 Estimation: External Variation and Standard Errors}

The main paper reports standard errors using resampled data from within the sets $\mathbb{S}$ and $\mathbb{T}$ associated with the fixed point obtained when starting from my preferred initial guess $\mathbb{C}^{0}$. I also assessed the external variation in my parameter estimates and results by re-estimating the parameters starting from 20 different smaller initial guesses $\mathbb{C}^{0}$. These included initial guesses with trade costs set at $\$ 0.01 / \mathrm{t}-\mathrm{km}, \$ 0.02 / \mathrm{t}-\mathrm{km}$, and $\$ 0.04 / \mathrm{t}-\mathrm{km}$ (with $r_{m}$ and $k_{m}$ set at $0.001,0.002$, and 0.004 respectively), with all parameters set to one-half and one-quarter of my preferred initial guess, and 15 initial guesses in which each parameter was drawn from a uniform distribution from 0 up to its value in my preferred initial guess. Each initial guess converged to a distinct fixed point, taking between 11 and 22 iterations. I then solved for equilibrium using the fixed point's associated parameters under both newly-estimated high and counterfactual low trade costs. The number of initial guesses was limited to 20 due to the extremely long run-time involved (one month per initial guess). External standard errors reported below were obtained by taking the standard deviation of the values from the 20 different initial guesses.

Table A9 compares the external standard errors for the storage cost parameters to the point estimates and internal standard errors reported in table 3 of the main paper. All external standard errors are substantially smaller than the associated internal standard errors. All storage cost parameters obtained from the 20 different initial guesses are well within 95 percent confidence intervals constructed around the initial point estimates using the internal standard errors. External standard errors for the trade cost parameters are presented alongside internal standard errors in the following section.

Table A10 compares the external standard errors for the aggregate results from the main counterfactual to the point estimates and internal standard errors reported in table 8 of the main paper. Again, all external standard errors are substantially smaller than the associated internal standard errors, and all results obtained from the 20 different initial guesses are well within 95 percent confidence intervals constructed around the initial point estimates using the internal standard errors. 
Table A9: Comparison of Standard Errors for Storage Cost Parameters

\begin{tabular}{lcccc}
\hline Region & Parameter & Pt. Estimate & Internal SE & External SE \\
\hline Southern & $r_{m}$ & 0.0269 & $(0.0082)$ & $(0.0020)$ \\
East/Horn & $r_{m}$ & 0.0281 & $(0.0034)$ & $(0.0012)$ \\
Central & $r_{m}$ & 0.0404 & $(0.0150)$ & $(0.0025)$ \\
West Coast & $r_{m}$ & 0.0174 & $(0.0029)$ & $(0.0007)$ \\
Sahel & $r_{m}$ & 0.0259 & $(0.0068)$ & $(0.0013)$ \\
\hline Southern & $k_{m}$ & 0.0069 & $(0.0020)$ & $(0.0005)$ \\
East/Horn & $k_{m}$ & 0.0061 & $(0.0011)$ & $(0.0002)$ \\
Central & $k_{m}$ & 0.0218 & $(0.0099)$ & $(0.0006)$ \\
West Coast & $k_{m}$ & 0.0122 & $(0.0013)$ & $(0.0004)$ \\
Sahel & $k_{m}$ & 0.0050 & $(0.0023)$ & $(0.0006)$ \\
\hline
\end{tabular}

Table A10: Comparison of Standard Errors for Aggregate Results

\begin{tabular}{lcccccc}
\hline & Quantity & Internal SE & External SE & Percent & Internal SE & External SE \\
\hline Avg. Grain Price Index & $-\$ 0.25 / \mathrm{kg}$ & $(\$ 0.01 / \mathrm{kg})$ & $(\$ 0.003 / \mathrm{kg})$ & -46.4 & $(1.0)$ & $(0.3)$ \\
Net Ag. Revenues & $-\$ 117.4 \mathrm{bill}$ & $(\$ 10.2 \mathrm{bill})$ & $(\$ 3.8 \mathrm{bill})$ & -42.1 & $(1.7)$ & $(0.7)$ \\
Expend. on Grains & $-\$ 226.4 \mathrm{bill}$ & $(\$ 14.1 \mathrm{bill})$ & $(\$ 4.7 \mathrm{bill})$ & -44.1 & $(1.0)$ & $(0.5)$ \\
Net Grain Imports & +32.0 mill t & $(4.6$ mill t $)$ & $(1.7$ mill t $)$ & +17.5 & $(1.8)$ & $(1.1)$ \\
Trade Cost Expend. & $-\$ 65.5$ bill & $(\$ 3.0 \mathrm{bill})$ & $(\$ 1.0 \mathrm{bill})$ & -72.8 & $(1.0)$ & $(0.4)$ \\
Storage Cost Expend. & $-\$ 27.3$ bill & $(\$ 1.6 \mathrm{bill})$ & $(\$ 0.5 \mathrm{bill})$ & -41.0 & $(2.0)$ & $(0.5)$ \\
Outside Good Cons'n & $+\$ 109.0$ bill & $(\$ 4.7 \mathrm{bill})$ & $(\$ 1.1 \mathrm{bill})$ & +2.08 & $(0.097)$ & $(0.023)$ \\
Welfare $(\mathrm{EV})$ & $+\$ 125.0 \mathrm{bill})$ & $(\$ 5.6 \mathrm{bill})$ & $(\$ 1.4 \mathrm{bill})$ & +2.17 & $(0.097)$ & $(0.024)$ \\
\hline
\end{tabular}




\section{A7 Estimation: Trade Cost Parameters}

Table A11, which begins on the next page, shows estimated trade costs $\tau_{m n}$ for each of the 413 overland links in my model. Each link is only listed once, i.e. a link with a given "Market 1" is only listed if its "Market 2" has not yet been listed as a "Market 1". Links are listed by country of "Market 1" (both domestic and overland international links are included). Distances are in kilometers, with cost per tonne-kilometer also reported. Observations listed are observations with trade on the last iteration in which trade costs for that particular link were estimated prior to convergence. Internal standard errors were obtained by bootstrapping (10,000 iterations) using this set of final-iteration observations for resampling with replacement. External standard errors were obtained using the 20 different initial guesses described in the previous section. Unlike the storage cost parameters, a substantial portion of the trade cost parameters (41\%) have external standard errors that exceed their internal standard errors. However, as was shown in Table A10, the external standard errors for the aggregate counterfactual results are all much smaller than the associated internal standard errors.

Table A12, which follows Table A11, shows estimated trade costs $\tau_{m n}$ for the 47 links between 30 African ports and the world market (Bangkok for rice and the US Gulf for maize, sorghum, and wheat). Whether or not a port is linked to Bangkok and/or the US Gulf depends on its mix of crops. Observations and standard errors were obtained using the same procedure as for table A11. Over 70 percent of links have costs between $\$ 0.10$ and $\$ 0.50 / \mathrm{kg}(\$ 100-\$ 500 /$ tonne). The lowest cost ports $(\$ 70-\$ 100 /$ tonne) are Nacala (Mozambique), Mombasa (Kenya), Mogadishu (Somalia), and Dakar (Senegal) while the highest cost ports ( $>\$ 1000 /$ tonne) are Luanda (Angola), Massawa (Eritrea), and Bissau (Guinea-Bissau).

Table A13, which follows Table A12, shows correlations between these port-to-world-market trade costs and port characteristics. Although none of the correlations are significant at the 5 percent level (which is likely due to the small sample size and the idiosyncratic nature of port costs), most of the point estimates have the expected signs, with higher costs correlated with smaller port populations, lower port volumes, lower Corruption Perception Indices, and higher import tariffs. Import tariffs were obtained for the relevant grains from the World Bank's World

Integrated Trade Solution (WITS). "High Volume" is an indicator variable for whether the port handled more than 500,000 TEUs in 2007 (African Development Bank 2010). 
Table A11: Estimated Overland Trade Costs (1/10)

\begin{tabular}{|c|c|c|c|c|c|c|c|c|}
\hline & Market 1 & Market 2 & $\tau_{m n}$ & Intern. SE & Extern. SE & Observ. & Distance & $/ \mathrm{t}-\mathrm{km}$ \\
\hline Botswana & Gaborone & Johannesburg & 0.1142 & $(0.0076)$ & $(0.0000)$ & 52 & 366 & 0.3120 \\
\hline Lesotho & Maseru & Johannesburg & 0.0707 & $(0.0087)$ & $(0.0000)$ & 31 & 420 & 0.1683 \\
\hline \multirow[t]{12}{*}{ Malawi } & Blantyre & Lilongwe & 0.0536 & $(0.0236)$ & $(0.0149)$ & 2 & 365 & 0.1468 \\
\hline & Blantyre & Mangochi & 0.0601 & $(0.0137)$ & $(0.0095)$ & 17 & 191 & 0.3147 \\
\hline & Blantyre & Milange & 0.0883 & $(0.0185)$ & $(0.0129)$ & 14 & 115 & 0.7682 \\
\hline & Blantyre & Tete & 0.0609 & $(0.0227)$ & $(0.0266)$ & 4 & 215 & 0.2835 \\
\hline & Karonga & Mzuzu & 0.0530 & $(0.0088)$ & $(0.0028)$ & 21 & 222 & 0.2389 \\
\hline & Karonga & Mbeya & 0.1131 & $(0.0359)$ & $(0.0090)$ & 5 & 161 & 0.7023 \\
\hline & Lilongwe & Mangochi & 0.0558 & $(0.0097)$ & $(0.0111)$ & 5 & 272 & 0.2053 \\
\hline & Lilongwe & Mzuzu & 0.0323 & $(0.0022)$ & $(0.0055)$ & 92 & 358 & 0.0901 \\
\hline & Lilongwe & Tete & 0.0533 & $(0.0079)$ & $(0.0072)$ & 15 & 370 & 0.1441 \\
\hline & Lilongwe & Chipata & 0.0644 & $(0.0044)$ & $(0.0156)$ & 95 & 145 & 0.4441 \\
\hline & Mangochi & Cuamba & 0.0804 & $(0.0049)$ & $(0.0072)$ & 40 & 205 & 0.3924 \\
\hline & Mangochi & Lichinga & 0.0665 & $(0.0139)$ & $(0.0079)$ & 14 & 221 & 0.3008 \\
\hline \multirow[t]{19}{*}{ Mozamb. } & Beira & Chimoio & 0.0989 & $(0.0132)$ & $(0.0124)$ & 36 & 204 & 0.4848 \\
\hline & Beira & Quelimane & 0.0424 & $(0.0100)$ & $(0.0072)$ & 12 & 487 & 0.0871 \\
\hline & Chimoio & Maxixe & 0.1286 & $(0.0084)$ & $(0.0215)$ & 90 & 677 & 0.1900 \\
\hline & Chimoio & Quelimane & 0.1516 & $(0.0179)$ & $(0.0159)$ & 20 & 552 & 0.2747 \\
\hline & Chimoio & Tete & 0.1919 & $(0.0272)$ & $(0.0009)$ & 44 & 381 & 0.5036 \\
\hline & Chimoio & Mutare & 0.0597 & $(0.0210)$ & $(0.0133)$ & 3 & 96 & 0.6218 \\
\hline & Cuamba & Lichinga & 0.3503 & $(0.0528)$ & $(0.0400)$ & 9 & 315 & 1.1119 \\
\hline & Cuamba & Nampula & 0.1566 & $(0.0242)$ & $(0.0180)$ & 46 & 358 & 0.4374 \\
\hline & Maputo & XaiXai & 0.1047 & $(0.0112)$ & $(0.0035)$ & 34 & 216 & 0.4847 \\
\hline & Maputo & Mbabane & 0.0997 & $(0.0117)$ & $(0.0146)$ & 24 & 221 & 0.4510 \\
\hline & Maputo & Johannesburg & 0.2131 & $(0.0115)$ & $(0.0098)$ & 38 & 545 & 0.3910 \\
\hline & Maxixe & XaiXai & 0.0738 & $(0.0111)$ & $(0.0075)$ & 30 & 256 & 0.2883 \\
\hline & Milange & Quelimane & 0.0833 & $(0.0159)$ & $(0.0223)$ & 19 & 320 & 0.2603 \\
\hline & Nacala & Nampula & 0.0744 & $(0.0141)$ & $(0.0102)$ & 27 & 192 & 0.3875 \\
\hline & Nampula & Pemba & 0.0926 & $(0.0067)$ & $(0.0022)$ & 79 & 404 & 0.2293 \\
\hline & Nampula & Quelimane & 0.0488 & $(0.0041)$ & $(0.0119)$ & 76 & 551 & 0.0885 \\
\hline & Pemba & Mtwara & 0.1822 & $(0.0341)$ & $(0.0270)$ & 18 & 445 & 0.4095 \\
\hline & Tete & Chipata & 0.1590 & $(0.0213)$ & $(0.0194)$ & 9 & 379 & 0.4196 \\
\hline & Tete & Harare & 0.0324 & $(0.0075)$ & $(0.0025)$ & 14 & 383 & 0.0845 \\
\hline \multirow[t]{7}{*}{ Namibia } & KatimaMulilo & Oshakati & 0.1634 & $(0.0137)$ & $(0.0150)$ & 14 & 1114 & 0.1467 \\
\hline & KatimaMulilo & Windhoek & 0.1026 & $(0.0228)$ & $(0.0093)$ & 12 & 1226 & 0.0837 \\
\hline & KatimaMulilo & Livingstone & 0.2864 & $(0.0345)$ & $(0.0098)$ & 10 & 208 & 1.3772 \\
\hline & KatimaMulilo & Mongu & 0.3367 & $(0.0174)$ & $(0.0077)$ & 15 & 312 & 1.0792 \\
\hline & Oshakati & Windhoek & 0.0902 & $(0.0118)$ & $(0.0076)$ & 14 & 716 & 0.1259 \\
\hline & Swakopmund & Windhoek & 0.0279 & $(0.0040)$ & $(0.0006)$ & 16 & 362 & 0.0771 \\
\hline & Windhoek & Johannesburg & 0.2600 & $(0.0058)$ & $(0.0052)$ & 16 & 1365 & 0.1905 \\
\hline Swaziland & Mbabane & Johannesburg & 0.0894 & $(0.0062)$ & $(0.0104)$ & 89 & 357 & 0.2505 \\
\hline
\end{tabular}


Table A11: Estimated Overland Trade Costs (2/10)

\begin{tabular}{|c|c|c|c|c|c|c|c|c|}
\hline & Market 1 & Market 2 & $\tau_{m n}$ & Intern. SE & Extern. SE & Observ. & Distance & $/ \mathrm{t}-\mathrm{km}$ \\
\hline \multirow[t]{16}{*}{ Zambia } & Chipata & Lusaka & 0.0368 & $(0.0044)$ & $(0.0023)$ & 46 & 572 & 0.0644 \\
\hline & Kabwe & Kasama & 0.1438 & $(0.0325)$ & $(0.0142)$ & 4 & 721 & 0.1994 \\
\hline & Kabwe & Kitwe & 0.0425 & $(0.0023)$ & $(0.0005)$ & 119 & 221 & 0.1922 \\
\hline & Kabwe & Lusaka & 0.0571 & $(0.0068)$ & $(0.0027)$ & 24 & 140 & 0.4081 \\
\hline & Kabwe & Mbeya & 0.0921 & $(0.0228)$ & $(0.0082)$ & 14 & 986 & 0.0934 \\
\hline & Kasama & Mbeya & 0.0935 & $(0.0142)$ & $(0.0049)$ & 11 & 400 & 0.2337 \\
\hline & Kasama & Sumbawanga & 0.0902 & $(0.0113)$ & $(0.0105)$ & 17 & 287 & 0.3144 \\
\hline & Kitwe & Solwezi & 0.0362 & $(0.0059)$ & $(0.0054)$ & 22 & 223 & 0.1625 \\
\hline & Kitwe & Lubumbashi & 0.8285 & $(0.3733)$ & $(0.1650)$ & 5 & 187 & 4.4306 \\
\hline & Livingstone & Lusaka & 0.0323 & $(0.0041)$ & $(0.0062)$ & 27 & 477 & 0.0678 \\
\hline & Livingstone & Mongu & 0.0854 & $(0.0165)$ & $(0.0015)$ & 10 & 508 & 0.1680 \\
\hline & Livingstone & Hwange & 0.1884 & $(0.0110)$ & $(0.0003)$ & 9 & 117 & 1.6103 \\
\hline & Lusaka & Mongu & 0.0662 & $(0.0145)$ & $(0.0086)$ & 9 & 607 & 0.1091 \\
\hline & Lusaka & Harare & 0.0807 & $(0.0092)$ & $(0.0184)$ & 24 & 493 & 0.1636 \\
\hline & Mongu & Solwezi & 0.1750 & $(0.0106)$ & $(0.0159)$ & 3 & 591 & 0.2961 \\
\hline & Solwezi & Lubumbashi & 0.4837 & $(0.0522)$ & $(0.0415)$ & 60 & 166 & 2.9139 \\
\hline \multirow{8}{*}{ Zimbabwe } & Bulawayo & Harare & 0.0344 & $(0.0081)$ & $(0.0065)$ & 32 & 447 & 0.0769 \\
\hline & Bulawayo & Hwange & 0.0422 & $(0.0094)$ & $(0.0039)$ & 9 & 339 & 0.1245 \\
\hline & Bulawayo & Masvingo & 0.1175 & $(0.0065)$ & $(0.0143)$ & 4 & 283 & 0.4152 \\
\hline & Bulawayo & Johannesburg & 0.0805 & $(0.0214)$ & $(0.0345)$ & 10 & 863 & 0.0933 \\
\hline & Harare & Masvingo & 0.0485 & $(0.0033)$ & $(0.0087)$ & 39 & 294 & 0.1648 \\
\hline & Harare & Mutare & 0.0155 & $(0.0095)$ & $(0.0042)$ & 11 & 254 & 0.0608 \\
\hline & Masvingo & Mutare & 0.0500 & $(0.0082)$ & $(0.0018)$ & 3 & 298 & 0.1678 \\
\hline & Masvingo & Johannesburg & 0.1110 & $(0.0061)$ & $(0.0162)$ & 40 & 827 & 0.1342 \\
\hline \multirow{2}{*}{ Angola } & Luanda & Kinshasa & 1.0407 & $(0.0187)$ & $(0.0795)$ & 4 & 531 & 1.9599 \\
\hline & Luanda & Matadi & 1.0745 & $(0.0667)$ & $(0.0166)$ & 4 & 920 & 1.1679 \\
\hline \multirow[t]{8}{*}{ Burundi } & Bujumbura & Gitega & 0.0706 & $(0.0034)$ & $(0.0074)$ & 184 & 101 & 0.6986 \\
\hline & Bujumbura & Uvira & 0.1002 & $(0.0060)$ & $(0.0088)$ & 60 & 31 & 3.2335 \\
\hline & Bujumbura & Butare & 0.1755 & $(0.0554)$ & $(0.0255)$ & 7 & 162 & 1.0834 \\
\hline & Bujumbura & Kigoma & 0.1225 & $(0.0090)$ & $(0.0023)$ & 34 & 229 & 0.5350 \\
\hline & Gitega & Muyinga & 0.0842 & $(0.0031)$ & $(0.0093)$ & 183 & 96 & 0.8773 \\
\hline & Gitega & Kigoma & 0.0847 & $(0.0192)$ & $(0.0068)$ & 18 & 218 & 0.3885 \\
\hline & Muyinga & Butare & 0.0811 & $(0.0039)$ & $(0.0044)$ & 108 & 118 & 0.6871 \\
\hline & Muyinga & Mwanza & 0.1016 & $(0.0134)$ & $(0.0053)$ & 33 & 423 & 0.2402 \\
\hline \multirow[t]{6}{*}{ C.A.R. } & Bambari & Bangassou & 0.3381 & $(0.0386)$ & $(0.1362)$ & 48 & 353 & 0.9577 \\
\hline & Bambari & Bangui & 0.6447 & $(0.0457)$ & $(0.0224)$ & 14 & 385 & 1.6744 \\
\hline & Bambari & Gbadolite & 0.4744 & $(0.0806)$ & $(0.1192)$ & 23 & 250 & 1.8975 \\
\hline & Bangassou & Gbadolite & 0.4891 & $(0.0863)$ & $(0.0309)$ & 11 & 290 & 1.6866 \\
\hline & Bangui & Impfondo & 0.1576 & $(0.0259)$ & $(0.0065)$ & 5 & 320 & 0.4924 \\
\hline & Bangui & Zongo & 0.4158 & $(0.0895)$ & $(0.0269)$ & 4 & 2 & 207.8856 \\
\hline \multirow[t]{4}{*}{ Congo } & Brazzaville & Impfondo & 0.2016 & $(0.0489)$ & $(0.0222)$ & 9 & 780 & 0.2584 \\
\hline & Brazzaville & PointeNoire & 0.1169 & $(0.0302)$ & $(0.0152)$ & 10 & 548 & 0.2133 \\
\hline & Brazzaville & Kinshasa & 0.3888 & $(0.0602)$ & $(0.0396)$ & 14 & 4 & 97.2042 \\
\hline & Impfondo & Mbandaka & 0.4169 & $(0.0229)$ & $(0.0205)$ & 10 & 352 & 1.1845 \\
\hline
\end{tabular}


Table A11: Estimated Overland Trade Costs (3/10)

\begin{tabular}{|c|c|c|c|c|c|c|c|c|}
\hline & Market 1 & Market 2 & $\tau_{m n}$ & Intern. SE & Extern. SE & Observ. & Distance & $/ \mathrm{t}-\mathrm{km}$ \\
\hline \multirow[t]{41}{*}{ D.R. Congo } & Bandundu & Kikwit & 0.3097 & $(0.0610)$ & $(0.0871)$ & 18 & 395 & 0.7841 \\
\hline & Bandundu & Kinshasa & 0.3963 & $(0.0584)$ & $(0.0239)$ & 18 & 395 & 1.0033 \\
\hline & Bandundu & Mbandaka & 0.4737 & $(0.0712)$ & $(0.1274)$ & 20 & 580 & 0.8167 \\
\hline & Bukavu & Goma & 0.2805 & $(0.0366)$ & $(0.0058)$ & 42 & 199 & 1.4097 \\
\hline & Bukavu & Kindu & 0.4623 & $(0.0291)$ & $(0.1188)$ & 119 & 695 & 0.6652 \\
\hline & Bukavu & Kisangani & 0.3585 & $(0.0137)$ & $(0.0507)$ & 119 & 641 & 0.5593 \\
\hline & Bukavu & Uvira & 0.3384 & $(0.0388)$ & $(0.0320)$ & 41 & 137 & 2.4700 \\
\hline & Bukavu & Butare & 0.1966 & $(0.0163)$ & $(0.0058)$ & 48 & 147 & 1.3375 \\
\hline & Bunia & Butembo & 0.3463 & $(0.0559)$ & $(0.0344)$ & 27 & 251 & 1.3798 \\
\hline & Bunia & Isiro & 0.2737 & $(0.0321)$ & $(0.0027)$ & 40 & 486 & 0.5632 \\
\hline & Bunia & Kisangani & 0.3564 & $(0.0127)$ & $(0.0673)$ & 120 & 706 & 0.5049 \\
\hline & Bunia & Juba & 0.2254 & $(0.0131)$ & $(0.0143)$ & 59 & 633 & 0.3560 \\
\hline & Bunia & Arua & 0.2819 & $(0.0170)$ & $(0.0264)$ & 5 & 256 & 1.1013 \\
\hline & Bunia & Gulu & 0.3151 & $(0.0386)$ & $(0.0094)$ & 13 & 350 & 0.9004 \\
\hline & Butembo & Goma & 0.3609 & $(0.0933)$ & $(0.0316)$ & 8 & 313 & 1.1531 \\
\hline & Butembo & Mbarara & 0.1645 & $(0.0109)$ & $(0.0104)$ & 25 & 303 & 0.5430 \\
\hline & Gbadolite & Mbandaka & 0.5745 & $(0.0806)$ & $(0.1134)$ & 2 & 675 & 0.8511 \\
\hline & Gbadolite & Zongo & 0.5942 & $(0.1088)$ & $(0.2071)$ & 2 & 404 & 1.4708 \\
\hline & Goma & Gisenyi & 0.1481 & $(0.0239)$ & $(0.0019)$ & 35 & 9 & 16.4538 \\
\hline & Isiro & Kisangani & 0.4951 & $(0.1192)$ & $(0.0507)$ & 9 & 577 & 0.8581 \\
\hline & Isiro & Juba & 0.2966 & $(0.0179)$ & $(0.0417)$ & 43 & 674 & 0.4401 \\
\hline & Kalemie & Kamina & 0.3238 & $(0.0379)$ & $(0.0061)$ & 40 & 990 & 0.3271 \\
\hline & Kalemie & Kindu & 0.4430 & $(0.0325)$ & $(0.0667)$ & 120 & 802 & 0.5524 \\
\hline & Kalemie & Uvira & 0.7118 & $(0.2536)$ & $(0.0940)$ & 2 & 312 & 2.2814 \\
\hline & Kalemie & Kigoma & 0.3550 & $(0.0467)$ & $(0.0089)$ & 31 & 148 & 2.3986 \\
\hline & Kamina & Kolwezi & 0.3372 & $(0.0407)$ & $(0.0035)$ & 37 & 512 & 0.6586 \\
\hline & Kamina & Lubumbashi & 0.2104 & $(0.0305)$ & $(0.0125)$ & 42 & 578 & 0.3640 \\
\hline & Kamina & MbujiMayi & 0.2612 & $(0.0353)$ & $(0.0165)$ & 35 & 458 & 0.5702 \\
\hline & Kananga & Kisangani & 0.3761 & $(0.0399)$ & $(0.1105)$ & 120 & 1282 & 0.2934 \\
\hline & Kananga & MbujiMayi & 0.5579 & $(0.2753)$ & $(0.1817)$ & 6 & 179 & 3.1169 \\
\hline & Kananga & Tshikapa & 0.7875 & $(0.2223)$ & $(0.1986)$ & 5 & 236 & 3.3371 \\
\hline & Kikwit & Kinshasa & 0.3412 & $(0.0404)$ & $(0.0211)$ & 42 & 347 & 0.9832 \\
\hline & Kikwit & Tshikapa & 0.4704 & $(0.0275)$ & $(0.0473)$ & 118 & 519 & 0.9064 \\
\hline & Kindu & Kisangani & 0.4090 & $(0.1099)$ & $(0.0281)$ & 34 & 592 & 0.6910 \\
\hline & Kindu & MbujiMayi & 0.7201 & $(0.1357)$ & $(0.0422)$ & 8 & 752 & 0.9576 \\
\hline & Kinshasa & Matadi & 0.3663 & $(0.0257)$ & $(0.0875)$ & 117 & 362 & 1.0119 \\
\hline & Kinshasa & Mbandaka & 0.3287 & $(0.0154)$ & $(0.0557)$ & 120 & 620 & 0.5302 \\
\hline & Kisangani & Mbandaka & 0.1624 & $(0.0187)$ & $(0.0025)$ & 89 & 976 & 0.1664 \\
\hline & Kolwezi & Lubumbashi & 0.2304 & $(0.0167)$ & $(0.0404)$ & 112 & 304 & 0.7578 \\
\hline & Mbandaka & Zongo & 0.2274 & $(0.0318)$ & $(0.0057)$ & 55 & 672 & 0.3384 \\
\hline & Uvira & Kigoma & 0.1787 & $(0.0165)$ & $(0.0072)$ & 54 & 172 & 1.0390 \\
\hline
\end{tabular}


Table A11: Estimated Overland Trade Costs (4/10)

\begin{tabular}{|c|c|c|c|c|c|c|c|c|}
\hline & Market 1 & Market 2 & $\tau_{m n}$ & Intern. SE & Extern. SE & Observ. & Distance & $/ \mathrm{t}-\mathrm{km}$ \\
\hline \multirow[t]{21}{*}{ Kenya } & Eldoret & Kisumu & 0.0508 & $(0.0156)$ & $(0.0057)$ & 10 & 117 & 0.4342 \\
\hline & Eldoret & Lodwar & 0.2718 & $(0.0126)$ & $(0.0007)$ & 109 & 367 & 0.7406 \\
\hline & Eldoret & Nakuru & 0.0224 & $(0.0017)$ & $(0.0012)$ & 64 & 155 & 0.1444 \\
\hline & Eldoret & Jinja & 0.0693 & $(0.0054)$ & $(0.0144)$ & 25 & 268 & 0.2585 \\
\hline & Garissa & Mombasa & 0.1514 & $(0.0117)$ & $(0.0205)$ & 103 & 463 & 0.3269 \\
\hline & Garissa & Nairobi & 0.2024 & $(0.0201)$ & $(0.0231)$ & 36 & 366 & 0.5529 \\
\hline & Garissa & Wajir & 0.1552 & $(0.0069)$ & $(0.0383)$ & 115 & 323 & 0.4804 \\
\hline & Garissa & Kismayo & 0.2042 & $(0.0055)$ & $(0.0105)$ & 4 & 410 & 0.4981 \\
\hline & Kisumu & Nakuru & 0.1075 & $(0.0207)$ & $(0.0146)$ & 5 & 183 & 0.5877 \\
\hline & Kisumu & Musoma & 0.0743 & $(0.0046)$ & $(0.0209)$ & 82 & 307 & 0.2421 \\
\hline & Kisumu & Jinja & 0.1623 & $(0.0054)$ & $(0.0204)$ & 25 & 238 & 0.6821 \\
\hline & Lodwar & Juba & 0.1741 & $(0.0197)$ & $(0.0476)$ & 50 & 641 & 0.2716 \\
\hline & Mandera & Wajir & 0.1687 & $(0.0237)$ & $(0.0121)$ & 11 & 420 & 0.4016 \\
\hline & Mandera & Baidoa & 0.2629 & $(0.0125)$ & $(0.0344)$ & 109 & 269 & 0.9773 \\
\hline & Mandera & Awasa & 0.1598 & $(0.0171)$ & $(0.0140)$ & 31 & 704 & 0.2270 \\
\hline & Mombasa & Nairobi & 0.0246 & $(0.0014)$ & $(0.0006)$ & 108 & 500 & 0.0493 \\
\hline & Mombasa & Tanga & 0.1103 & $(0.0138)$ & $(0.0107)$ & 14 & 164 & 0.6727 \\
\hline & Moyale & Wajir & 0.4087 & $(0.0287)$ & $(0.0792)$ & 2 & 258 & 1.5839 \\
\hline & Moyale & Yabelo & 0.0979 & $(0.0083)$ & $(0.0000)$ & 67 & 213 & 0.4594 \\
\hline & Nairobi & Nakuru & 0.0388 & $(0.0033)$ & $(0.0009)$ & 39 & 159 & 0.2439 \\
\hline & Nairobi & Arusha & 0.1556 & $(0.0003)$ & $(0.0083)$ & 2 & 269 & 0.5785 \\
\hline \multirow[t]{6}{*}{ Rwanda } & Butare & Gisenyi & 0.0655 & $(0.0238)$ & $(0.0033)$ & 2 & 202 & 0.3240 \\
\hline & Butare & Kigali & 0.0972 & $(0.0144)$ & $(0.0037)$ & 23 & 123 & 0.7906 \\
\hline & Gisenyi & Kigali & 0.0905 & $(0.0082)$ & $(0.0012)$ & 49 & 151 & 0.5996 \\
\hline & Gisenyi & Mbarara & 0.0785 & $(0.0115)$ & $(0.0174)$ & 7 & 299 & 0.2625 \\
\hline & Kigali & Mwanza & 0.0786 & $(0.0329)$ & $(0.0237)$ & 11 & 533 & 0.1474 \\
\hline & Kigali & Mbarara & 0.1409 & $(0.0342)$ & $(0.0085)$ & 10 & 242 & 0.5820 \\
\hline \multirow{11}{*}{ Somalia } & Baidoa & Mogadishu & 0.0604 & $(0.0037)$ & $(0.0010)$ & 112 & 247 & 0.2447 \\
\hline & Baidoa & Awasa & 0.3026 & $(0.0446)$ & $(0.0065)$ & 6 & 891 & 0.3396 \\
\hline & Beledweyne & Galkayo & 0.1293 & $(0.0062)$ & $(0.0043)$ & 176 & 389 & 0.3325 \\
\hline & Beledweyne & Mogadishu & 0.0494 & $(0.0024)$ & $(0.0005)$ & 182 & 339 & 0.1459 \\
\hline & Beledweyne & Gode & 0.0903 & $(0.0088)$ & $(0.0025)$ & 101 & 263 & 0.3432 \\
\hline & Beledweyne & Jijiga & 0.1001 & $(0.0052)$ & $(0.0156)$ & 118 & 599 & 0.1672 \\
\hline & Bosaso & Garoowe & 0.1210 & $(0.0065)$ & $(0.0043)$ & 191 & 443 & 0.2731 \\
\hline & Galkayo & Garoowe & 0.4358 & $(0.0728)$ & $(0.0852)$ & 8 & 230 & 1.8947 \\
\hline & Garoowe & Hargeisa & 0.3701 & $(0.0478)$ & $(0.0708)$ & 5 & 594 & 0.6230 \\
\hline & Hargeisa & Jijiga & 0.1663 & $(0.0065)$ & $(0.0016)$ & 149 & 165 & 1.0081 \\
\hline & Kismayo & Mogadishu & 0.0698 & $(0.0203)$ & $(0.0095)$ & 10 & 485 & 0.1438 \\
\hline
\end{tabular}


Table A11: Estimated Overland Trade Costs (5/10)

\begin{tabular}{|c|c|c|c|c|c|c|c|c|}
\hline & Market 1 & Market 2 & $\tau_{m n}$ & Intern. SE & Extern. SE & Observ. & Distance & /t-km \\
\hline \multirow[t]{24}{*}{ Tanzania } & Arusha & Dodoma & 0.0712 & $(0.0115)$ & $(0.0083)$ & 13 & 423 & 0.1682 \\
\hline & Arusha & Musoma & 0.0693 & $(0.0122)$ & $(0.0021)$ & 27 & 504 & 0.1375 \\
\hline & Arusha & Singida & 0.0642 & $(0.0104)$ & $(0.0159)$ & 37 & 350 & 0.1834 \\
\hline & Arusha & Tanga & 0.0464 & $(0.0074)$ & $(0.0019)$ & 37 & 436 & 0.1065 \\
\hline & Bukoba & Mwanza & 0.0628 & $(0.0086)$ & $(0.0017)$ & 63 & 434 & 0.1446 \\
\hline & Bukoba & Kampala & 0.1363 & $(0.0114)$ & $(0.0144)$ & 2 & 299 & 0.4560 \\
\hline & Bukoba & Mbarara & 0.1799 & $(0.0115)$ & $(0.0251)$ & 25 & 313 & 0.5747 \\
\hline & DaresSalaam & Dodoma & 0.0669 & $(0.0128)$ & $(0.0051)$ & 47 & 449 & 0.1490 \\
\hline & DaresSalaam & Iringa & 0.0686 & $(0.0038)$ & $(0.0077)$ & 174 & 501 & 0.1369 \\
\hline & DaresSalaam & Mtwara & 0.0640 & $(0.0068)$ & $(0.0047)$ & 52 & 559 & 0.1144 \\
\hline & DaresSalaam & Tanga & 0.0595 & $(0.0122)$ & $(0.0023)$ & 57 & 355 & 0.1675 \\
\hline & Dodoma & Iringa & 0.0765 & $(0.0033)$ & $(0.0046)$ & 174 & 265 & 0.2886 \\
\hline & Dodoma & Singida & 0.0925 & $(0.0112)$ & $(0.0118)$ & 37 & 250 & 0.3699 \\
\hline & Iringa & Mbeya & 0.0740 & $(0.0069)$ & $(0.0020)$ & 74 & 336 & 0.2201 \\
\hline & Iringa & Songea & 0.0594 & $(0.0030)$ & $(0.0098)$ & 134 & 436 & 0.1362 \\
\hline & Kigoma & Sumbawanga & 0.1178 & $(0.0113)$ & $(0.0046)$ & 34 & 536 & 0.2197 \\
\hline & Kigoma & Tabora & 0.1190 & $(0.0099)$ & $(0.0083)$ & 59 & 716 & 0.1663 \\
\hline & Mbeya & Songea & 0.0622 & $(0.0031)$ & $(0.0077)$ & 132 & 423 & 0.1472 \\
\hline & Mbeya & Sumbawanga & 0.0757 & $(0.0041)$ & $(0.0106)$ & 149 & 317 & 0.2388 \\
\hline & Mtwara & Songea & 0.0996 & $(0.0129)$ & $(0.0070)$ & 20 & 655 & 0.1521 \\
\hline & Musoma & Mwanza & 0.0743 & $(0.0089)$ & $(0.0056)$ & 67 & 223 & 0.3330 \\
\hline & Mwanza & Singida & 0.1024 & $(0.0104)$ & $(0.0085)$ & 38 & 475 & 0.2156 \\
\hline & Mwanza & Tabora & 0.0707 & $(0.0111)$ & $(0.0048)$ & 24 & 367 & 0.1927 \\
\hline & Singida & Tabora & 0.1839 & $(0.0175)$ & $(0.0078)$ & 16 & 357 & 0.5151 \\
\hline \multirow[t]{11}{*}{ Uganda } & Arua & Gulu & 0.0590 & $(0.0091)$ & $(0.0128)$ & 9 & 232 & 0.2545 \\
\hline & Arua & Juba & 0.5288 & $(0.0422)$ & $(0.0415)$ & 12 & 336 & 1.5737 \\
\hline & Gulu & Kampala & 0.0755 & $(0.0064)$ & $(0.0235)$ & 50 & 339 & 0.2227 \\
\hline & Gulu & Lira & 0.0580 & $(0.0036)$ & $(0.0072)$ & 50 & 135 & 0.4293 \\
\hline & Gulu & Masindi & 0.0877 & $(0.0059)$ & $(0.0127)$ & 14 & 175 & 0.5010 \\
\hline & Gulu & Juba & 0.8681 & $(0.0850)$ & $(0.0484)$ & 15 & 281 & 3.0892 \\
\hline & Jinja & Kampala & 0.0649 & $(0.0045)$ & $(0.0129)$ & 50 & 79 & 0.8214 \\
\hline & Kampala & Lira & 0.0864 & $(0.0073)$ & $(0.0017)$ & 34 & 342 & 0.2527 \\
\hline & Kampala & Masindi & 0.1442 & $(0.0459)$ & $(0.0178)$ & 3 & 214 & 0.6737 \\
\hline & Kampala & Mbarara & 0.3276 & $(0.0673)$ & $(0.0418)$ & 3 & 265 & 1.2364 \\
\hline & Lira & Masindi & 0.0702 & $(0.0090)$ & $(0.0162)$ & 14 & 180 & 0.3898 \\
\hline \multirow[t]{3}{*}{ Djibouti } & Djibouti & AddisAbaba & 0.1481 & $(0.0192)$ & $(0.0026)$ & 53 & 920 & 0.1610 \\
\hline & Djibouti & Dessie & 0.1333 & $(0.0151)$ & $(0.0066)$ & 98 & 548 & 0.2432 \\
\hline & Djibouti & DireDawa & 0.1411 & $(0.0197)$ & $(0.0080)$ & 58 & 323 & 0.4368 \\
\hline \multirow[t]{4}{*}{ Eritrea } & Asmara & Massawa & 0.4333 & $(0.0709)$ & $(0.0000)$ & 2 & 110 & 3.9394 \\
\hline & Asmara & Gondar & 0.6112 & $(0.0419)$ & $(0.2675)$ & 16 & 538 & 1.1360 \\
\hline & Asmara & Mekele & 0.5602 & $(0.0468)$ & $(0.2156)$ & 14 & 311 & 1.8012 \\
\hline & Asmara & Kassala & 0.6892 & $(0.0533)$ & $(0.2654)$ & 16 & 428 & 1.6103 \\
\hline
\end{tabular}


Table A11: Estimated Overland Trade Costs (6/10)

\begin{tabular}{|c|c|c|c|c|c|c|c|c|}
\hline & Market 1 & Market 2 & $\tau_{m n}$ & Intern. SE & Extern. SE & Observ. & Distance & /t-km \\
\hline \multirow[t]{23}{*}{ Ethiopia } & AddisAbaba & Awasa & 0.0843 & $(0.0046)$ & $(0.0049)$ & 222 & 272 & 0.3100 \\
\hline & AddisAbaba & BahirDar & 0.1069 & $(0.0112)$ & $(0.0089)$ & 44 & 551 & 0.1941 \\
\hline & AddisAbaba & Dessie & 0.0612 & $(0.0059)$ & $(0.0051)$ & 59 & 385 & 0.1590 \\
\hline & AddisAbaba & DireDawa & 0.0349 & $(0.0013)$ & $(0.0011)$ & 299 & 507 & 0.0688 \\
\hline & AddisAbaba & Jimma & 0.0490 & $(0.0018)$ & $(0.0041)$ & 283 & 306 & 0.1600 \\
\hline & AddisAbaba & Nekemte & 0.1202 & $(0.0064)$ & $(0.0382)$ & 147 & 325 & 0.3699 \\
\hline & Awasa & Gode & 0.1498 & $(0.0068)$ & $(0.0050)$ & 134 & 629 & 0.2382 \\
\hline & Awasa & Jimma & 0.1876 & $(0.0432)$ & $(0.0326)$ & 12 & 416 & 0.4509 \\
\hline & Awasa & Yabelo & 0.1260 & $(0.0077)$ & $(0.0059)$ & 96 & 295 & 0.4271 \\
\hline & BahirDar & Dessie & 0.0537 & $(0.0024)$ & $(0.0032)$ & 273 & 474 & 0.1134 \\
\hline & BahirDar & Gondar & 0.0389 & $(0.0016)$ & $(0.0020)$ & 224 & 176 & 0.2208 \\
\hline & BahirDar & Nekemte & 0.0575 & $(0.0027)$ & $(0.0120)$ & 148 & 399 & 0.1442 \\
\hline & BahirDar & AdDamazin & 0.1034 & $(0.0058)$ & $(0.0323)$ & 112 & 466 & 0.2219 \\
\hline & Dessie & Gondar & 0.0582 & $(0.0067)$ & $(0.0023)$ & 78 & 528 & 0.1102 \\
\hline & Dessie & Mekele & 0.0603 & $(0.0053)$ & $(0.0038)$ & 103 & 388 & 0.1555 \\
\hline & DireDawa & Jijiga & 0.1178 & $(0.0097)$ & $(0.0099)$ & 78 & 155 & 0.7597 \\
\hline & Gambela & Jimma & 0.1162 & $(0.0050)$ & $(0.0194)$ & 136 & 420 & 0.2768 \\
\hline & Gambela & Nekemte & 0.1511 & $(0.0056)$ & $(0.0222)$ & 92 & 390 & 0.3875 \\
\hline & Gambela & Malakal & 0.2816 & $(0.0831)$ & $(0.1943)$ & 11 & 445 & 0.6328 \\
\hline & Gode & Jijiga & 0.0934 & $(0.0092)$ & $(0.0100)$ & 40 & 572 & 0.1632 \\
\hline & Gondar & Mekele & 0.0468 & $(0.0022)$ & $(0.0158)$ & 221 & 599 & 0.0781 \\
\hline & Gondar & AlQadarif & 0.2117 & $(0.0274)$ & $(0.0211)$ & 31 & 357 & 0.5929 \\
\hline & Jimma & Nekemte & 0.0875 & $(0.0055)$ & $(0.0485)$ & 155 & 244 & 0.3588 \\
\hline \multirow[t]{8}{*}{ South Sudan } & Bor & Malakal & 0.4707 & $(0.0433)$ & $(0.0358)$ & 63 & 472 & 0.9971 \\
\hline & Bor & Juba & 0.2114 & $(0.0215)$ & $(0.0091)$ & 37 & 200 & 1.0571 \\
\hline & Juba & Rumbek & 0.7834 & $(0.0966)$ & $(0.0227)$ & 34 & 678 & 1.1554 \\
\hline & Malakal & AdDamazin & 0.4076 & $(0.0322)$ & $(0.1391)$ & 80 & 484 & 0.8421 \\
\hline & Malakal & Kadugli & 0.3680 & $(0.0331)$ & $(0.0954)$ & 79 & 334 & 1.1018 \\
\hline & Malakal & Kosti & 0.3487 & $(0.0350)$ & $(0.2340)$ & 69 & 500 & 0.6975 \\
\hline & Rumbek & Wau & 0.8301 & $(0.0749)$ & $(0.0139)$ & 42 & 226 & 3.6732 \\
\hline & Wau & Kadugli & 0.4991 & $(0.0542)$ & $(0.0012)$ & 48 & 610 & 0.8182 \\
\hline \multirow[t]{16}{*}{ Sudan } & AdDamazin & AlQadarif & 0.0683 & $(0.0032)$ & $(0.0068)$ & 169 & 523 & 0.1307 \\
\hline & AdDamazin & Khartoum & 0.1124 & $(0.0081)$ & $(0.0050)$ & 89 & 533 & 0.2109 \\
\hline & AdDamazin & Kosti & 0.0774 & $(0.0139)$ & $(0.0054)$ & 12 & 352 & 0.2198 \\
\hline & AlFashir & ElGeneina & 0.1326 & $(0.0108)$ & $(0.0068)$ & 126 & 352 & 0.3768 \\
\hline & AlFashir & ElObeid & 0.1442 & $(0.0127)$ & $(0.0088)$ & 98 & 613 & 0.2352 \\
\hline & AlFashir & Nyala & 0.0755 & $(0.0089)$ & $(0.0025)$ & 72 & 194 & 0.3894 \\
\hline & AlQadarif & Kassala & 0.0779 & $(0.0056)$ & $(0.0037)$ & 101 & 271 & 0.2873 \\
\hline & AlQadarif & Khartoum & 0.0662 & $(0.0032)$ & $(0.0024)$ & 254 & 356 & 0.1859 \\
\hline & AlQadarif & Kosti & 0.0360 & $(0.0018)$ & $(0.0042)$ & 209 & 396 & 0.0910 \\
\hline & ElGeneina & Nyala & 0.1391 & $(0.0050)$ & $(0.0180)$ & 240 & 376 & 0.3700 \\
\hline & ElGeneina & Abeche & 0.0555 & $(0.0026)$ & $(0.0017)$ & 217 & 197 & 0.2816 \\
\hline & ElObeid & Kadugli & 0.0941 & $(0.0043)$ & $(0.0250)$ & 211 & 262 & 0.3592 \\
\hline & ElObeid & Kosti & 0.0354 & $(0.0020)$ & $(0.0006)$ & 222 & 311 & 0.1139 \\
\hline & Kassala & PortSudan & 0.0951 & $(0.0032)$ & $(0.0068)$ & 252 & 575 & 0.1654 \\
\hline & Khartoum & Kosti & 0.0622 & $(0.0016)$ & $(0.0013)$ & 292 & 317 & 0.1963 \\
\hline & Khartoum & PortSudan & 0.0611 & $(0.0037)$ & $(0.0019)$ & 214 & 821 & 0.0744 \\
\hline
\end{tabular}


Table A11: Estimated Overland Trade Costs (7/10)

\begin{tabular}{|c|c|c|c|c|c|c|c|c|}
\hline & Market 1 & Market 2 & $\tau_{m n}$ & Intern. SE & Extern. SE & Observ. & Distance & $/ \mathrm{t}-\mathrm{km}$ \\
\hline \multirow[t]{12}{*}{ Benin } & Cotonou & Parakou & 0.1162 & $(0.0129)$ & $(0.0058)$ & 37 & 425 & 0.2735 \\
\hline & Cotonou & Lagos & 0.1875 & $(0.0154)$ & $(0.0214)$ & 113 & 120 & 1.5627 \\
\hline & Cotonou & Lome & 0.0957 & $(0.0041)$ & $(0.0129)$ & 240 & 147 & 0.6513 \\
\hline & Malanville & Parakou & 0.1461 & $(0.0763)$ & $(0.0276)$ & 4 & 320 & 0.4566 \\
\hline & Malanville & Niamey & 0.0834 & $(0.0034)$ & $(0.0055)$ & 88 & 297 & 0.2810 \\
\hline & Malanville & Sokoto & 0.1050 & $(0.0192)$ & $(0.0026)$ & 16 & 334 & 0.3144 \\
\hline & Natitingou & Parakou & 0.1500 & $(0.0685)$ & $(0.0193)$ & 4 & 217 & 0.6914 \\
\hline & Natitingou & FadaNgourma & 0.1718 & $(0.0287)$ & $(0.0019)$ & 15 & 253 & 0.6790 \\
\hline & Natitingou & Kara & 0.1051 & $(0.0068)$ & $(0.0071)$ & 110 & 119 & 0.8833 \\
\hline & Parakou & Ibadan & 0.1142 & $(0.0210)$ & $(0.0066)$ & 14 & 301 & 0.3794 \\
\hline & Parakou & Ilorin & 0.1164 & $(0.0094)$ & $(0.0921)$ & 70 & 284 & 0.4099 \\
\hline & Parakou & Kara & 0.1281 & $(0.0369)$ & $(0.0237)$ & 10 & 200 & 0.6407 \\
\hline \multirow{11}{*}{$\begin{array}{l}\text { Burkina } \\
\text { Faso }\end{array}$} & BoboDiou. & Dedougou & 0.0222 & $(0.0016)$ & $(0.0030)$ & 82 & 179 & 0.1238 \\
\hline & BoboDiou. & Ouagadougou & 0.0228 & $(0.0014)$ & $(0.0018)$ & 82 & 356 & 0.0639 \\
\hline & BoboDiou. & Wa & 0.1386 & $(0.0056)$ & $(0.0224)$ & 82 & 313 & 0.4429 \\
\hline & BoboDiou. & Mopti & 0.0532 & $(0.0106)$ & $(0.0139)$ & 8 & 475 & 0.1120 \\
\hline & BoboDiou. & Segou & 0.0675 & $(0.0055)$ & $(0.0145)$ & 4 & 377 & 0.1791 \\
\hline & BoboDiou. & Sikasso & 0.0215 & $(0.0017)$ & $(0.0012)$ & 82 & 176 & 0.1223 \\
\hline & Dedougou & Ouagadougou & 0.0263 & $(0.0013)$ & $(0.0039)$ & 128 & 225 & 0.1170 \\
\hline & FadaNgourma & Ouagadougou & 0.0538 & $(0.0088)$ & $(0.0024)$ & 33 & 223 & 0.2414 \\
\hline & FadaNgourma & Niamey & 0.1517 & $(0.0026)$ & $(0.0036)$ & 104 & 292 & 0.5196 \\
\hline & Ouagadougou & Bolgatanga & 0.0955 & $(0.0170)$ & $(0.0187)$ & 20 & 212 & 0.4505 \\
\hline & Ouagadougou & Kara & 0.1317 & $(0.0203)$ & $(0.0203)$ & 18 & 531 & 0.2481 \\
\hline \multirow[t]{12}{*}{ Cameroon } & Bamenda & Douala & 0.0686 & $(0.0042)$ & $(0.0070)$ & 158 & 305 & 0.2248 \\
\hline & Bamenda & Yaounde & 0.1053 & $(0.0031)$ & $(0.0052)$ & 200 & 368 & 0.2862 \\
\hline & Bamenda & Calabar & 0.1774 & $(0.0268)$ & $(0.0209)$ & 20 & 331 & 0.5361 \\
\hline & Bamenda & Enugu & 0.2563 & $(0.0205)$ & $(0.0217)$ & 2 & 511 & 0.5016 \\
\hline & Douala & Yaounde & 0.1092 & $(0.0058)$ & $(0.0010)$ & 101 & 236 & 0.4627 \\
\hline & Douala & Calabar & 0.1254 & $(0.0063)$ & $(0.0086)$ & 103 & 453 & 0.2768 \\
\hline & Douala & Enugu & 0.1697 & $(0.0154)$ & $(0.0117)$ & 30 & 632 & 0.2685 \\
\hline & Garoua & Yaounde & 0.1058 & $(0.0044)$ & $(0.0145)$ & 200 & 939 & 0.1127 \\
\hline & Garoua & Ndjamena & 0.1142 & $(0.0136)$ & $(0.0079)$ & 39 & 495 & 0.2306 \\
\hline & Garoua & Maiduguri & 0.0848 & $(0.0137)$ & $(0.0046)$ & 20 & 425 & 0.1995 \\
\hline & Garoua & Yola & 0.1072 & $(0.0141)$ & $(0.0038)$ & 33 & 167 & 0.6421 \\
\hline & Yaounde & Libreville & 0.2128 & $(0.0296)$ & $(0.0091)$ & 2 & 933 & 0.2281 \\
\hline \multirow[t]{5}{*}{ Chad } & Abeche & Ndjamena & 0.0980 & $(0.0068)$ & $(0.0042)$ & 95 & 753 & 0.1301 \\
\hline & Moundou & Ndjamena & 0.0796 & $(0.0053)$ & $(0.0012)$ & 84 & 474 & 0.1680 \\
\hline & Moundou & Sarh & 0.0600 & $(0.0150)$ & $(0.0038)$ & 18 & 305 & 0.1969 \\
\hline & Ndjamena & Sarh & 0.0700 & $(0.0059)$ & $(0.0005)$ & 103 & 558 & 0.1255 \\
\hline & Ndjamena & Maiduguri & 0.0956 & $(0.0136)$ & $(0.0073)$ & 52 & 260 & 0.3678 \\
\hline
\end{tabular}


Table A11: Estimated Overland Trade Costs (8/10)

\begin{tabular}{|c|c|c|c|c|c|c|c|c|}
\hline & Market 1 & Market 2 & $\tau_{m n}$ & Intern. SE & Extern. SE & Observ. & Distance & /t-km \\
\hline Cote & Abengourou & Abidjan & 0.1216 & $(0.0107)$ & $(0.0120)$ & 45 & 205 & 0.5932 \\
\hline \multirow[t]{13}{*}{ d'Ivoire } & Abengourou & Bouake & 0.2114 & $(0.0225)$ & $(0.0537)$ & 45 & 346 & 0.6108 \\
\hline & Abengourou & Kumasi & 0.1612 & $(0.0146)$ & $(0.0218)$ & 45 & 266 & 0.6059 \\
\hline & Abidjan & Bouake & 0.1468 & $(0.0127)$ & $(0.0254)$ & 50 & 351 & 0.4182 \\
\hline & Abidjan & Daloa & 0.0968 & $(0.0128)$ & $(0.0456)$ & 51 & 385 & 0.2513 \\
\hline & Abidjan & Sek.Tak. & 0.1635 & $(0.0274)$ & $(0.0019)$ & 19 & 321 & 0.5092 \\
\hline & Bouake & Daloa & 0.1068 & $(0.0113)$ & $(0.0222)$ & 50 & 241 & 0.4432 \\
\hline & Bouake & Sikasso & 0.0607 & $(0.0044)$ & $(0.0113)$ & 62 & 492 & 0.1234 \\
\hline & Daloa & Man & 0.0979 & $(0.0137)$ & $(0.0147)$ & 52 & 188 & 0.5205 \\
\hline & Man & Odienne & 0.0799 & $(0.0046)$ & $(0.0050)$ & 104 & 268 & 0.2982 \\
\hline & Man & Nzerekore & 0.2739 & $(0.0497)$ & $(0.0120)$ & 4 & 205 & 1.3363 \\
\hline & Man & Gbarnga & 0.0946 & $(0.0092)$ & $(0.0017)$ & 41 & 270 & 0.3505 \\
\hline & Odienne & Kankan & 0.1197 & $(0.0107)$ & $(0.0278)$ & 39 & 301 & 0.3978 \\
\hline & Odienne & Bamako & 0.0914 & $(0.0058)$ & $(0.0112)$ & 52 & 406 & 0.2251 \\
\hline \multirow[t]{2}{*}{ Gambia } & Banjul & Kaolack & 0.1662 & $(0.0354)$ & $(0.0259)$ & 5 & 153 & 1.0864 \\
\hline & Banjul & Ziguinchor & 0.0875 & $(0.0038)$ & $(0.0040)$ & 154 & 115 & 0.7612 \\
\hline \multirow[t]{14}{*}{ Ghana } & Accra & Но & 0.1589 & $(0.0307)$ & $(0.0330)$ & 31 & 156 & 1.0188 \\
\hline & Accra & Kumasi & 0.1309 & $(0.0055)$ & $(0.0231)$ & 347 & 253 & 0.5175 \\
\hline & Accra & Sek.Tak. & 0.1692 & $(0.0116)$ & $(0.0564)$ & 118 & 248 & 0.6824 \\
\hline & Accra & Lome & 0.2492 & $(0.1001)$ & $(0.0543)$ & 5 & 192 & 1.2978 \\
\hline & Bolgatanga & Tamale & 0.1485 & $(0.0541)$ & $(0.0389)$ & 2 & 172 & 0.8635 \\
\hline & Bolgatanga & Wa & 0.0485 & $(0.0016)$ & $(0.0036)$ & 347 & 264 & 0.1839 \\
\hline & Ho & Tamale & 0.1826 & $(0.0089)$ & $(0.0446)$ & 114 & 469 & 0.3893 \\
\hline & Ho & Kara & 0.1503 & $(0.0263)$ & $(0.0286)$ & 4 & 462 & 0.3252 \\
\hline & Ho & Lome & 0.2800 & $(0.0286)$ & $(0.0247)$ & 28 & 129 & 2.1704 \\
\hline & Kumasi & Sek.Tak. & 0.1084 & $(0.0121)$ & $(0.0128)$ & 51 & 281 & 0.3858 \\
\hline & Kumasi & Tamale & 0.1256 & $(0.0101)$ & $(0.0148)$ & 79 & 380 & 0.3305 \\
\hline & Kumasi & $\mathrm{Wa}$ & 0.0860 & $(0.0045)$ & $(0.0020)$ & 222 & 446 & 0.1928 \\
\hline & Tamale & $\mathrm{Wa}$ & 0.1587 & $(0.0287)$ & $(0.0359)$ & 2 & 303 & 0.5237 \\
\hline & Tamale & Kara & 0.1221 & $(0.0058)$ & $(0.0319)$ & 260 & 258 & 0.4733 \\
\hline \multirow[t]{11}{*}{ Guinea } & Conakry & Kankan & 0.1724 & $(0.0270)$ & $(0.0196)$ & 8 & 651 & 0.2648 \\
\hline & Conakry & Labe & 0.2316 & $(0.0189)$ & $(0.0216)$ & 7 & 404 & 0.5733 \\
\hline & Conakry & Nzerekore & 0.1077 & $(0.0220)$ & $(0.0078)$ & 3 & 845 & 0.1275 \\
\hline & Conakry & Freetown & 0.3976 & $(0.0460)$ & $(0.0288)$ & 4 & 311 & 1.2786 \\
\hline & Kankan & Labe & 0.0882 & $(0.0068)$ & $(0.0067)$ & 50 & 518 & 0.1703 \\
\hline & Kankan & Nzerekore & 0.2030 & $(0.1054)$ & $(0.0483)$ & 2 & 450 & 0.4511 \\
\hline & Kankan & Bamako & 0.1596 & $(0.0100)$ & $(0.0243)$ & 53 & 372 & 0.4291 \\
\hline & Labe & Nzerekore & 0.1482 & $(0.0394)$ & $(0.0244)$ & 4 & 727 & 0.2039 \\
\hline & Labe & Bissau & 0.2681 & $(0.0168)$ & $(0.0985)$ & 51 & 539 & 0.4974 \\
\hline & Labe & Tambacounda & 0.1789 & $(0.0763)$ & $(0.0000)$ & 2 & 422 & 0.4240 \\
\hline & Nzerekore & Gbarnga & 0.3618 & $(0.0372)$ & $(0.0247)$ & 6 & 168 & 2.1533 \\
\hline G. Bissau & Bissau & Ziguinchor & 1.0714 & $(0.0736)$ & $(0.0065)$ & 46 & 145 & 7.3892 \\
\hline \multirow[t]{2}{*}{ Liberia } & Gbarnga & Monrovia & 0.0513 & $(0.0060)$ & $(0.0003)$ & 33 & 196 & 0.2619 \\
\hline & Monrovia & Bo & 0.2382 & $(0.0024)$ & $(0.0265)$ & 3 & 357 & 0.6671 \\
\hline
\end{tabular}


Table A11: Estimated Overland Trade Costs (9/10)

\begin{tabular}{lcccccccc}
\hline & Market 1 & Market 2 & $\tau_{m n}$ & Intern. SE & Extern. SE & Observ. & Distance & /t-km \\
\hline \multirow{4}{*}{ Mali } & Bamako & Kayes & 0.0644 & $(0.0023)$ & $(0.0043)$ & 264 & 613 & 0.1051 \\
& Bamako & Segou & 0.0383 & $(0.0015)$ & $(0.0023)$ & 192 & 236 & 0.1621 \\
& Bamako & Sikasso & 0.0416 & $(0.0061)$ & $(0.0023)$ & 28 & 364 & 0.1144 \\
& Bamako & AdelBagrou & 0.1103 & $(0.0070)$ & $(0.0123)$ & 82 & 431 & 0.2559 \\
& Bamako & Tintane & 0.3943 & $(0.0733)$ & $(0.2202)$ & 17 & 704 & 0.5601 \\
& Gao & Mopti & 0.0435 & $(0.0029)$ & $(0.0065)$ & 163 & 583 & 0.0747 \\
& Gao & Niamey & 0.1270 & $(0.0032)$ & $(0.0099)$ & 98 & 447 & 0.2842 \\
& Kayes & Tintane & 0.3863 & $(0.0759)$ & $(0.2074)$ & 17 & 563 & 0.6861 \\
& Kayes & Tambacounda & 0.0878 & $(0.0117)$ & $(0.0031)$ & 43 & 284 & 0.3092 \\
& Mopti & Segou & 0.0506 & $(0.0037)$ & $(0.0030)$ & 52 & 401 & 0.1263 \\
& Segou & Sikasso & 0.0385 & $(0.0044)$ & $(0.0043)$ & 33 & 291 & 0.1323 \\
\hline \multirow{2}{*}{ Mauritania } & AdelBagrou & Tintane & 0.2659 & $(0.0488)$ & $(0.1617)$ & 17 & 479 & 0.5552 \\
& Nouakchott & Tintane & 0.1165 & $(0.0277)$ & $(0.0228)$ & 4 & 747 & 0.1560 \\
Nouakchott & SaintLouis & 0.4857 & $(0.0641)$ & $(0.0360)$ & 7 & 303 & 1.6029 \\
\hline Agadez & Arlit & 0.0532 & $(0.0055)$ & 0.0000 & 72 & 241 & 0.2207 \\
& Agadez & Tahoua & 0.0546 & $(0.0034)$ & $(0.0068)$ & 70 & 406 & 0.1344 \\
& Agadez & Zinder & 0.0771 & $(0.0024)$ & $(0.0011)$ & 193 & 446 & 0.1728 \\
& Diffa & Zinder & 0.0703 & $(0.0079)$ & $(0.0068)$ & 14 & 475 & 0.1479 \\
Diffa & Maiduguri & 0.0764 & $(0.0048)$ & $(0.0173)$ & 32 & 220 & 0.3472 \\
& Maradi & Niamey & 0.1763 & $(0.0065)$ & $(0.0250)$ & 2 & 664 & 0.2654 \\
& Maradi & Tahoua & 0.1108 & $(0.0039)$ & $(0.0160)$ & 70 & 352 & 0.3148 \\
Maradi & Zinder & 0.0325 & $(0.0095)$ & $(0.0074)$ & 11 & 238 & 0.1367 \\
Maradi & Katsina & 0.0902 & $(0.0279)$ & $(0.0100)$ & 11 & 92 & 0.9803 \\
& Maradi & Sokoto & 0.1053 & $(0.0388)$ & $(0.0146)$ & 6 & 342 & 0.3080 \\
Niamey & Tahoua & 0.0356 & $(0.0032)$ & $(0.0044)$ & 70 & 553 & 0.0643 \\
Niamey & Sokoto & 0.0666 & $(0.0157)$ & $(0.0057)$ & 10 & 513 & 0.1298 \\
& Tahoua & Sokoto & 0.0783 & $(0.0051)$ & $(0.0094)$ & 36 & 230 & 0.3406 \\
Zinder & Kano & 0.0700 & $(0.0230)$ & $(0.0155)$ & 8 & 240 & 0.2917 \\
\hline
\end{tabular}


Table A11: Estimated Overland Trade Costs (10/10)

\begin{tabular}{|c|c|c|c|c|c|c|c|c|}
\hline & Market 1 & Market 2 & $\tau_{m n}$ & Intern. SE & Extern. SE & Observ. & Distance & $/ \mathrm{t}-\mathrm{km}$ \\
\hline \multirow[t]{37}{*}{ Nigeria } & Abuja & Ilorin & 0.1351 & $(0.0727)$ & $(0.0306)$ & 2 & 453 & 0.2982 \\
\hline & Abuja & Jos & 0.0656 & $(0.0024)$ & $(0.0090)$ & 288 & 275 & 0.2387 \\
\hline & Abuja & Kaduna & 0.1263 & $(0.0259)$ & $(0.0261)$ & 8 & 211 & 0.5987 \\
\hline & Abuja & Lokoja & 0.0963 & $(0.0062)$ & $(0.0015)$ & 144 & 202 & 0.4767 \\
\hline & Abuja & Makurdi & 0.1837 & $(0.0716)$ & $(0.0349)$ & 4 & 283 & 0.6491 \\
\hline & Akure & BeninCity & 0.1252 & $(0.0049)$ & $(0.0299)$ & 283 & 172 & 0.7282 \\
\hline & Akure & Ibadan & 0.0957 & $(0.0030)$ & $(0.0124)$ & 282 & 178 & 0.5378 \\
\hline & Akure & Ilorin & 0.1327 & $(0.0060)$ & $(0.0036)$ & 197 & 203 & 0.6539 \\
\hline & BeninCity & Enugu & 0.1244 & $(0.0091)$ & $(0.0221)$ & 100 & 254 & 0.4899 \\
\hline & BeninCity & Ibadan & 0.1798 & $(0.0054)$ & $(0.0229)$ & 278 & 282 & 0.6375 \\
\hline & BeninCity & Lagos & 0.1629 & $(0.0660)$ & $(0.0347)$ & 13 & 326 & 0.4996 \\
\hline & BeninCity & Lokoja & 0.1561 & $(0.0119)$ & $(0.0069)$ & 90 & 276 & 0.5657 \\
\hline & BeninCity & PortHarcourt & 0.1326 & $(0.0061)$ & $(0.0225)$ & 287 & 332 & 0.3993 \\
\hline & Calabar & Enugu & 0.1540 & $(0.0269)$ & $(0.0072)$ & 129 & 263 & 0.5857 \\
\hline & Calabar & PortHarcourt & 0.2567 & $(0.0341)$ & $(0.0165)$ & 62 & 216 & 1.1886 \\
\hline & Enugu & Makurdi & 0.1455 & $(0.0153)$ & $(0.0117)$ & 63 & 257 & 0.5662 \\
\hline & Enugu & PortHarcourt & 0.1679 & $(0.0126)$ & $(0.0226)$ & 172 & 233 & 0.7204 \\
\hline & Enugu & Yola & 0.1830 & $(0.0111)$ & $(0.0054)$ & 133 & 761 & 0.2404 \\
\hline & Gombe & Jos & 0.0859 & $(0.0030)$ & $(0.0142)$ & 272 & 279 & 0.3080 \\
\hline & Gombe & Maiduguri & 0.1146 & $(0.0457)$ & $(0.0329)$ & 3 & 319 & 0.3594 \\
\hline & Gombe & Yola & 0.0862 & $(0.0092)$ & $(0.0096)$ & 96 & 250 & 0.3447 \\
\hline & Ibadan & Ilorin & 0.0703 & $(0.0046)$ & $(0.0019)$ & 177 & 159 & 0.4420 \\
\hline & Ibadan & Lagos & 0.2131 & $(0.0164)$ & $(0.0181)$ & 121 & 142 & 1.5005 \\
\hline & Ilorin & Kaduna & 0.0573 & $(0.0056)$ & $(0.0030)$ & 127 & 492 & 0.1164 \\
\hline & Ilorin & Lokoja & 0.2157 & $(0.0444)$ & $(0.0373)$ & 6 & 326 & 0.6617 \\
\hline & Ilorin & Sokoto & 0.0678 & $(0.0024)$ & $(0.0049)$ & 276 & 682 & 0.0994 \\
\hline & Jos & Kaduna & 0.0605 & $(0.0027)$ & $(0.0077)$ & 280 & 274 & 0.2208 \\
\hline & Jos & Kano & 0.0791 & $(0.0030)$ & $(0.0078)$ & 284 & 392 & 0.2018 \\
\hline & Jos & Maiduguri & 0.1016 & $(0.0206)$ & $(0.0271)$ & 4 & 587 & 0.1730 \\
\hline & Jos & Makurdi & 0.0874 & $(0.0101)$ & $(0.0121)$ & 77 & 310 & 0.2818 \\
\hline & Kaduna & Kano & 0.0529 & $(0.0026)$ & $(0.0046)$ & 288 & 233 & 0.2271 \\
\hline & Kaduna & Sokoto & 0.0588 & $(0.0055)$ & $(0.0010)$ & 84 & 468 & 0.1255 \\
\hline & Kano & Katsina & 0.0614 & $(0.0061)$ & $(0.0013)$ & 91 & 173 & 0.3547 \\
\hline & Kano & Maiduguri & 0.0748 & $(0.0099)$ & $(0.0088)$ & 29 & 636 & 0.1176 \\
\hline & Kano & Sokoto & 0.0912 & $(0.0297)$ & $(0.0108)$ & 7 & 540 & 0.1689 \\
\hline & Maiduguri & Yola & 0.1032 & $(0.0167)$ & $(0.0301)$ & 62 & 406 & 0.2542 \\
\hline & Makurdi & Yola & 0.0811 & $(0.0032)$ & $(0.0311)$ & 276 & 617 & 0.1314 \\
\hline \multirow[t]{7}{*}{ Senegal } & Dakar & Kaolack & 0.0778 & $(0.0045)$ & $(0.0016)$ & 80 & 214 & 0.3636 \\
\hline & Dakar & SaintLouis & 0.0457 & $(0.0022)$ & $(0.0017)$ & 152 & 245 & 0.1864 \\
\hline & Dakar & Touba & 0.0752 & $(0.0027)$ & $(0.0147)$ & 135 & 181 & 0.4155 \\
\hline & Kaolack & Tambacounda & 0.0404 & $(0.0033)$ & $(0.0016)$ & 73 & 375 & 0.1077 \\
\hline & Kaolack & Touba & 0.0366 & $(0.0021)$ & $(0.0030)$ & 177 & 154 & 0.2379 \\
\hline & SaintLouis & Touba & 0.0940 & $(0.0114)$ & $(0.0092)$ & 7 & 176 & 0.5341 \\
\hline & Tambacounda & Ziguinchor & 0.1128 & $(0.0267)$ & $(0.0152)$ & 6 & 408 & 0.2765 \\
\hline \multirow[t]{3}{*}{ S. Leone } & Bo & Freetown & 0.0622 & $(0.0076)$ & $(0.0037)$ & 25 & 237 & 0.2624 \\
\hline & Bo & Kabala & 0.0679 & $(0.0153)$ & $(0.0273)$ & 18 & 253 & 0.2684 \\
\hline & Freetown & Kabala & 0.0667 & $(0.0253)$ & $(0.0102)$ & 3 & 302 & 0.2209 \\
\hline Togo & Kara & Lome & 0.1858 & $(0.0092)$ & $(0.0118)$ & 97 & 412 & 0.4510 \\
\hline
\end{tabular}


Table A12: Estimated Port-to-World-Market Trade Costs

\begin{tabular}{|c|c|c|c|c|c|c|}
\hline & Market 1 & Market 2 & $\tau_{m n}$ & Internal SE & External SE & Observ. \\
\hline \multirow[t]{6}{*}{ Mozambique } & Beira & Gulf & 0.1084 & $(0.0166)$ & $(0.0036)$ & 36 \\
\hline & Beira & Bangkok & 0.2419 & $(0.0160)$ & $(0.0057)$ & 61 \\
\hline & Maputo & Gulf & 0.1838 & $(0.0103)$ & $(0.0146)$ & 65 \\
\hline & Maputo & Bangkok & 0.2092 & $(0.0083)$ & $(0.0000)$ & 120 \\
\hline & Nacala & Gulf & 0.0732 & $(0.0096)$ & $(0.0099)$ & 28 \\
\hline & Nacala & Bangkok & 0.2384 & $(0.0144)$ & $(0.0078)$ & 26 \\
\hline Namibia & Swakopmund & Gulf & 0.2751 & $(0.0170)$ & $(0.0128)$ & 4 \\
\hline Angola & Luanda & Gulf & 1.4519 & $(0.0116)$ & $(0.0000)$ & 4 \\
\hline Congo & PointeNoire & Bangkok & 0.6152 & $(0.0649)$ & $(0.0000)$ & 10 \\
\hline \multirow[t]{2}{*}{ D.R. Congo } & Matadi & Gulf & 0.3994 & $(0.0239)$ & $(0.0019)$ & 55 \\
\hline & Matadi & Bangkok & 0.7986 & $(0.0492)$ & $(0.0228)$ & 57 \\
\hline Kenya & Mombasa & Gulf & 0.1000 & $(0.0070)$ & $(0.0030)$ & 64 \\
\hline \multirow{3}{*}{ Somalia } & Bosaso & Gulf & 0.3319 & $(0.0142)$ & $(0.0015)$ & 184 \\
\hline & Kismayo & Gulf & 0.1182 & $(0.0112)$ & $(0.0012)$ & 55 \\
\hline & Mogadishu & Gulf & 0.0764 & $(0.0067)$ & $(0.0062)$ & 117 \\
\hline \multirow[t]{2}{*}{ Tanzania } & DaresSalaam & Gulf & 0.1062 & $(0.0117)$ & $(0.0032)$ & 33 \\
\hline & DaresSalaam & Bangkok & 0.3191 & $(0.0137)$ & $(0.0181)$ & 87 \\
\hline \multirow[t]{2}{*}{ Djibouti } & Djibouti & Gulf & 0.0912 & $(0.0032)$ & 0.0000 & 112 \\
\hline & Djibouti & Bangkok & 0.2737 & $(0.0095)$ & $(0.0000)$ & 112 \\
\hline Eritrea & Massawa & Gulf & 1.5038 & $(0.0754)$ & $(0.3223)$ & 9 \\
\hline Sudan & PortSudan & Gulf & 0.2723 & $(0.0113)$ & $(0.0017)$ & 137 \\
\hline \multirow[t]{2}{*}{ Benin } & Cotonou & Gulf & 0.2571 & $(0.0182)$ & $(0.0211)$ & 53 \\
\hline & Cotonou & Bangkok & 0.4291 & $(0.0071)$ & $(0.0016)$ & 120 \\
\hline \multirow[t]{2}{*}{ Cameroon } & Douala & Gulf & 0.3615 & $(0.0072)$ & $(0.0091)$ & 100 \\
\hline & Douala & Bangkok & 0.2482 & $(0.0067)$ & $(0.0014)$ & 97 \\
\hline \multirow[t]{2}{*}{ Cote d'Ivoire } & Abidjan & Gulf & 0.2730 & $(0.0127)$ & $(0.0053)$ & 26 \\
\hline & Abidjan & Bangkok & 0.2413 & $(0.0178)$ & $(0.0034)$ & 25 \\
\hline Gabon & Libreville & Bangkok & 0.5394 & $(0.0119)$ & $(0.0006)$ & 74 \\
\hline \multirow[t]{2}{*}{ Gambia } & Banjul & Gulf & 0.3193 & $(0.0046)$ & $(0.0154)$ & 156 \\
\hline & Banjul & Bangkok & 0.1151 & $(0.0093)$ & $(0.0002)$ & 61 \\
\hline \multirow[t]{2}{*}{ Ghana } & Accra & Gulf & 0.2816 & $(0.0082)$ & $(0.0188)$ & 112 \\
\hline & Accra & Bangkok & 0.4399 & $(0.0315)$ & $(0.0830)$ & 73 \\
\hline Guinea & Conakry & Bangkok & 0.5813 & $(0.0478)$ & $(0.0144)$ & 9 \\
\hline \multirow{2}{*}{ Guinea Bissau } & Bissau & Gulf & 1.5468 & $(0.1344)$ & $(0.0483)$ & 8 \\
\hline & Bissau & Bangkok & 0.4296 & $(0.0304)$ & $(0.0336)$ & 32 \\
\hline Liberia & Monrovia & Bangkok & 0.1156 & $(0.0117)$ & $(0.0007)$ & 39 \\
\hline \multirow[t]{2}{*}{ Mauritania } & Nouakchott & Gulf & 0.6870 & $(0.0536)$ & $(0.0455)$ & 11 \\
\hline & Nouakchott & Bangkok & 0.1219 & $(0.0048)$ & $(0.0024)$ & 48 \\
\hline \multirow[t]{4}{*}{ Nigeria } & Lagos & Gulf & 0.4879 & $(0.0309)$ & $(0.0469)$ & 47 \\
\hline & Lagos & Bangkok & 0.5720 & $(0.0337)$ & $(0.0055)$ & 41 \\
\hline & PortHarcourt & Gulf & 0.4651 & $(0.0096)$ & $(0.0752)$ & 147 \\
\hline & PortHarcourt & Bangkok & 0.6436 & $(0.0201)$ & $(0.0161)$ & 74 \\
\hline \multirow[t]{2}{*}{ Senegal } & Dakar & Gulf & 0.2202 & $(0.0062)$ & $(0.0055)$ & 88 \\
\hline & Dakar & Bangkok & 0.0919 & $(0.0280)$ & $(0.0020)$ & 8 \\
\hline Sierra Leone & Freetown & Bangkok & 0.2129 & $(0.0257)$ & $(0.0000)$ & 10 \\
\hline \multirow[t]{2}{*}{ Togo } & Lome & Gulf & 0.3925 & $(0.0366)$ & $(0.0317)$ & 14 \\
\hline & Lome & Bangkok & 0.3816 & $(0.0136)$ & $(0.0030)$ & 120 \\
\hline
\end{tabular}


Table A13: Correlation of Port-to-World-Market Trade Costs with Port Characteristics

\begin{tabular}{lcc}
\hline & $(1)$ & $(2)$ \\
\hline Population < 500,000 & 0.184 & 0.169 \\
& $(0.151)$ & $(0.142)$ \\
High volume & -0.0213 & -0.0378 \\
& $(0.0990)$ & $(0.124)$ \\
Corruption index & -0.0109 & -0.0208 \\
& $(0.0102)$ & $(0.0121)$ \\
LPI customs index & 0.184 & 0.241 \\
& $(0.237)$ & $(0.209)$ \\
GDP per capita & $5.07 \mathrm{E}-05$ & $4.89 \mathrm{E}-05$ \\
& $(3.21 \mathrm{E}-05)$ & $(3.02 \mathrm{E}-05)$ \\
Import tariff & & $4.06 \mathrm{E}-04$ \\
& & $(0.00176)$ \\
Constant & 0.203 & 0.404 \\
& $(0.430)$ & $(0.354)$ \\
Observations & 47 & 43 \\
Clusters & 25 & 23 \\
\hline
\end{tabular}

Note: Robust standard errors in () clustered by country. Tariff data was unavailable for Liberia and Somalia, so they and their 4 ports are excluded from column (2). 


\section{A8 Estimation: Price Differences Elsewhere}

In this section, I assess whether my counterfactual trade costs of $\$ 0.05 / \mathrm{t}-\mathrm{km}$ for overland market links and \$50/tonne for port-to-world-market links are in line with observed price differences within the US and between major global maize ports. Importantly, I do not develop a full model of production, consumption, storage, and trade as I do for Africa, so I do not identify when and where trade occurs. The price differences I consider are thus lower bounds on the actual trade costs, with the upper end of the range of price differences likely closest to actual trade costs.

I start by considering price series of maize for the 8 major US markets included in the USDA Feed Grains Database. I identify 11 transportation links along which direct trade between pairs of these markets is feasible. The median pair-wise price difference across all 1,320 observations during my period of interest (May 2003 - April 2013) is $\$ 0.012 / \mathrm{t}-\mathrm{km}$, with only 15 observations (1.1 percent) higher than $\$ 0.05 / \mathrm{t}-\mathrm{km}$. Details for each link are shown in table A14. Given that the US is a major maize exporter through the Gulf ports near New Orleans, this evidence is highly suggestive that trade costs within the US rarely if ever exceed $\$ 0.05 / \mathrm{t}-\mathrm{km}$.

Table A14: Domestic US Price Differences

\begin{tabular}{lccccc}
\hline Market 1 & Market 2 & Avg. Difference & Distance $(\mathrm{km})$ & Avg. /t-km & Percent $>\$ 0.05$ \\
\hline Chicago & Minneapolis & 0.0115 & 657 & 0.0175 & 0 \\
Chicago & Omaha & 0.0061 & 758 & 0.0081 & 0 \\
Chicago & St. Louis & 0.0053 & 478 & 0.0110 & 0.83 \\
Chicago & Toledo & 0.0036 & 394 & 0.0092 & 0 \\
Kansas City & Minneapolis & 0.0097 & 702 & 0.0139 & 0 \\
Kansas City & Omaha & 0.0035 & 303 & 0.0115 & 0 \\
Kansas City & St. Louis & 0.0077 & 399 & 0.0193 & 2.50 \\
Memphis & New Orleans & 0.0183 & 636 & 0.0287 & 6.67 \\
Memphis & St. Louis & 0.0039 & 455 & 0.0085 & 1.67 \\
Minneapolis & Omaha & 0.0075 & 615 & 0.0121 & 0 \\
Minneapolis & St. Louis & 0.0134 & 900 & 0.0148 & 0.83 \\
\hline
\end{tabular}

I next consider prices at the major maize export ports for the US, Argentina, and Ukraine, which are the first, second, and fifth largest exporters of maize globally (Brazil and China are third and fourth). Pairing each of these three markets together and computing monthly price differences for my study period as above I find an average price difference of $\$ 0.015$ ( $\$ 15 /$ tonne), with only 2.8 percent of observations higher than $\$ 0.05$ ( $\$ 50 /$ tonne). Details for each pair are shown in table A15. Although these ports may not regularly trade directly with each other, this evidence suggests that trade costs between major global maize ports do not significantly exceed $\$ 50 /$ tonne.

Table A15: Price Differences Between Major Global Maize Ports

\begin{tabular}{lccc}
\hline Market 1 & Market 2 & Avg. Difference & Percent $>\$ 0.05$ \\
\hline US Gulf & Argentina & 0.0106 & 0 \\
US Gulf & Ukraine & 0.0185 & 3.96 \\
Argentina & Ukraine & 0.0170 & 4.95 \\
\hline
\end{tabular}




\section{A9 Results: Comparison to Static Annual Model}

To implement a static annual model, I remove storage $S_{i m t}$ and associated costs $r_{m}$ and $k_{m}$ from the model. There are now 10 time periods (years) instead of 120 (months). In each time period, traders must decide how much of the local harvest $H_{i m t}$ to trade with other markets $\left(T_{\text {imnt }}\right)$ and to sell for local consumption $\left(Q_{i m t}\right)$. The market clearing condition from the main paper becomes:

$$
Q_{i m t}=H_{i m t}-\sum_{n \neq m} T_{i m n t}
$$

While the spatial arbitrage conditions still hold, there are no temporal arbitrage conditions, so equilibrium for each year can be solved independently. I proceed to re-estimate trade costs

$\tau_{m n}$ using the same iterative algorithm as before with the price series from my baseline estimated model as my price data. Trade cost estimates converge in 6 iterations.

Trade cost estimates are 23.4 percent lower on average using the static annual model. The static annual model underestimates 12.2 percent of the trade cost parameters by $50-97$ percent, 25.2 percent by 25-50 percent, 33.0 percent by 10-25 percent, and 19.1 percent by $2-10$ percent. The percentage change for the remaining 10.4 percent of parameters is between -2 and +2 percent.

I next re-solve the model under counterfactual trade costs and compare equilibrium outcomes to those under the (underestimated) baseline trade costs. It is not surprising that the effects of reducing trade costs are smaller. The overall welfare gain is 33.6 percent smaller than under the dynamic monthly model with storage. Of the 229 markets excluding Johannesburg, the welfare effects for 23.6 percent are underestimated by 50-98 percent, 30.1 percent by $25-50$ percent, 23.6 percent by 10-25 percent, and 7.4 percent by $0.5-10$ percent, while 7.4 percent of markets have a welfare effect that changes sign and 7.9 percent of markets have a welfare effect that is overestimated.

The intuition for the underestimation of trade costs and welfare effects is clear from the example in figure A1. For ease of illustration, I consider a case in which trade occurs between an African port and the world market. The left panel shows baseline maize price series in Accra, Ghana and the US Gulf, as well as the parity price for imports from the US Gulf to Accra under baseline trade costs. As is clear from the figure, Accra is an importer of maize. In keeping with Proposition 2, traders in Accra store maize first and import maize later, so that maize prices are significantly below import parity at harvest time and then increase to reach import parity as local stocks are consumed. Whereas my dynamic model estimates trade costs using price differences during the months when trade occurs at the end of the harvest cycle (i.e. from the peaks in the blue line to the black line), the static annual model using farm-gate prices estimates trade costs at the beginning of the harvest cycle (i.e. from the troughs in the blue line to the black line). The static annual model underestimates trade costs between the US Gulf and Accra by 22 percent. The right panel shows counterfactual maize price series in Accra (in red) as well as the parity price for imports from the US Gulf to Accra under counterfactual trade costs. The welfare effect of lowering trade costs depends on the change in prices, which for the dynamic monthly model is the difference between the blue and the red lines. In contrast, in the static annual model the change in prices is the difference between the troughs in the blue line and the dashed black line. This change in prices is always less than in the dynamic monthly model. The static annual model underestimates the welfare effect of lowering trade costs for Accra by 24 percent.

For inland market pairs, the changes that take place under the counterfactual are less easily visualized as they depend on interactions between multiple markets. However, figure A2 illustrates the difference between the two models using baseline maize price series in Butembo, D.R. Congo 

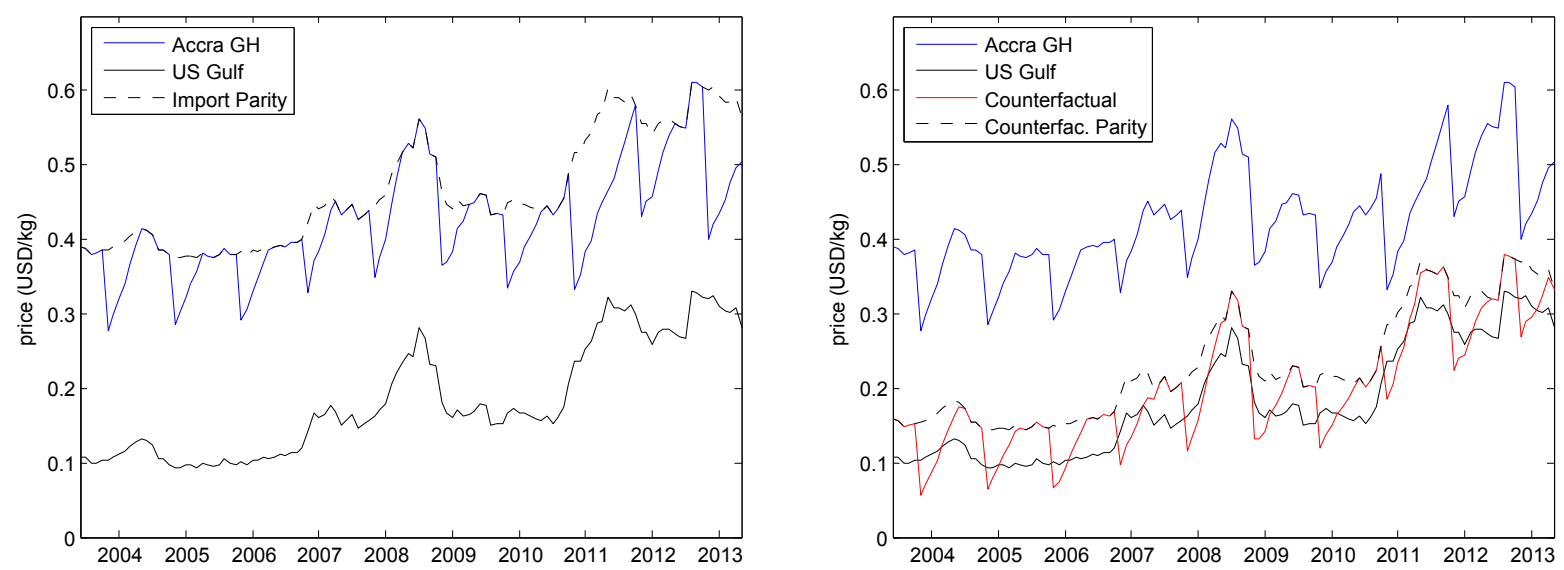

Figure A1: Maize Price Series for Accra, Ghana and the US Gulf Under Baseline and Counterfactual Trade Costs, May 2003 - April 2013

and Mbarara, Uganda, as well as the parity price for imports from Mbarara to Butembo under baseline trade costs. These markets are both in the equatorial zone with two annual harvests. As in the previous case, traders in Butembo store maize first and import maize later, so that maize prices are significantly below import parity at harvest time and then increase to reach import parity as local stocks are consumed. Again, the dynamic model estimates trade costs using price differences during the months when trade occurs at the end of the harvest cycle (i.e. from the peaks in the blue line to the black line), while the static annual model using farm-gate prices estimates trade costs at the beginning of the harvest cycle (i.e. from the troughs in the blue line to the black line). The static annual model underestimates trade costs between Mbarara and Butembo by 58 percent and the welfare effect of lowering trade costs for Butembo by 37 percent.

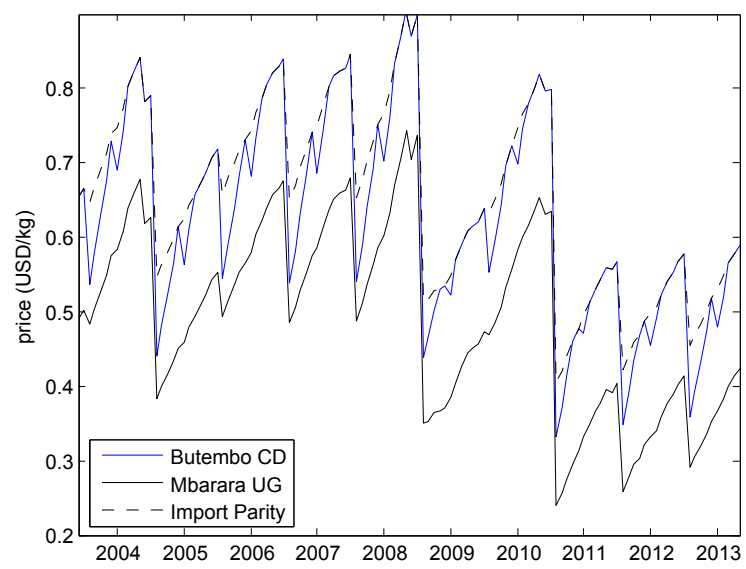

Figure A2: Maize Price Series for Butembo, D.R. Congo and Mbarara, Uganda Under Baseline Trade Costs, May 2003 - April 2013 


\section{A10 Results: Corridor Selection Exercise}

This section describes in greater detail the selection of overland links for the trade corridor extension in the main paper. I first compared the absolute difference in welfare between the "only sea" scenario in which only port-to-world-market costs are reduced and the main counterfactual in which all trade costs are reduced. Table A16 shows these differences for the 34 markets with a welfare difference of over $\$ 500$ million equivalent variation, which together account for 65.5 percent of the total welfare difference. 28 of these 34 markets are from only 4 countries (Nigeria, South Sudan, Sudan, and Ethiopia). I then identified 30 critical links for closing the welfare gap by looking at equilibrium trade flows to and from each of the 34 markets.

Of the 34 markets in Table A16, 6 (Enugu, Benin City, and Ibadan, Nigeria; Wau, Rumbek, and Juba, South Sudan) have a welfare difference of over $\$ 2$ billion equivalent variation each. I identified 6 critical links (in bold) for these markets and tried reducing trade costs along each of these links individually or in combination in order to identify the 1 and 5 links for which trade cost reduction would have the biggest effect (reported in the main paper).

Table A17 shows the 24 markets in 14 countries which have a welfare gap of over $\$ 300$ million equivalent variation after my initial reduction of trade costs along the 30 critical links. I proceeded to identify an additional 45 links important for closing the welfare gap for these markets. 88 percent of the welfare gains from reducing trade costs everywhere can be achieved by lowering trade costs on the 75 links in tables A16 and A17 along with port-to-world-market trade costs. A map of the 75 targeted links is included in figure A3.

My corridor selection exercise suggests that certain types of trade corridors may be particularly beneficial. First, reducing trade costs all the way from the world market to "dry ports" in denselypopulated inland areas can achieve significant welfare gains even if trade costs to areas further inland remain high. Table A18 shows the welfare effects of reducing trade costs along a single link between the port of Matadi in the Democratic Republic of Congo and the capital city Kinshasa. The table compares the potential gains from trade cost reduction on all links with the gains achieved by reducing trade costs on this single link, both for Kinshasa and for the seven further inland markets in western D.R. Congo that are either directly linked to Kinshasa or have only a single market in between. Reducing trade costs for this one link results in 53.7 percent of the welfare gain achievable by reducing trade costs on all 11 links connecting these markets. This is due both to the large population in the Kinshasa market catchment, which accounts for 39.5 percent of the potential welfare gains for the eight markets, as well as the secondary effects on the other seven markets, which achieve 26.9 percent of their potential welfare gains as lower prices in Kinshasa translate into lower prices everywhere despite continued high trade costs further inland.

Second, reducing trade costs along major inland corridors with significant imbalances or fluctuations in production and consumption can lead to major gains. My second, 75-link corridor counterfactual includes trade cost reduction along the complete east-west trans-Sahelian highway from Dakar, Senegal to Port Sudan, Sudan (22 links). This route traverses 7 countries (including 4 land-locked countries) which are major producers of millet and sorghum and include 6 of the 8 countries with the highest estimated per-capita grain demand parameters $\left(A_{m}\right)$. The Sahel is also subject to large fluctuations in local harvests due to its arid climate and erratic rainfall. Of a total possible welfare gain of $\$ 65.2$ billion equivalent variation from reducing trade costs on all 413 overland links, the 23 markets on the trans-Sahelian highway gain $\$ 9.1$ billion (14.0 percent) in my 75 -link corridor counterfactual. This figure increases to $\$ 15.2$ billion (23.3 percent) when the 35 markets with direct connections to one of these 23 markets are included.

Finally, targeting inland areas isolated by extreme trade costs can lead to very large gains. The 
five markets of South Sudan are perhaps the continent's most isolated grain deficit areas. Together they account for $\$ 10.5$ billion (16.1 percent) of the total possible welfare gain from reducing trade costs on all 413 overland links. My 75-link corridor counterfactual achieves all of these gains.

Table A19 lists the 90 links corresponding most closely to the proposed Trans-African Highways. The table is organized according to the 9 proposed highways (African Development Bank and UNECA 2003). The first 4 highways all begin in North Africa - the portions of these highways in and connecting to North Africa are excluded as they are not part of my network. Otherwise, I have kept as close to the proposed routes as possible. If a link is on more than one highway it is only listed once. Several of the highways are not complete in my network due to missing markets from my ideal list (in Angola, Cameroon, Central African Republic, and Guinea). 
Table A16: Initial Targeting of 30 Overland Links

\begin{tabular}{|c|c|c|c|}
\hline Country & Market & Welfare Diff. & 30 Targeted Links \\
\hline $\begin{array}{l}\text { Nigeria/ } \\
\text { Niger }\end{array}$ & $\begin{array}{c}\text { Enugu } \\
\text { BeninCity } \\
\text { Ibadan } \\
\text { PortHarcourt } \\
\text { Kano } \\
\text { Lagos } \\
\text { Akure } \\
\text { Calabar } \\
\text { Tahoua } \\
\text { Maiduguri } \\
\text { Makurdi } \\
\text { Ilorin } \\
\text { Lokoja } \\
\text { Gombe } \\
\text { Maradi }\end{array}$ & $\begin{array}{l}3187 \\
2969 \\
2542 \\
1776 \\
1547 \\
1386 \\
1294 \\
931 \\
668 \\
658 \\
593 \\
575 \\
569 \\
523 \\
520\end{array}$ & $\begin{array}{c}\text { PortHarcourt-Enugu } \\
\text { Lagos-BeninCity } \\
\text { Lagos-Ibadan } \\
\text { Enugu-Makurdi } \\
\text { Kano-Katsina } \\
\text { BeninCity-Enugu } \\
\text { Ibadan-Ilorin } \\
\text { PortHarcourt-Calabar } \\
\text { Katsina-Maradi } \\
\text { Kaduna-Kano } \\
\text { Makurdi-Jos } \\
\text { Ilorin-Kaduna } \\
\text { Jos-Kano }\end{array}$ \\
\hline $\begin{array}{l}\text { South Sudan/ } \\
\text { Sudan/ } \\
\text { Uganda }\end{array}$ & $\begin{array}{c}\text { Wau } \\
\text { Rumbek } \\
\text { Juba } \\
\text { Malakal } \\
\text { Bor } \\
\text { Nyala } \\
\text { AlFashir } \\
\text { ElGeneina } \\
\text { Kosti } \\
\text { Mbarara }\end{array}$ & $\begin{array}{c}2527 \\
2473 \\
2071 \\
1723 \\
1718 \\
1620 \\
1098 \\
869 \\
611 \\
573\end{array}$ & $\begin{array}{c}\text { Rumbek-Wau } \\
\text { Juba-Rumbek } \\
\text { Gulu-Juba } \\
\text { Kosti-Malakal } \\
\text { Juba-Bor } \\
\text { Kosti-ElObeid } \\
\text { ElObeid-AlFashir } \\
\text { AlFashir-ElGeneina } \\
\text { Khartoum-Kosti } \\
\text { Kampala-Mbarara } \\
\text { Kampala-Gulu }\end{array}$ \\
\hline Ethiopia & $\begin{array}{c}\text { Awasa } \\
\text { Dessie } \\
\text { BahirDar } \\
\text { AddisAbaba } \\
\text { Yabelo } \\
\text { Mekele }\end{array}$ & $\begin{array}{l}1176 \\
1003 \\
977 \\
852 \\
711 \\
658 \\
\end{array}$ & $\begin{array}{c}\text { AddisAbaba-Awasa } \\
\text { Djibouti-Dessie } \\
\text { AddisAbaba-BahirDar } \\
\text { Djibouti-AddisAbaba }\end{array}$ \\
\hline Mali & Bamako & 912 & \\
\hline D.R. Congo & Kinshasa & 876 & Matadi-Kinshasa \\
\hline Ghana & Kumasi & 560 & Accra-Kumasi \\
\hline
\end{tabular}


Table A17: Targeting of 45 Additional Overland Links

\begin{tabular}{|c|c|c|c|}
\hline Country & Market & Welfare Diff. & 45 Additional Targeted Links \\
\hline $\begin{array}{l}\text { Nigeria/ } \\
\text { Niger/ } \\
\text { Chad }\end{array}$ & $\begin{array}{l}\text { Maiduguri } \\
\text { Akure } \\
\text { Zinder } \\
\text { Lokoja } \\
\text { Gombe } \\
\text { Ndjamena } \\
\text { Tahoua }\end{array}$ & $\begin{array}{l}915 \\
610 \\
586 \\
491 \\
468 \\
451 \\
405\end{array}$ & $\begin{array}{l}\text { Kano-Maiduguri } \\
\text { Ibadan-Akure } \\
\text { Maradi-Zinder } \\
\text { BeninCity-Lokoja } \\
\text { Jos-Gombe } \\
\text { Maiduguri-Ndjamena } \\
\text { Maradi-Tahoua } \\
\text { Maradi-Niamey } \\
\text { Ndjamena-Abeche }\end{array}$ \\
\hline $\begin{array}{l}\text { South Sudan/ } \\
\text { Sudan/ } \\
\text { Uganda }\end{array}$ & $\begin{array}{c}\text { Wau } \\
\text { Juba } \\
\text { Bor } \\
\text { Kampala } \\
\text { Rumbek } \\
\text { Nyala } \\
\text { Mbarara }\end{array}$ & $\begin{array}{c}1578 \\
966 \\
910 \\
875 \\
767 \\
679 \\
404\end{array}$ & $\begin{array}{c}\text { Kadugli-Wau } \\
\text { ElObeid-Kadugli } \\
\text { Malakal-Bor } \\
\text { Jinja-Kampala } \\
\text { Abeche-ElGeneina } \\
\text { AlFashir-Nyala } \\
\text { Kigali-Mbarara } \\
\text { Mombasa-Nairobi } \\
\text { Nairobi-Nakuru } \\
\text { Nakuru-Eldoret } \\
\text { Eldoret-Jinja } \\
\text { PortSudan-Kassala } \\
\text { Kassala-AlQadarif } \\
\text { AlQadarif-Kosti }\end{array}$ \\
\hline $\begin{array}{l}\text { Ethiopia/ } \\
\text { Eritrea }\end{array}$ & $\begin{array}{l}\text { Yabelo } \\
\text { Asmara } \\
\text { Gambela }\end{array}$ & $\begin{array}{l}353 \\
349 \\
311\end{array}$ & $\begin{array}{c}\text { Awasa-Yabelo } \\
\text { Massawa-Asmara } \\
\text { Gambela-Malakal } \\
\text { Nekemte-Gambela } \\
\text { AddisAbaba-Nekemte }\end{array}$ \\
\hline Mali & Bamako & 807 & $\begin{array}{c}\text { Bamako-Kayes } \\
\text { Kayes-Tambacounda } \\
\text { Tambacounda-Kaolack } \\
\text { Kaolack-Dakar } \\
\text { Sikasso-Bamako } \\
\text { BoboDioulasso-Sikasso } \\
\text { Ouagadougou-BoboDioulasso } \\
\text { FadaNgourma-Ouagadougou } \\
\text { Niamey-FadaNgourma }\end{array}$ \\
\hline Ghana & Bolgatanga & 371 & $\begin{array}{c}\text { Ouagadougou-Bolgatanga } \\
\text { Bolgatanga-Tamale } \\
\text { Tamale-Kumasi }\end{array}$ \\
\hline D.R. Congo & $\begin{array}{l}\text { Kisangani } \\
\text { Lubumbashi }\end{array}$ & $\begin{array}{l}342 \\
303\end{array}$ & $\begin{array}{l}\text { Gulu-Bunia } \\
\text { Kitwe-Lubumbashi }\end{array}$ \\
\hline Guinea & Labe & 333 & Conakry-Labe \\
\hline Zambia & Chipata & 326 & Chipata-Lilongwe \\
\hline Cameroon & Yaounde & 302 & Douala-Yaounde \\
\hline
\end{tabular}




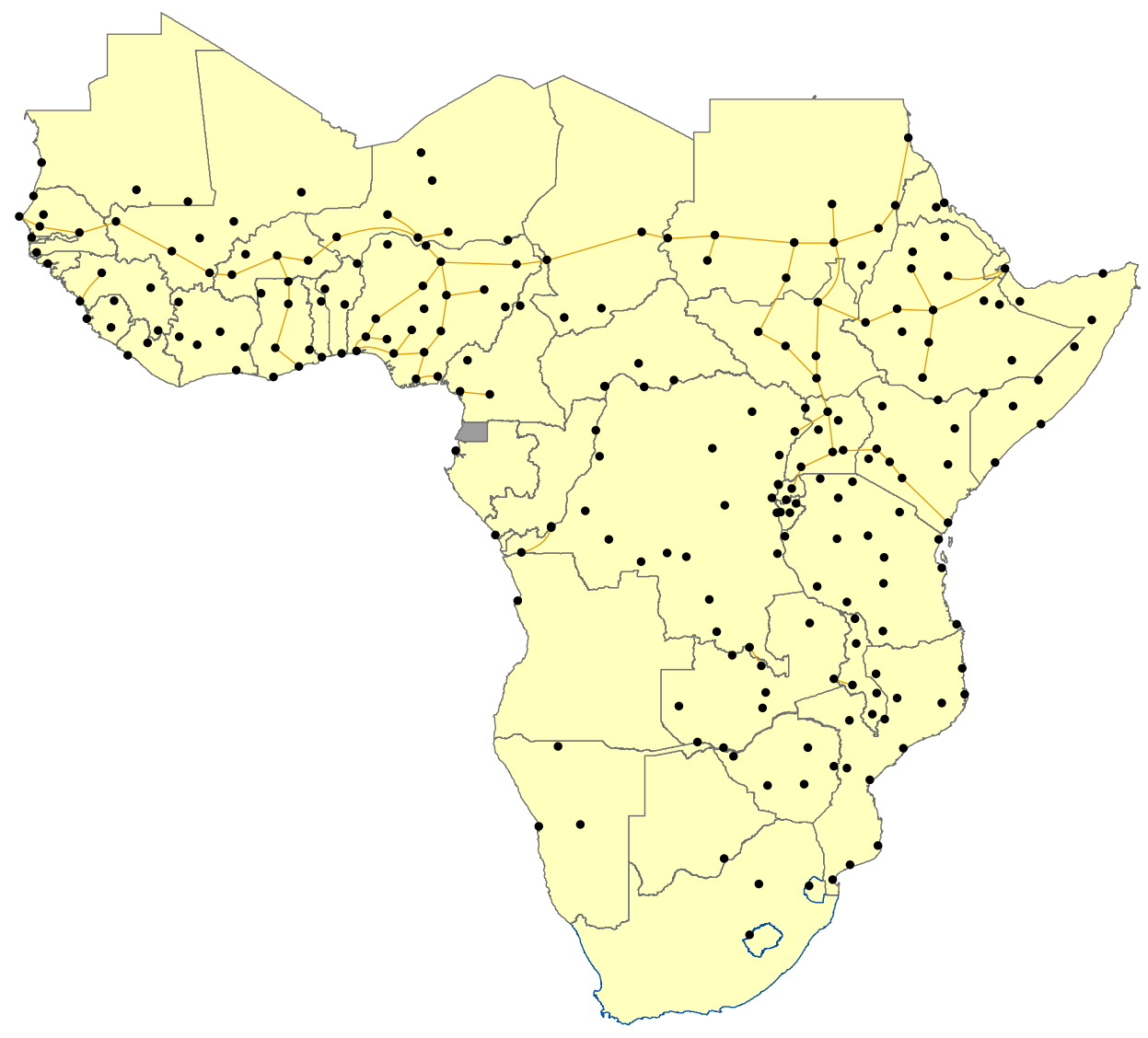

Figure A3: Map of 75 Targeted Links 
Table A18: Welfare Effects of Trade Cost Reduction for Single Link (Matadi-Kinshasa, D.R.C.)

\begin{tabular}{lccc}
\hline & Potential Gains & Achieved Gains & Percent Achieved \\
\hline Kinshasa & 876.4 & 831.2 & 94.8 \\
Bandundu & 93.3 & 32.6 & 34.9 \\
Gbadolite & 191.5 & 4.9 & 2.6 \\
Kisangani & 461.7 & 119.8 & 25.9 \\
Kikwit & 154 & 44.5 & 28.9 \\
Mbandaka & 207.3 & 100.4 & 48.4 \\
Tshikapa & 83.2 & 8.3 & 10.0 \\
Zongo & 153.4 & 50.8 & 33.1 \\
\hline Total & 2220.8 & 1192.5 & 53.7 \\
\hline
\end{tabular}


Table A19: 90 Links Corresponding to Proposed Trans-African Highways

\begin{tabular}{|c|c|c|}
\hline Highway & Affected Countries & Corresponding Links \\
\hline Cairo-Dakar & Mauritania / Senegal & Nouakchott-SaintLouis, SaintLouis-Dakar \\
\hline Algiers-Lagos & Niger / Nigeria & $\begin{array}{c}\text { Arlit-Agadez, Agadez-Zinder, } \\
\text { Zinder-Kano, Kano-Kaduna, } \\
\text { Kaduna-Ilorin, Ilorin-Ibadan, } \\
\text { Ibadan-Lagos }\end{array}$ \\
\hline Tripoli-Windhoek & Congo / D.R. Congo & Brazzaville-Kinshasa, Kinshasa-Luanda \\
\hline Cairo-Gaborone & $\begin{array}{l}\text { Sudan / Ethiopia / } \\
\text { Kenya / Tanzania / } \\
\text { Zambia / Zimbabwe / } \\
\text { Botswana }\end{array}$ & $\begin{array}{l}\text { Khartoum-AlQadarif, AlQadarif-Gondar, } \\
\text { Gondar-BahirDar, BahirDar-AddisAbaba, } \\
\text { AddisAbaba-Awasa, Awasa-Yabelo, } \\
\text { Yabelo-Moyale, Moyale-Wajir, } \\
\text { Wajir-Garissa, Garissa-Nairobi, } \\
\text { Nairobi-Arusha, Arusha-Dodoma, } \\
\text { Dodoma-Iringa, Iringa-Mbeya, } \\
\text { Mbeya-Kabwe, Kabwe-Lusaka, } \\
\text { Lusaka-Livingstone, Livingstone-Hwange, } \\
\text { Hwange-Bulawayo, Bulawayo-Johannesburg, } \\
\text { Johannesburg-Gaborone }\end{array}$ \\
\hline Dakar-N'Djamena & $\begin{array}{l}\text { Senegal / Mali / } \\
\text { Burkina Faso / Niger } \\
\text { Nigeria / Chad }\end{array}$ & $\begin{array}{l}\text { Dakar-Kaolack, Kaolack-Tambacounda, } \\
\text { Tambacounda-Kayes, Kayes-Bamako, } \\
\text { Bamako-Sikasso, Sikasso-BoboDioulasso } \\
\text { BoboDioulasso-Ouagadougou, } \\
\text { Ouagadougou-FadaNgourma, } \\
\text { FadaNgourma-Niamey, Niamey-Maradi } \\
\text { Maradi-Katsina, Katsina-Kano, } \\
\text { Kano-Maiduguri, Maiduguri-Ndjamena }\end{array}$ \\
\hline N'Djamena-Djibouti & $\begin{array}{l}\text { Chad / Sudan / } \\
\text { Ethiopia / Djibouti }\end{array}$ & $\begin{array}{c}\text { Ndjamena-Abeche, Abeche-ElGeneina, } \\
\text { ElGeneina-AlFashir, AlFashir-ElObeid, } \\
\text { ElObeid-Kosti, Kosti-AlQadarif, } \\
\text { Gondar-Dessie, BahirDar-Dessie, } \\
\text { Dessie-Djibouti }\end{array}$ \\
\hline Dakar-Lagos & $\begin{array}{l}\text { Senegal / Gambia / } \\
\text { Guinea Bissau / Guinea / } \\
\text { Sierra Leone / Liberia / } \\
\text { Cote d'Ivoire / Ghana / } \\
\text { Togo / Benin / } \\
\text { Nigeria }\end{array}$ & $\begin{array}{l}\text { Kaolack-Banjul, Banjul-Ziguinchor, } \\
\text { Ziguinchor-Bissau, Conakry-Freetown, } \\
\text { Freetown-Bo, Bo-Monrovia, } \\
\text { Monrovia-Gbarnga, Gbarnga-Man, } \\
\text { Man-Daloa, Daloa-Abidjan, } \\
\text { Abidjan-SekondiTakoradi, } \\
\text { SekondiTakoradi-Accra, Accra-Lome, } \\
\text { Lome-Cotonou, Cotonou-Lagos }\end{array}$ \\
\hline Lagos-Mombasa & $\begin{array}{l}\text { Nigeria / Cameroon / } \\
\text { D.R. Congo / Uganda / } \\
\text { Kenya }\end{array}$ & $\begin{array}{c}\text { Lagos-BeninCity, BeninCity-Enugu, } \\
\text { Enugu-Bamenda, Bamenda-Yaounde, } \\
\text { Kisangani-Bunia, Bunia-Butembo, } \\
\text { Butembo-Mbarara, Mbarara-Kampala, } \\
\text { Kampala-Jinja, Jinja-Eldoret, } \\
\text { Eldoret-Nakuru, Nakuru-Nairobi, } \\
\text { Nairobi-Mombasa }\end{array}$ \\
\hline Beira-Lobito & $\begin{array}{l}\text { Mozambique / Zimbabwe / } \\
\text { Zambia / D.R. Congo }\end{array}$ & $\begin{array}{l}\text { Beira-Chimoio, Chimoio-Mutare, } \\
\text { Mutare-Harare, Harare-Lusaka, } \\
\text { Kabwe-Kitwe, Kitwe-Lubumbashi } \\
\text { Lubumbashi-Kolwezi }\end{array}$ \\
\hline
\end{tabular}

